\title{
Negotiating a Quantum Computation Network: \\ Mechanics, Machines, Mindsets
}

\author{
by
}

\author{
Derek Noon
}

\begin{abstract}
A thesis submitted to the Faculty of Graduate and Postdoctoral Affairs in partial fulfillment of the requirements for the degree of

Doctor of Philosophy

in
\end{abstract}

Communication

Carleton University

Ottawa, Ontario

(C) 2016, Derek Noon 


\section{Abstract}

This dissertation describes the origins, development, and distribution of quantum computing from a socio-technical perspective. It depicts quantum computing as a result of the negotiations of heterogeneous actors using the concepts of ANT and socio-technical analyses of computing and infrastructure more generally. It draws on two years of participant observation and interviews with the hardware and software companies that developed, sold, and distributed both machines and a mindset for a new approach to computing: adiabatic quantum computation (AQC). It illustrates how a novel form of computation and software writing was developed by challenging and recoding the usual distinctions between digital and analogue computing, and discusses how the myriad controversies and failures attending quantum computing were resolved provisionally through a series of human and non-human negotiations. These negotiations disrupted, scrambled, and reconstituted what we usually understand as hardware, software, and mindset, and permitted a 'disruptive' technology to gain common acceptance in several high profile scientific, governmental, and financial institutions. It is the relationalities established across these diverse processes that constitute quantum computing, and consequences of this account of computation are considered in the context of digital media theory, industrial histories of computing, and socio-technical theories of technological innovation. 


\section{Acknowledgements}

Many sources of support helped me through the PhD program.

I'm grateful to Mitacs for its financial support of this research, and for providing me such good STEM peers/research subjects.

This project could not have taken the shape it did without the help and participation from the many people at $1 \mathrm{QBit}$, who let me into their space and brains, gracefully answering endless questions. Thank you especially to Andrew Fursman, for giving me the opportunity to spend time in such a fascinating research location.

Many Carleton faculty members have given me opportunities to research, lecture, and teach, while providing the encouragement that helped fuel this work. My early theoretical framework benefitted from the guidance of Dr. Melissa Aronczyk who supervised my comprehensive exam and helped me reflect on my writing and research goals. Dr. John Shiga gave great advice for approaching Actor-Network Theory from a media studies perspective and where to begin reading. Dr. Sandra Robinson has been a great example of how to write about highly technical subjects and her support through the last mile of this dissertation is deeply appreciated. 
I am very grateful to my committee for participating in this project. Thanks you Dr. Carols Novas for taking an interest in my work and supporting its development. Dr. Brian Greenspan and Dr. Bart Simon were invigorating external examiners who have each given me a great deal to consider for future directions in my research. Dr. Sheryl Hamilton's questions/comments always manage to get right to the core of a subject, and have helped me appreciate how to give better feedback myself. Her support throughout each step of the program and dissertation has been invaluable.

I want to sincerely thank Dr. Chris Russill for supporting this dissertation since its earliest stages. It made a massive difference to have a mentor so engaged in the questions and ideas at play in my research, able to understand what my clumsy first drafts were getting at, and who encouraged me to showcase my work. His dealing with the administration of this research has also been significant and deserving of praise.

I was lucky to be grouped with a great cohort of PhDs and MAs that made the program's courses and comprehensive exams enjoyable challenges, too many to mention. Thanks are particularly owed to Bill for his advice and example, Laura for ongoing encouragement/threats, and Kolar for her insight into sociology and presence during the difficult middle years of this project. 
Thank you to my family for their support, and for trying not to ask when I will be done too often.

Thank you especially to Sonja, for her patience listening to me ramble about quantum nonsense, her proofreading skills, and most of all her company.

Lastly, thanks to all the nonhumans who made this project possible. 


\section{Table of Contents}

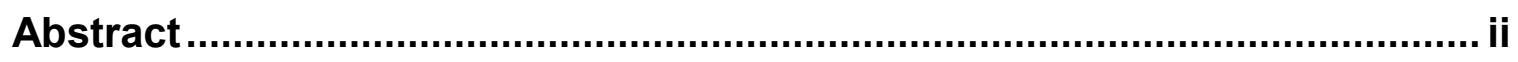

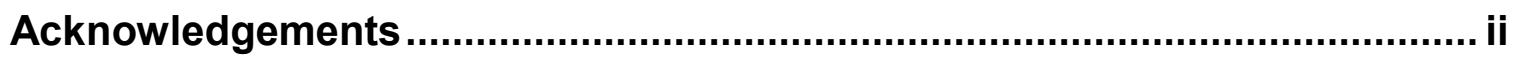

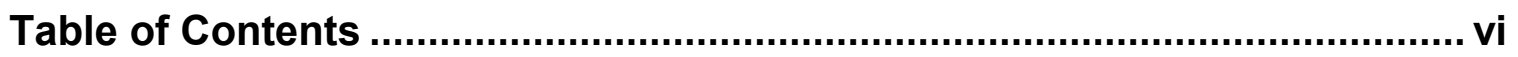

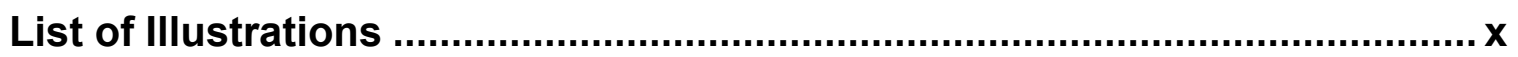

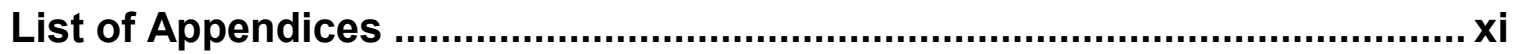

1 Chapter: Determining the Quantum State: Opening Negotiations for

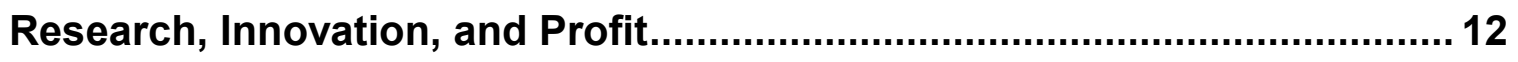

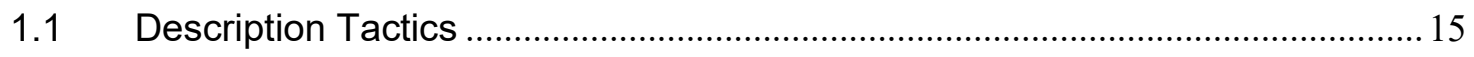

1.2 Disrupting Narratives of Disruption ............................................................... 18

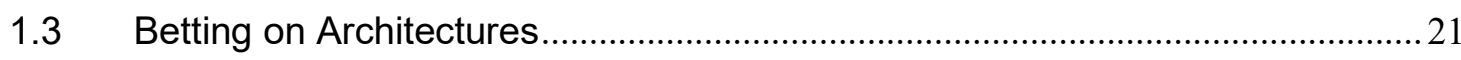

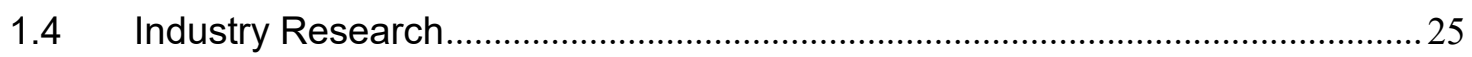

1.5 Commercializing Quantum Computing ...................................................................26

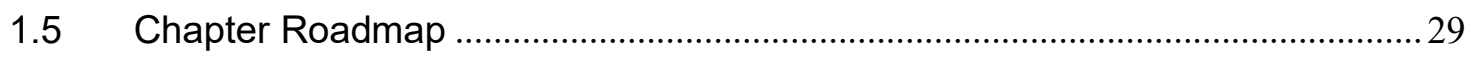

2 Chapter: Socio-technical Approaches to Technology and Computation33

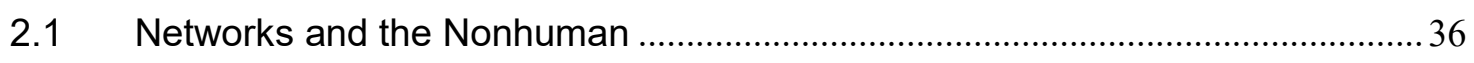

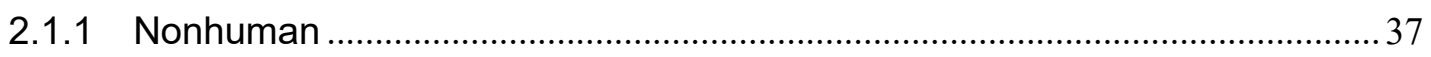

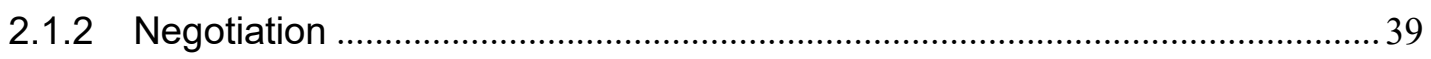

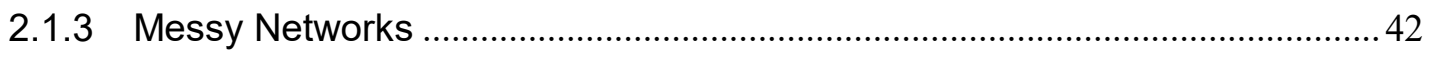

$2.2 \quad$ ANT and Critical Computation ..........................................................................

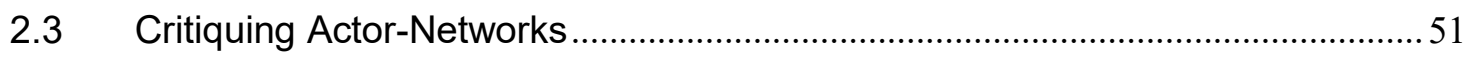

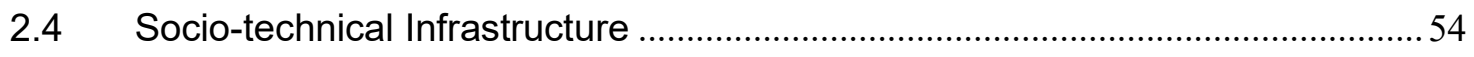

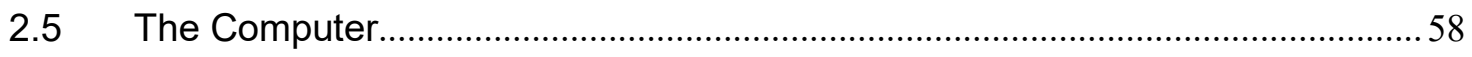

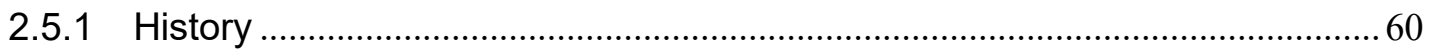


2.5.2 Media Theory

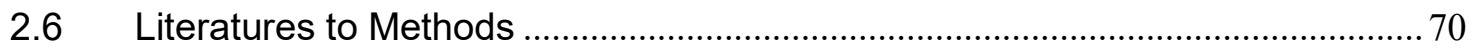

3 Chapter: Expanding Translation as a Method for Techno-Messes ......... 71

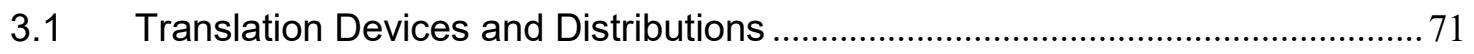

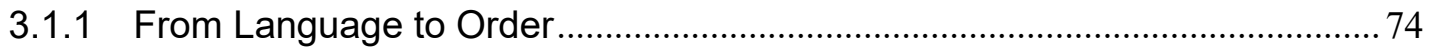

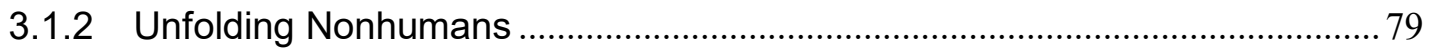

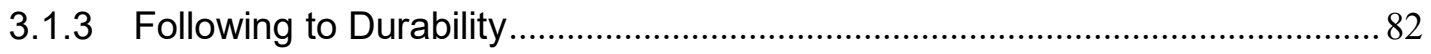

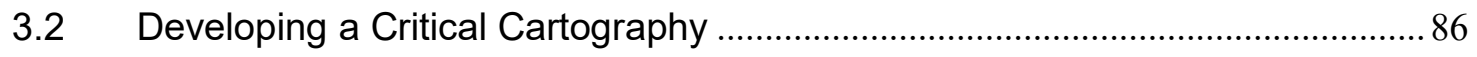

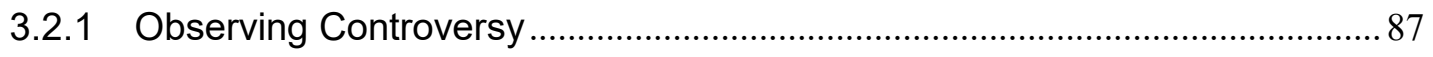

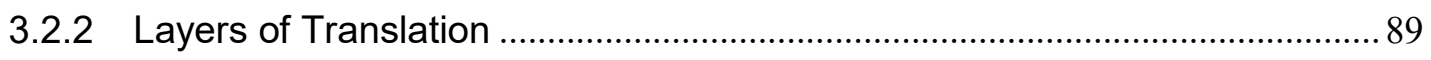

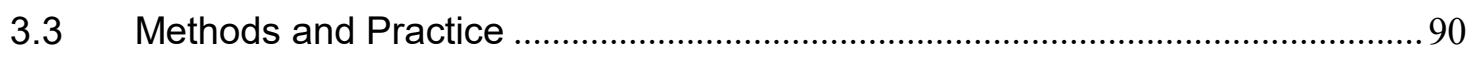

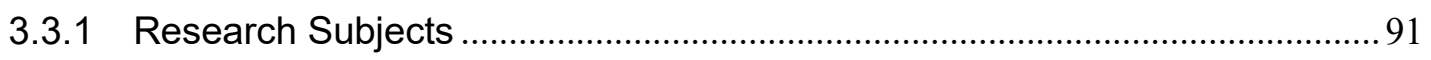

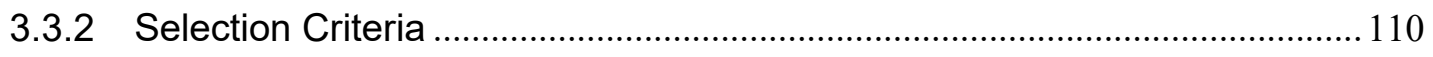

4 Chapter: Gathering Distinctions for the Production of Qubits .............. 114

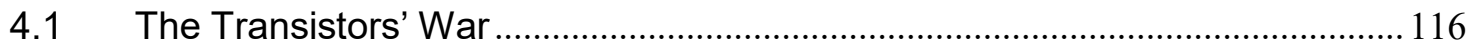

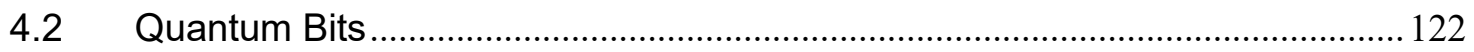

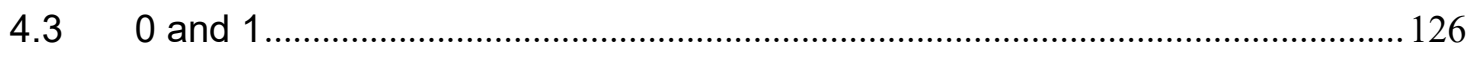

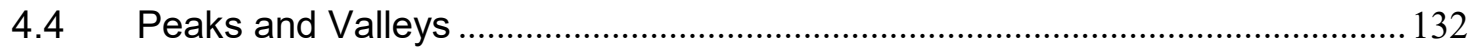

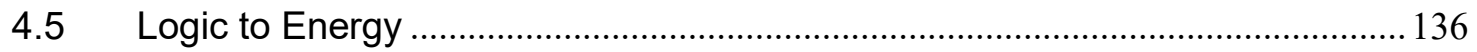

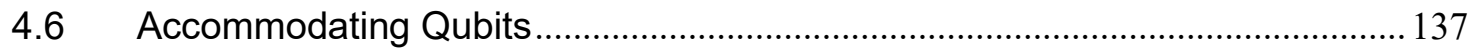

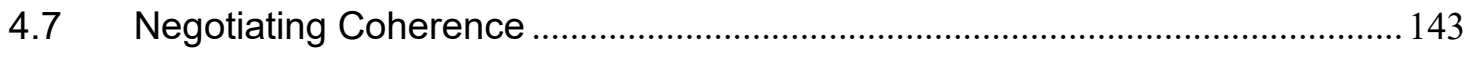

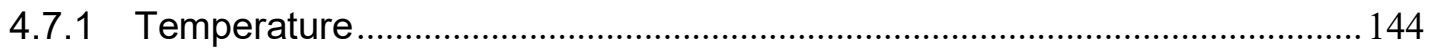

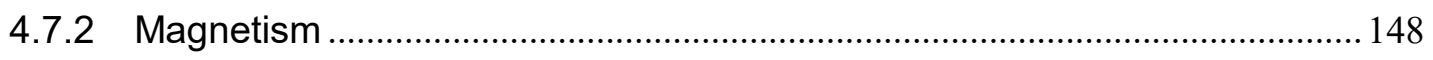

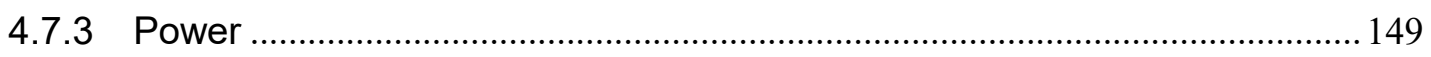

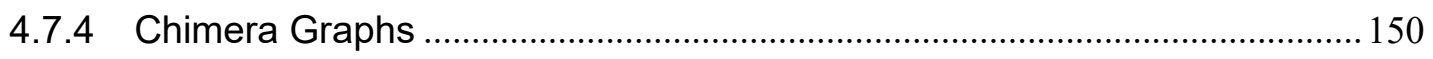




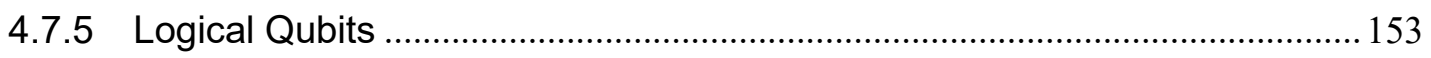

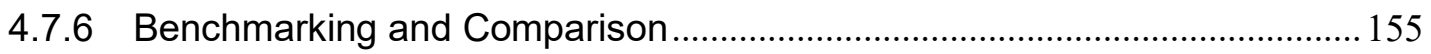

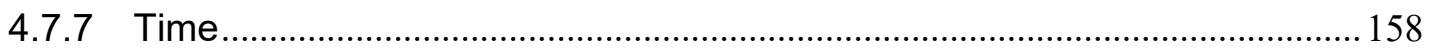

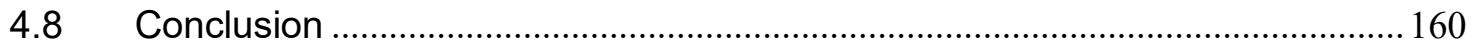

\section{Chapter: The Portfolio and the Processor: Negotiating Problematics to} Enable Quantum-Hybrid Software .......................................................... 162

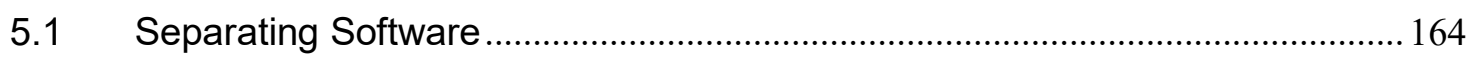

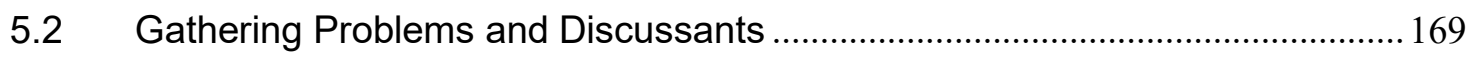

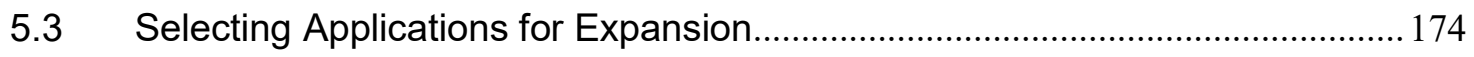

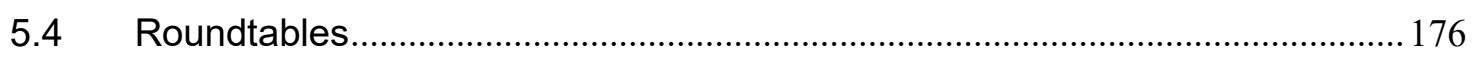

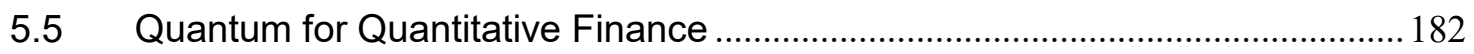

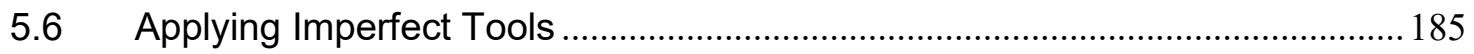

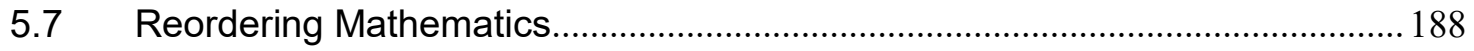

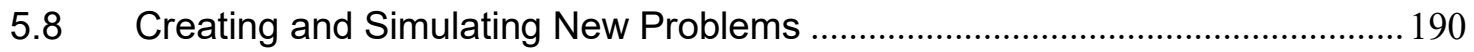

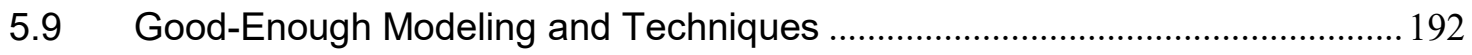

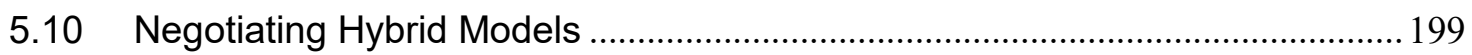

5.11 Expectation Versus Emergent Solutions ......................................................... 201

6 Chapter: From the Quantum Computer to Quantum Ready: Distributing a Mindset in an Uncertain Actor-Network ................................................... 205

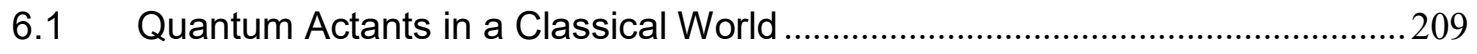

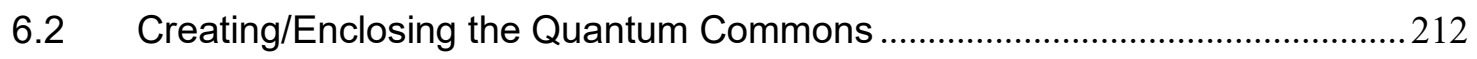

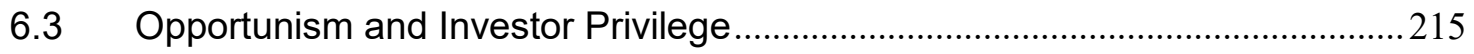

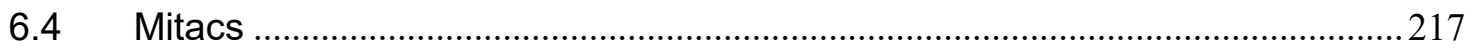

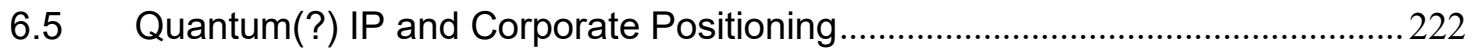

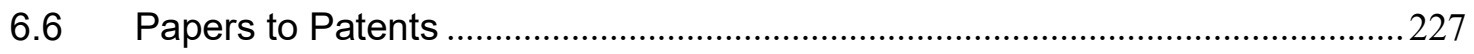

6.7 Hocking Applications-Driven Development for Credibility and Access ...........233 


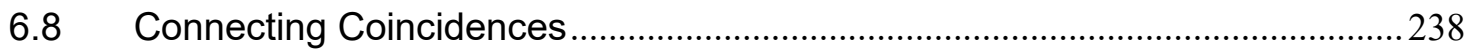

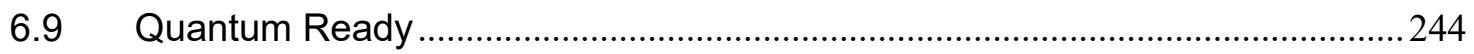

7 Chapter: Conclusion: Computing in Uncertain Times ......................... 248

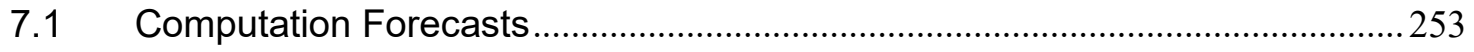

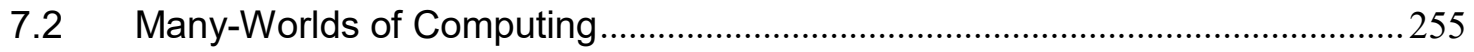

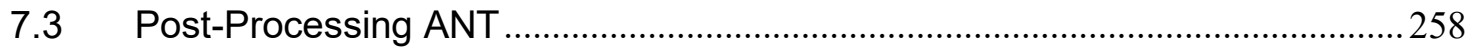

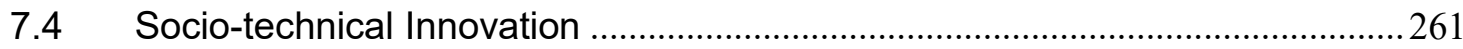

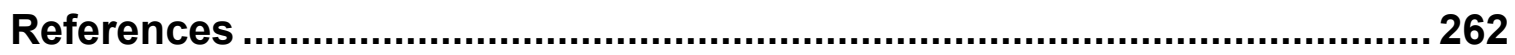




\section{List of Illustrations}

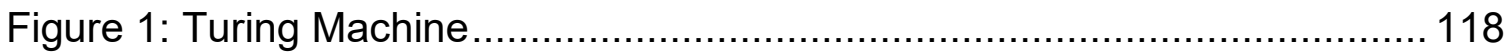

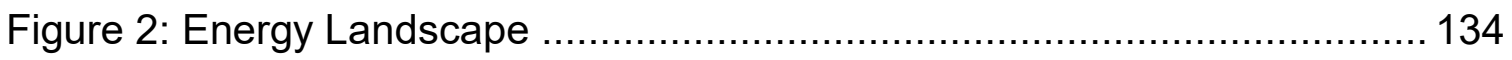

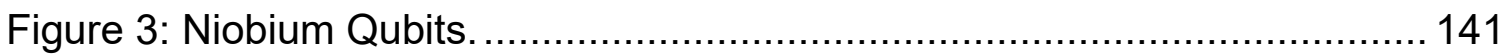

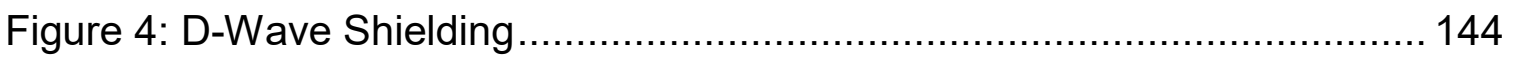

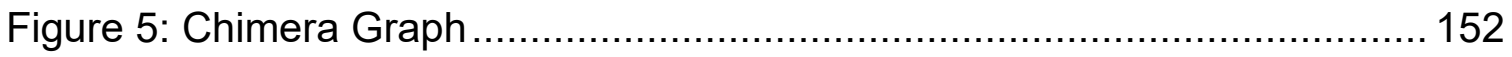

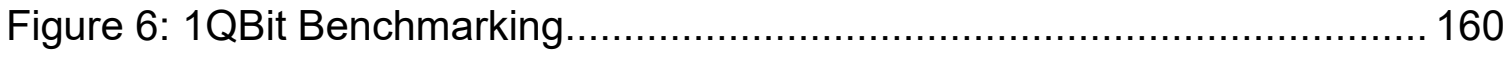

Figure 7: Digital Computer Layer Visualization ............................................ 168

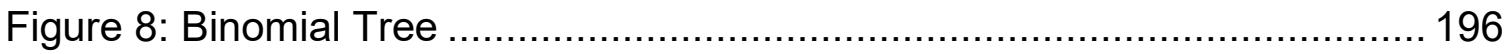




\section{List of Appendices}

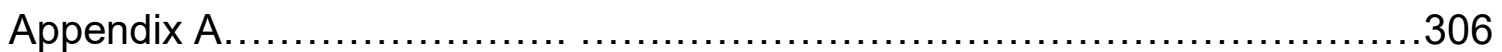

A.1 Initial 1QBit Core Readings...........................................304

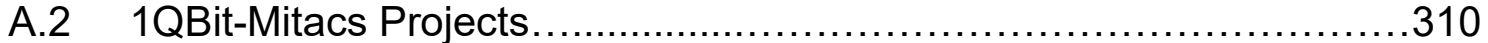




\section{Chapter: Determining the Quantum State: Opening}

\section{Negotiations for Research, Innovation, and Profit}

Lining the walls of a small office building in Burnaby, $\mathrm{BC}$, are a series of black boxes the size of a cubicle. Their sides are covered in glowing lights, and they periodically hum and hiss. Each one is colder than anywhere else in the universe, costs 15 million dollars, and nobody is certain what they can do.

Their inventors argue that these boxes contain a futuristic method of computation that can solve currently intractable problems. They have been proclaimed as nothing less than the first true paradigm shift in computation since binary, challenging the basic concepts that underlie computers and allow them to do work. To the companies that work with them, these boxes are a "pillar of computational progress", ${ }^{1}$ whereas other developers see them as part of the "quantum risk" ${ }^{2}$ that threatens the secure operations of all the digital computers in use today. Many are watching closely from the sidelines of these developments. Investors debate how and where to direct funding, while governments promote their support to signal forward looking agendas. In many physics departments they are called snake-oil hoaxes, experimental dead-ends, or conversely, brilliant work. Popular media, from Time magazine to personal blogs, describe them as the first wave of an incoming tide of futuristic computers

\footnotetext{
${ }^{1}$ http://1qbit.com/about/

${ }^{2}$ http://www.etsi.org/news-events/events/1072-ws-on-quantumsafe-2016
} 
with unheard of computational abilities. Whether the forecast is positive or negative, quantum computers are a just out-of-reach reality; already arrived, but as a preview of a computational future, with which we can contrast the current moment and its digital limits.

These strange glowing boxes are a branch of the ongoing effort to develop new and more powerful methods of computation that has routinely driven both the commercial computing sector and the disciplines of computer science and engineering. This effort unfolds in three mainly separate spheres: hardware, software, and institutions. These distinctions have been normalized by the computing industry, through intentional market segmentation and labour specialization, as well as anti-trust actions. Innovations in hardware since the mid twentieth century have increased the speed and decreased the size of computer components. New programming languages have often shaped software development, as has the development of algorithms that use increasingly 'big' data resources to evolve themselves. The institutions that oversee and perform these advancements have shifted between government, corporate, and academic organizations, driving resources and research priorities to and from new areas.

The new branch represented by the development of quantum computers actually undermines many of the assumptions and distinctions that structure these spheres of computational development. It challenges how we think about computers by creating uncertainty in almost all aspects of their operation: logical, 
material, environmental, institutional, legal, cultural and so on. These new computers present a radical break from the methods, media, and mindset used in the past for computation, as well as predictions about how those methods were to be improved upon, to the point we are forced to ask: do we know what computation is? The 'digital' has structured how we understand computation for half a century and developed both technical and more nebulous connotations for the many objects and activities created by computation. Quantum computation recasts the digital as merely one example of what is now called 'classical' computing, and as a result our assumptions about computation become more diverse and less certain.

I first found myself in the same room as the glowing boxes in June of 2013. Like many on that factory floor tour, I took selfies inside and out of their massive cages, and picked up the wafers of processors to squint at their tiny circuitry, replicated thousands of times in my hand. In one room in the factory, workers similarly squinted through magnifying glasses to carefully lace together the wiring that would connect and cool those circuits. It took much longer than the trip to British Columbia to later grasp what I had been holding, and this was only possible by spending much more time squinting at all sorts of written and visual representations of those devices, and the people working with them. Like me, those researchers and developers were trying to figure out for themselves what these devices were doing, and what else they could be made to do. I watched this unfolding negotiation and found the deliberations between the people and 
the seemingly impenetrable boxes as intriguing as the exotic circuitry. Studying one quickly meant studying the other. As more negotiation tactics were deployed and the number of people and technologies involved grew, they shaped a distinct network for computation. This dissertation traces the negotiations that comprise this network, and how a computer and computational mindset shape one another.

\subsection{Description Tactics}

The development of quantum computing is typically described as a paradigm shift in computation by its practitioners in both academia and private industry. Those taking part in its development consider it to be the first real fundamental change in how computers work, on a scale completely different than the many changes in performance and form that have occurred in the myriad iterations of digital computers, from vacuum tubes to cloud servers. By these accounts, quantum is the major moment of disruption in the computing industry that had been established with digital technology. This depiction of technological disruption, paradigm shift, and the implied accompanying industry-revolution, has been a useful framework for those attracting attention and capital to the projects at the forefront of quantum computing research.

I adopt an alternative view to the disruption narrative by articulating quantum computing using a socio-technical framework. This dissertation develops an ontology of quantum computing, examining how a new mode of information 
processing is coming into being. This helps appreciate the connections to, as well as the breaks from, other kinds of computation such as the digital. Instead of assuming digital and quantum as a past and future state of computing, they reflect differently assembled networks of computational resources formed around separate problems and materials. Building a new socio-technical network for quantum computing involves disentangling many of the humans and nonhumans from their old sets of ties, and reframing these in a new set of relationships. Due to this task of reordering and connecting the many diverse players in a computation system, considering quantum computing brings forth the full breadth of industrial engineering and business practices at play in shaping computation. These challenges to the norms of classical computing suggest computation as an open, unstable, and negotiated terrain. This indicates that studying computation requires an approach able to conceptualize the diverse forces that meet at the nexus of a new machine, and can characterize the scale, instability, and messy entanglements involved.

Actor Network-Theory (ANT) is a good departure point to ask how a new approach to information processing is brought into existence given the complicated socio-technical landscape presented by quantum computing. ANT's approach to examining contentious, unstable technical objects and contexts, is well suited for the heterogeneous forces or agencies at play in shaping quantum computing. Its approach of tracing such varied relationships, the networks they create, and the patterns of action they enable, can work to conceive, describe, and analyze the indeterminate processes at work. With few exceptions (Latour 
2010), there has been little application of ANT to computers or suggestions of how to study computation using this perspective. This study suggests that the relations that form computation can be approached through ANT, and it is well suited to frame the recent developments of quantum computing and what they can tell us about the negotiations and delegations that allow a new technology to emerge. To do this, I borrow from early ANT writing on translation, as well as the post-ANT reflections of its founders, to develop a socio-technical analysis using concepts of ontological gathering, the agency of problematics, and the development of computational mindsets. These help explore the unfolding history of quantum computation and tap computation as a role model for studying complex, messy, uncertain, and unsettled technologies.

Similar to many ANT studies, this project began with a focus on a controversial new technology that involves tracing the agencies of new nonhuman actors. However, unlike many of the examples discussed in Chapter 2, I do not seek to explain a singular moment of failure of a new network or highlight its established durability. Instead, I try to attune ANT to the process of technical construction in a state of deep uncertainty and the hybridizations that result. In the following chapters, I deploy and develop the ANT concept of translation, with its radical approach to relationality, to understand the processes of establishing the new connections (i.e. material, syntactical, legal, etc.) necessary to bring quantum computing into the world. This project also highlights work that is usually behind closed doors of private research. ANT is useful here for showing how this work 
has many kinds of influences or actors that change plans, possibilities, and outcomes as they interact to form a technological development.

\subsection{Disrupting Narratives of Disruption}

This dissertation does not debunk or disprove the grandiose claims made about the capabilities of quantum computing to revolutionize computation. It does aim to sidestep the entrenched optimism at the core of the 'disruptive' narrative so often at the fingertips of its developers. Chapters 4-6 depict the development of quantum computing by tracing how the construction of computer systems, problematics, and mindsets, define its actor-network. When interviewed, many of the researchers working on these problems articulated an incredibly optimistic view of technological development. In part, this was tied to the context of quantum computing arising as a solution for problems in optimization studies. As one researcher put it, "In optimization, you are making something better. When you make lots of somethings better...it adds up. Society improves". However, they were quick to point out the very few applications and users that were likely to benefit from a process becoming more 'optimal'. Similarly, nearly all those interviewed repeatedly stressed the significance of the novelty of shifting computational paradigms. Yet, over time and in follow up discussions, the same researchers were eager to make sure I did not confuse what they were doing for textbook definitions of quantum computing. Instead, they stressed the hybrid nature of the technology they were in the process of creating. Their reflections on technology, and their practices and description of specific technologies, were often somewhat disconnected. The socio-technical approach taken in the 
following chapters does not try to close that gap. The way that different descriptions and actions layer, combine, mutate, and reinforce one another is not a conflict but a working construction strategy.

If quantum computing is a hybrid technology that diverges from textbook ideals, as discussed below, it is also a hybrid mindset, a strange mixture of optimism and anxiety, of hyperprecision and mysticism, of basic science and profound confusion, of uncertainty and confidence, and this too fails to conform with the usual narratives of disruption, paradigm shift, or progress through technology. Much of this is rooted in its foundation of quantum mechanics, which leads to many of the seeming contradictions and complexities involved in this method of computation.

Quantum Mechanics is a mathematical framework used to make predictions about physical phenomena. It is usually associated with the very small, such as behaviours at the atomic or subatomic scale. At these scales, the predictions offered by quantum mechanics differ substantially from what is typically referred to as classical or Newtonian physics. At such small scales, classical physics ceases to produce accurate predictions, while quantum mechanics has proven to be extremely accurate. In order to be so accurate however, quantum mechanics assumes a worldview that seems to conflict with humans' everyday perception of reality. Commonsense assumptions, such as that an object can only be in one state or location at a time, are upended. 
The physicists responsible for breakthroughs in quantum mechanics have been deeply discomforted by these implications. Quantum Mechanics has been famously "unpalatable" for many scientists and theorists (Nielsen and Chuang 2). Well-known examples include Einstein's objections to the quantum worldview, Bohr's comment that anyone who knew the theory should be "shocked", and the popular thought experiment of Schrodinger's cat, which originally highlighted the ridiculousness of quantum mechanical assumptions (Nielsen and Chuang 2011, $\mathrm{Xi}$, Emerson 2011). A certain amount of mysticism has also accompanied the field. The popular quotation often incorrectly attributed to Feynman, "if you think you understand quantum mechanics, you don't understand quantum mechanics", reflects an earlier decision among many physicists who follow the so-called Copenhagen Interpretation. This approach simply accepts the unintuitive but accurate results of quantum mechanics with the wonderful summary: "Just shut up and calculate!" (Mermin 2004). The strange assumptions of quantum mechanics lead to equally strange suggestions for quantum computers. Some of their developers claim they work by tapping into other universes, as part of the many-worlds theory. ${ }^{3}$ Another widely recognized concept of quantum mechanics, the uncertainty principle, outlines how quantum states are always altered through interactions with classical objects. This means that the interior of those black boxes is impossible to observe while they are computing. This creates room for uncertainty about exactly how they accomplish their calculations. Many of their developers, however, profess not to care how it works, and focus on the process

\footnotetext{
${ }^{3} \mathrm{D}$-Wave founder Geordie Rose is well known for making this suggestion about AQC, however engineers at the company prefer to avoid these metaphysics.
} 
of engineering rather than theorizing quantum computers. Yet the mindset that permits this theoretical agnosticism among physicists working on quantum mechanics, is difficult to sustain for those trying to get quantum computers to calculate new, bigger, harder problems. As I discuss below, they must 'open up' not 'shut up' and connect these hidden, uncertain, interiors to the outside nonquantum world and its computational concerns. Chapter 4 describes this transition from research in quantum mechanics to the proposal and construction of quantum computers.

\subsection{Betting on Architectures}

Even without a broad concern with the inner-workings of reality, confusion and uncertainty remain. The engineering of quantum computers in many aspects upends the algorithmic concepts at the heart of computer science that date to at least Hammurabi (Nielsen and Chuang 2011, 4). Numerous computational devices have been theorized or built to run increasingly sophisticated algorithms at different points in history, some of which resemble the modern computer. Often cited examples include Babbage's analytic engine in the 1800s, the Marchant calculator in the early 1900s, and even the ancient Antikythera mechanism, circa 200 BC (O’Regan 2008, Ceruzzi 2012). The many differences between these devices are relatively flattened by the changes in algorithmic thinking that become assumable when working with quantum mechanical machines. The aura of mystery, hyper-precision, and scientific advancement that has latched onto the notion of quantum, has helped increase the excitement 
around the prospect of a quantum computer in popular culture as well as for venture capitalists.

Quantum systems offer a new kind of computing that is not well understood in communications or the broader public, even as it becomes a more recognized term. Quantum computers have been a common feature of science fiction literature. However, during the course of my research it also became common to find coverage about quantum computers in magazines, news websites, and popular blogs. Before my research period, this would have been highly unusual outside of dedicated science publications. An important factor that complicates an outsider keeping track of the development of quantum computers, is the diversity of projects being called quantum computation. Many different materials are being explored as potential components of a quantum computer, and each have their own significant milestones being reported on (e.g. Noguchi et al. 2011, Taminau et al. 2014). However, these many projects fall into two distinct approaches to quantum computation. The most common approach is the attempt to build a universal quantum computer that, in many ways, resembles the structure of digital computers, including the use of gates to sort information, and the flexibility to model any problem, to be what is understood as a "general purpose" computer. As Chapter 4 will discuss in more detail, this is an incredibly challenging engineering approach that requires significant long-term basic research to be accomplished. The vast majority of research being done for quantum computers falls in this category, whether by academic departments or 
corporate labs. It is a vision pursued by teams at Oxford University, Microsoft, and many other elite institutions. Though each of these groups attempt to do so with different designs and embodiments. The dream of this universal quantum computer has a long established lineage in physics departments (see Chapter 4), and when the possibilities of quantum computing are described in popular accounts, it tends to be with this model and its implications in mind.

Animating my investigation has been the surprising propagation and early success of another approach, called Adiabatic Quantum Computation (AQC). As will be seen in the examples found in the following chapters, AQC has drawbacks compared to the ideal of general purpose quantum computing. However, despite its limitations, it has proven easier to engineer in the short-term. These different visions of how quantum computing should be done has created controversy and an uncertainty over how and when an advantageous use of quantum computing will be put into practice beyond experiments and prototype demonstrations. These rival computer architectures compete for investment and support from government, corporate, and academic actors, but as will be seen, also connect with one another as part of a growing mindset around quantum-based computation. This dissertation focuses heavily on AQC, though necessarily brings it into contrast with the general-purpose model as they increasingly define one another in various ways: as practical/limited, theoretical/applied, corporate/academic, distant/current, etc. In Canada, these approaches have been pursued in different geographies. The basic research behind a general purpose architecture is strongly focused in Ontario, particularly through the 
University of Waterloo and its partner organizations. AQC has been pursued on the west coast, most actively by companies in Vancouver that have strong ties to the University of British Columbia and Simon Fraser University.

This project hopes to increase the literacy around the spectrum of quantum computing, to appreciate the diversity of actors and possibilities that are frequently collapsed under the quantum umbrella. This can help those in media and communication studies reconsider their assessments of computation that assumed solely a digital paradigm, and to bring to light some of the issues that would arise in too simply contrasting the digital and quantum. This dissertation will not argue that we now need a 'quantum humanities' in order to update the field, but does seek to shake our assumptions about computation that are tied explicitly to the digital. Previous personal experience with digital computers, which has guided much writing about software, computers, and computation in recent studies (Fuller 2008), is ill-equipped to approach these foundational changes to computation posited by quantum computing. In response, my research offers a thick description of how quantum computing has assembled today as a connection of organizations, people, knowledges, and materials. I describe how a novel form of computation comes into being, and show how the acts required to develop this computation can inform a view of technological innovation as a process of negotiation. Quantum computing has moved from being a speculation made by a small group of theoretical physicists, to a part of the speculations of investors. I hope this research will help bring its discussion into the realm of communication and media studies as well. 
I use this thick description to show how the shape of such an emerging network is impacted by very different types of actors with different kinds of agencies, as they attempt to find methods to work together. This raises the questions of to what extent technology is planned, and to what extent it is the result of an encounter with material agencies and constraints. Given the omnipresent role of computation in the human environment, this negotiation of agencies around new computational possibilities results in significant inequalities for those who have access to them and those who do not. In the midst of development, however, what those inequalities will be is as couched in uncertainty as the quantum phenomena at the core of this case study. As quantum computing worldviews compete with one another for compliance with physical phenomena, their proponents need to grow networks to support and enact them while addressing doubts about the technology's prospects. How the materials called upon to enact quantum computing thus far have contradicted its early conceptualization has made the AQC approach controversial, as Chapter 6 explores. This affects the routes it can take to develop and what it must do to gain support while it matures.

\subsection{Industry Research}

This project focuses on technological change and innovation at its earliest stages. Before a technology circulates among the public or a clientele, I want to see what forces, motivations, agencies or agendas shape it and are inscribed into its embodiment. Discussions of the relationship of technological development, industry, and public discourse within media and communication 
studies often focus on a limited sample of media texts and frequently fail to detail the complex industry/state/academic relationships that attend any form of capitalintensive development. Research into the "technological" or "digital" sublime, for example, is important as a means of ideological critique and for demonstrating how industry promotion permeates public discourse. Yet, it doesn't allow us to see how industry/state/academic configurations produce computational networks, or to assess if such promotional discourse is simply false or actually helps steer the development of a technology. Similarly, Turner's (2006) historical account lacks a deep examination of industry, preferring to focus on academic and media entrepreneurs. Kittler also tends to shy away from in-depth accounts of the industrial development of complex computer-media interactions, as does the body of work focused on computer users and programmers (e.g. Wark 2004, Fuller 2008, Galloway 2012, Coleman 2013). This dissertation offers the first fulllength study of how a quantum computer, a novel new technology, comes into being as a configuration of state-corporate-academic actors. In this context, I examine how an emerging technology is resolved from the many possibilities of its configuration. To do this, I focus my research on the private sector hardware and software companies developing, selling, and promoting AQC technology. The peculiarities of this approach to computing arise from the strategies and applications for this corporate context.

\subsection{Commercializing Quantum Computing}

A number of experiments have produced basic quantum computers along the 
lines physicists have elaborated since the 1980s. Though these have produced impressive experimental achievements, a full general purpose computer that could run a meaningful program is not expected to be developed along these lines for several decades. Considering the difficulty in these incremental steps, it was to the surprise and skepticism of the physics community that D-Wave Systems, a relatively unknown company located near Vancouver, BC, claimed to have a "128-qubit" (see Chapter 4) computer for sale in 2011. That year, Lockheed Martin purchased the first commercial model at a cost of $\$ 10$ million. D-Wave Systems had earlier claimed a working 16-qubit system in 2007 , and immediately began to receive positive media attention for promises of a quickly scaling quantum technology that could soon be put to commercial use. Outlets such as the Economist (“Orion's Belter", Feb 15, 2007) claimed, "The world's first practical quantum computer is unveiled". By 2013, D-Wave produced 512-qubit models, selling them to a Google-NASA collaboration, and Lockheed Martin and others for $\$ 15$ million each. D-Wave currently claims to have a 1024-qubit model in testing. The IEEE Spectrum's 2010 report “D-Wave Does Not Quantum Compute" reflected a strongly skeptical response that began appearing across tech blogs and mainstream outlets which countered the enthusiastic corporate/media narrative throughout (e.g. Economist, Forbes, Time). How was D-Wave able to make such claims? The main reason, which escaped the majority of early press coverage and frustrated academic observers, was that D-Wave was attempting the AQC model of quantum computation. The nuances of this model are explored in detail in Chapter 4. 
Before D-Wave, "what is quantum computing?" had an evolving but less disputed answer, relevant to a small group of people in physics departments. After DWave, there are a plurality of answers and many new questions. Now that an apparently quantum device exists off the pages of physics papers, new and more pragmatic concerns are created. What can the machine be made to do? Could it solve problems any faster? Who should care and how will they be made to? DWave and its hardware are discussed throughout this dissertation, but primarily in the context of the company working on applications for these computers, $1 \mathrm{QB}$ Information Technologies (1QBit). Many of the changes catalysed by D-Wave, are trying to be settled into a new state, or program of action, for computation by $1 \mathrm{QBit}$. The details of my placement at $1 \mathrm{QBit}$ through the Mitacs internship program are outlined in Chapter 3. The majority of this dissertation describes the research being done on AQC at $1 \mathrm{QBit}$, positing it as an example of ANT's notion of an obligatory passage point, and central to understanding the formation of the current quantum computing industry (see Chapter 6). As will be expanded on in the following chapters, 1QBit appears as the software development partner of a hardware company. In many ways this is accurate, as it writes code, programs, and creates software applications. However, its work reaches well outside of the established norms of software development. 1QBit's slogan, "Redefine Intractable", hints at the broad translation work the company attempts. 


\subsection{Chapter Roadmap}

Chapter 2 notes the dearth of ANT studies on computation, and surveys ANT literature for the concepts used there to approach socio-technical subjects, arguing they have much to offer media and communications scholars examining the production of computer technology. This is contextualized with approaches to studying computers and computation in communications, which offer many useful frameworks that can inform an ANT study in this area.

The third chapter develops the ANT conception of agency, and its related notion of technological development, in terms of the concept of translation. Here I examine the uses of translation and its role as a methodology in the study of socio-technical systems. The chapter considers translation first as a guiding principle in enacting an ANT study, before exploring the ways this has been interpreted to guide the choice of specific objects of study and research methods. I then outline the ways in which I used this approach to engage with the human and nonhuman actors found in the quantum computing actor-network I examine.

The following three chapters feature key translations in the unfolding of the quantum computing actor-network. These translations depict entry points into what would usually be easily understood as the separate spheres of computational work: hardware, software, and institutional dynamics. What these translations make evident, is that in quantum computing, these distinctions have not settled down or ossified into separate spheres that let us distinguish sharply between what counts as hardware, software, or institution. The work we would 
expect to find in each is blurred and hybridized with others, around what Latour would term "monstrous" hybrid objects and acts, many with suitably monstrous names from volcanoes and mythical beasts.

In describing these moments of actors becoming a network for quantum computing, I emphasize the blurry, messy, shape-shifting process of developing computation. In turn, each of these indicate a transitional moment for quantum computing, as it compromises and negotiates its way from existing as a theoretical concept or vision alone, into a distributed, material, embodied system performing technical and social work in specific contexts. These examples highlight the complex dynamic of these hybrid objects arising in a non-hybridized digital world, that the quantum actors seemingly reject while developing many relations to its existing components.

Chapter 4 considers the most interior aspect of quantum computers, with what appears to be clearly a hardware issue: the development and definition of a qubit. This focuses on the central nonhuman actors in the quantum computing system. The chapter considers the agency of qubits, and the ways they are constructed and represented through a series of distinct accounts and actions that enable them to compute and connect with a variety of non-quantum actors. The chapter covers the many ways in which hardware is used to contrast the quantum and digital, and does suggest there is a significant novelty created by quantum nonhumans in computation. At the same time, the identity of these nonhumans and their relationship to the digital is multiple and redefined by non- 
hardware actors. The qubits ultimately emerge out of the cumulative interaction of all these efforts to distinguish digital and quantum.

In Chapter 5, I detail how software programming in quantum computing is developing by interacting with the commercial and social conventions of institutions, as well as the traits of the structure of hardware resources. The chapter follows the interaction of a team of researchers attempting to figure out how to program, and to be programmers for, a quantum computer. I argue here that software development becomes a negotiation point at which a problematic is formulated that then acts to shape and grow the network of quantum computing. The chapter follows the development of a problem and shows how it comes into existence and creates the relationships that will later permit programming. The domain of software is in this case indeterminate, coming into being through the agencies of distinctly non-software actors before familiar programming conventions are established. The chapter decentres many of the central topics typically focused on in software studies, such as the role of specific languages or functions, and proposes a view of programming based around a mix of social and technical problem-solving. The desire to maintain a clear distinction between quantum and digital programming plays an important barrier in this development. As the researchers view the problem with a more flexible approach to the problem-formulation process, it permits the viability of a hybrid computational model to emerge - a model that doesn't simply 'solve' problems, but which permits a new understanding of the problem-formulation processes to reshape 
how software application design is understood.

Chapter 6 takes a look at how the institutions involved in quantum computing have worked to grow the actor-network in particular directions, and how this has been accomplished by developing and spreading a shared mindset. The chapter shows how this mindset is in part anticipatory, helping bridge quantum computing into new areas even before it is able act in them. It is the anticipation as much as the actual information processing of quantum computing that permits the technology to spread as a mindset. This mindset attempts to stabilize the translations described in the previous chapters, but is tied to an uncertain future development process around those translations. This uncertainty in turn needs to be incorporated into the mindset of the corporate actors propagating it, for the actor-network to grow and continue to hybridize with the existing computational and corporate landscape. Its goal is not only to make the machine work in new ways, but to make new actors entangle themselves with the machine and its accompanying vision of computation.

In Chapter 7, I conclude by discussing my findings in the context of the patterns of rapid technological innovation established by digital computers, and reflect on the process of describing computation with a socio-technical framework. 


\section{Chapter: Socio-technical Approaches to Technology and Computation}

Socio-technical approaches to studying technology emerged as a coherent research agenda in sociology with the publication of Biker, Pinch, and Hughes' edited collection, The Social Construction of Technological Systems (1987). The essays it contains reflect an extension of the Strong Programme's effort to study how meaning is created in science. The Strong Programme applies a consistent social constructivist analysis to scientific activity and content (Berger and Luckmann 1966, Bloor 1976). This aims to challenge the separation of idealized knowledge production from social/professional activity found in sociological accounts of science (Popper 1963, Merton 1973). In response, the Strong Programme centers around four principles proposed by Bloor (1976): seeking the causes of scientific belief or knowledge, explaining scientific activity regardless of the success or failure of its results, considering these success or failures in the same way, and offering explanations that could be equally applied to the practice of sociology. This aims to understand science as a social activity, removing it from an isolated, idealized, naturalized, fatalistic, or otherwise uncriticizable realm of activity. Socio-technical work extends the Strong Programme's assertion that there are not simple, reliable, or merely logical paths from a positivist nature to scientific facts. It goes on to similarly call into question the path from science to technological constructions. Instead, creating technology is seen as an active social process that can be conducted in many possible ways. Socio-technical 
studies argue it is not the case that social factors only come into play at the end or late stages of an engineering process, distinct from a period of technical innovation. Right from the start, "technical, scientific, social, economic, or political considerations" are bound up into an organic socio-technical whole (Callon 2012, 123). This rejects histories and theories of technology that lapse into reductionist stories of the individual inventor-genius. Similarly, the socio-technical pushes against the characterization of technology as overly determining relations in society, common in philosophical, economic, or sociological theory (e.g. Marx and Ellul). This technological determinism had been contested or complicated in other traditions (Mumford 1934, Innis 1950), but remained a popular mode of critique at the time (see: Winner 1980).

The Bijker Pinch, and Hughes (1987) collection congeals an alternative approach in sociology that has influenced many other disciplines. Their book includes early versions of several varieties of socio-technical approaches: SCOT (Social Construction of Technology), ${ }^{4}$ LTS (Large Technical Systems), and ANT (ActorNetwork Theory). SCOT focuses on the variety of meanings that people hold about a technology and the way these meanings compete to shape technology. This tends to highlight the criteria being generated around a technology and the construction of a context which determines its apparent success or failure. LTS emerges from a more history-based examination of technology, and as the name

\footnotetext{
${ }^{4}$ Somewhat confusingly, SCOT is used to refer to the 1987 collection, its own group of theory/theorists, and in some cases the larger umbrella of socio-technical research that also encompasses LTS and ANT.
} 
implies, a concern with large systems or infrastructures. LTS identifies patterns of innovation, development, and system growth, attempting to place them in broader political and economic contexts (Hughes 1983, Summerton 1994, Kim 2005). As discussed below, ANT takes the most radical break from sociology, infusing it with concepts derived from semiotics and by proposing nonhuman agency. ANT describes how nonhumans act, resist, and negotiate with their more obviously social human counterparts.

All of these socio-technical schools of thought focus on materials and technology and their close entanglement with people. Their shared driving principle is that "society and technology are entangled together", and need to be studied and understood together (Bijker Pinch and Hughes 2011, xxiii). This rejects explanations based on the abstract or completely humanized social forces common to sociology. The socio-technical approach produces detailed examinations of these entanglements, and argues that without them an analysis will miss how technology and materiality mediate relationships between people, or between people and technology (Bijker, Pinch and Hughes 2011, xx). A similar outlook has been embraced in engineering, particularly software engineering, and described as "sociotechnical systems". This encourages the evaluation and design of technical systems by considering the broader context of actions taken by the people and organizations that use these systems (Sommerville 2011). While it also stresses a social-technical entanglement, it preserves distinct 
domains of social and technical objects, and tends to limit the time and extent of interactions between them to moments that can be optimized.

Of the SCOT-related approaches, ANT literature has developed an understanding of networks that most strongly seeks to attune socio-technical research to the social contributions of technical/material components. In particular, this understanding of how networks are built and grown focuses on the negotiations that humans must conduct with these components in order to develop new technologies, patterns of work, and stable social relationships. Below I examine this conception of network before considering how ANT has lacked a substantial attention to information processing. I then consider how this rich understanding of networks aligns with work done on the sociology of information infrastructure. Socio-technical principles have filtered into research in communications and media studies and the history of computation, and these studies have used this influence to develop the literature on computers and computation. I finish the chapter by examining how these have handled subjects that ANT has avoided. I explore how they can help fill the gap in ANT literature, as well as benefit from research on quantum computation.

\subsection{Networks and the Nonhuman}

ANT is a collection of analytical concepts initially developed by Bruno Latour, Michael Callon, and John Law, while they worked together at the École des Mines in Paris, during the 1980s. They developed these concepts in order to describe and circumvent their dissatisfaction with several prevailing tendencies in 
the social sciences, humanities, and technology studies. They felt that these areas of academia assumed too many unnecessary conceptual dualities, such as society/nature, human/nonhuman, and structure/agency. These categorizations had developed out of disciplinary traditions, and had a tendency to overemphasize human influence as the only factor in systems or relationships that included humans at all (Law and Hassard 1999, Latour 2007). Latour, Callon, and Law did not adopt the acronym of ANT to describe their work until a decade later, due to some hesitancy toward formalizing their methods (Law and Hassard 1999, Law 2003). Hesitancies aside, they instigated extensive debate about how to describe socio-technical networks. Here I examine how ideas of the nonhuman, negotiation, and of a 'messy' configuration shape their descriptions of networks.

\subsubsection{Nonhuman}

Early ANT studies describe how scientists perform science in contemporary and historical contexts, and argue for the agency of the "nonhuman" components that make this work possible (Latour 1988, Sismondo 2010). This claim, that the nonhuman can have agency, has remained one of the most critiqued and defended presumptions of ANT (Berker 2006, Sayes 2013). The relatively recent development of intelligent objects may make this seem to be a claim about hightechnology relevant to computer networks; however, the ANT notion of agency has little to do with intelligence or the essence of any particular object. The use of material objects, particularly technological artifacts, to preserve and shape social roles is a founding principle of all social activity. Latour points to common 
archaeological evidence that reveals the use of refined tools and other crafted objects predating the evolutionary distinction of homo sapiens from their biological predecessors. He suggests that this indicates the founding importance of technical artifacts in producing even the most basic of lasting social relations, and indicates further that the social is not a strictly human domain (1996b, 2012).

The human sense of 'being social' evolved in tandem with material culture, not independent from it, as objects have always co-produced the social. Technology is not a product of human social relationships, but rather a required contributor to them. Latour argues that simple artifacts act as a durable foundation for lasting social behaviours to develop, and more complex technologies similarly take part in forming new social relations as well as being formed by them (Latour 2012). This is reflected in perspectives advanced in evolutionary biology that consider the technical developments of humans as part of the natural selection process, not as an escape from natural processes or as a separate sphere of development (Latour 1996b, 2012, Dennet 1995). What we usually think of as social orders are instead always socio-technical orders, even when they are not obviously focused on a particular piece of new technology. Since Latour describes technology as the social made durable, then a socio-technical order could be understood as an order of less durable and more durable elements, or an order of varying social durability. 


\subsubsection{Negotiation}

ANT agreed with the main assumptions of social constructivism, which proposed that socio-technical systems arise through negotiations among people, institutions, and organizations - but ANT also added material artifacts as central figures in these negotiations. This addition began a decentring of the human subject as the sole topic of ANT's version of sociology. ANT claims that society or social relations need to be studied in a symmetrical way. Humans are clearly part of social activity, but equal attention should be paid to how nonhumans take part in that activity. The construction of either facts or technical artifacts, requires the activity of many nonhumans, and they must in some way be accommodated, or made to act productively, through a negotiation. ANT's use of negotiation departs from technological determinism, suggesting there are many possible ways to work with nonhumans, but also differs from social constructivism, by giving significant weight to the sway of materials and objects. This sense of negotiation was described in early ANT work in aggressive and militaristic metaphors of "allies and opponents, strategic negotiations, and tactical manoeuvres" (Keulartz et al. 2004, 14). However, negotiation does not require a tone of struggle and implied defeat for one side. It can just as easily be a process of finding ways to cooperate and get work done, rather than of competition and dominance. Negotiation can be boring and routine, closer to a quarterly employee review than a military triumph, particularly in a long-lasting network. However, it is not surprising that ANT put the negotiations of a network, particularly the difficulty of working with nonhumans, in these negative terms. 
Many key early studies centered on negotiations that failed, often with at least some fingers pointed at the inability to bring nonhumans under consistent control. A classic example is the role of batteries in the failure of the electrical car network that Callon describes in the SCOT collection (1987) (see also: Callon 1986, Latour 1993, Farias and Bender 2009).

Creating a nonhuman/human categorization that determines which participants in society count, and how they should be counted by researchers across all contexts, is seen in ANT literature as an unproductive discrimination that tells little about how social relations are performed. Latour, Callon, and Law argue that the human-centric approach to sociology creates a gap between social theory and observation. They claim that careful observation of social activities in practice does not reflect the human-centric theories of social behaviour. Focusing only on humans as the exclusive actors in society does not seem to account for the cumulative social result, and neglected the many relationships between people and objects that constitute a great deal of modern social life (Latour 1985, Law 1992, Law and Hassard 1999).

Instead of referring to humans and nonhumans as such, ANT substitutes its own terminology to encourage a symmetrical view. An "actor" may be a person, an organization, an animal, a chemical, a technological artifact, or any other entity that other actors associate with, relate to, or are influenced by. Though it may seem tautological, what constitutes any one actor in practice is decided by other 
actors through their interaction and a process of "translation" (see Chapter 3 for an extended discussion of translation). Actors are simply anyone or anything that act upon others. Latour seems comfortable in moving the "A" in ANT between the terms actor, agent, or actant rather freely $(1987,2004,2010)$. Intentionally vague distinctions of these variations were introduced in order to emphasize that researchers should assume as little as possible about any essentialist characteristics belonging to the entities they are examining (Hache and Latour 2010).

In some circumstances, however, actant has been used to retain a difference between the actors that can speak for others and actants which act without the ability to speak or broadly campaign for their own vision of how the network they are part of should operate. Usually, this means that actants are typically nonhuman, though this is not consistent. The term actant is borrowed from Greimas, who uses it to describe the basic unit of structural-semantic analysis for understanding narrative and myth. Actants in narratives provoke action, but are not required to be a personified character. Instead, they are defined by their actions and contributions to a story (Greimas 1970). No actant always has the same predictable effect. Its arrangement relative to other actants can produce different narrative outcomes. Actants and actors are likewise defined more by their functionality and relationships than by common essences with other entities of a similar categorical type. Actors can be individual, collective, figurative, or nonfigurative (Latour 1988, 252). 


\subsubsection{Messy Networks}

In the workshops that eventually became the SCOT (1987) collection, Callon coined the term "heterogeneous associations", which has been a popular phrase for emphasizing the plurality of types of actors in a network, and the many ways in which they can connect and influence one another (Callon 1986, Latour 2005, Dolwick 2008, Cressman 2009). The difficulty in trying to trace, map, or explain a network with a near-infinite amount of possible subjects and processes, has been a point of repeated reflection by ANT's founders (Law and Hassard 1999, Latour 1999, Law 2004, Latour 2005). Socio-technical networks may create a sense of order in society or allow an operation to occur, but themselves may not be governed by definite, stable, and repeatable processes (Law 2004). Making it worse, the negotiations that establish all these associations never quite close. Networks are messy, and not just the ones that break. As an increasingly heterogeneous group of actors are distributed agency in a network, the more aberrations or transformations in the network's operations can occur without actually collapsing the network. Networks are messy because their default is a constant state of flux. A mess is not necessarily random, but it is hard to describe, predict, and envision for those who are in its midst. ANT tries to highlight the messy nature of networks, as it is often erased by other accounts, especially those of working systems (Law 1993, Law 2007). Law in particular argues that ANT's contribution is "a sensibility to the messy practices of relationality and materiality of the world" and the wariness of large-scale claims common in social theory $(2004,2007)$. Messy networks can be read or organized 
by a researcher in multiple ways, "realities, messy or otherwise, are enacted into being" (Law \& Singleton 2005, 334). ANT's acknowledgement of research objects as messy, breaks apart any notion of their singular existence, creating a challenge to study objects as existing in multiple worlds (Law and Urry 2004, Gavan 2007, Kien 2016).

ANT is not the first theory to notice that material culture plays a role in social activity and communication. However, when they insist "almost all of our interactions with other people are mediated through objects" (Law 1992, 381-2), it goes a step further than other sociological and media theory in arguing that the human/nonhuman is a barrier which must be constantly ignored (Couldry 2004, Dolwick 2008, Sismondo 2010, Tatnall 2010). The most popular concept to come out of ANT is that of the actor-network, with it being deployed by a wide range of fields outside sociology (Turner 2005, Tilson and Lyytinen 2005, Shiga 2006, Pots 2008, Rumming 2009, O'Connell, Ciccotosto, and De Lange 2014). This broad application reflects many authors seeking ways to analyze interactions between people and objects that consider them as equal factors in the creation and functioning of social processes. Actor-network is presented as a hyphenated term to emphasize the uncertainty around the "origin of action" being described (Latour 2012). An actor-network account tries to find out how a coherent whole is produced by ongoing and fluid relations between actors. In this way, the actornetwork has similar attributes as that of an assemblage (Deleuze and Guttari 1980, DeLanda 2006), a comparison Latour has embraced $(1999,2005)$, due to its connotations of transformation, relational identity, and construction. Actors 
interact to make up a network, but an actor is also "one output of the relationship in a network" (Cordella and Shakh 2003, 4). Connecting the actor-to-network implies a flow of actions about which we should not assume a clear preexisting relationship. The actor-network represents a continuum where networks are made up of actors, and actors are made of networks (Callon 1987, Latour 2012).

ANT's conceptualization of networks contradicts the dominant understanding of the term in many fields. In particular, ANT was developing at the same time as popular information and communication technologies (ICTs) were being transformed by digitization. The changes to ICTs drew interest to the characteristics of various technical networks and their effects on globalization. Castells provides a minimal but influential definition of a network as a series of nodes connected by links, "where a node is the point at which a curve intersects itself" (Stadler 2007, 169). This abstract definition is broad, making the notion of networks flexible. Evoking similarities to ANT, Castells suggests that characteristics of specific networks are determined by their empirical material qualities (Castells 1996). However, Castells' research is specific to the impact of the information technology revolution and its structuring influence on social organization. Latour describes Castells' use of the term as a merger of technical networks with the sociology of organization's differentiation between organization, markets, and states. In this combination, "network becomes a privileged mode of organization thanks to the very extension of information technology" $(2005,129)$. 
The common understanding of networks, including television, electrical, sewer, or social media, are not required to be the object of study in ANT. Rather, a network is a conceptual tool for basic issues of social theory, epistemology, and philosophy (Murdoch 1997). A network in ANT has also been described as a "worknet" to draw attention to the labour being performed or the mediation taking place to create and maintain actors (Latour 2005, 132). Actors are not nodes along pathways of networks, but rather the effect of work that holds together to construct an identity. The relationship of ANT to other network theory has been confused because of these differing uses of network. Early ANT studies also involved technical networks as actors, further confusing the issue (Latour 2005, 128-132). However, ANT's concept of the network provides a useful way to reexamine technical networks, like those that Castells describes, and view them as active processes of social work and relationship building between actors that are both on and off the technical schematics of passive nets and nodes. Throughout the following chapters I frequently use the term network in place of actor-network. Whereas Latour and ANT literature worry that on its own network is too connotated to ICTs, and implies a flat communication process in which there is no transformation or ongoing construction (Latour 1999, 2005, 2012), I find network less troublesome as a term, and read it as able to include the flexible and transformation-based processes described in Chapter 3's examination of translation. 


\subsection{ANT and Critical Computation}

Nonhuman technological actors have been the main focus for much of ANT because they have been traditionally overlooked in sociological theory. ANT argues that a variety of actors labeled as nonhumans are the "missing masses" that make society function; treating nonhumans "symmetrically" as social actors is supposed to cover the human-centric blindspots that hide how the social is made possible (Bijker and Law 1992, 225-229). This allows us to trace the "mechanics of power" native to each network (Law 1992). This section explores ANT's lack of attention to computation, and considers its use of matters of concern in this context.

ANT's perspective treats its objects of study as effects that are created by the network of relations in which they are located. Studies with this approach explore and describe these networks, and are often especially concerned with how changes in relations between actors reshape or destroy entire socio-technical systems. Its concepts are well suited to examining the consequence of connecting new technical and social actors together (Latour 2004), a feature which would seem to make it fitting for the growth of computational networks/strategies which have come to interact and shape virtually every social action.

Yet, there has been little attention from ANT studies to the actor-networks of computation. Instead, computers and computer networks have been focused on as methods that enable the distribution of work/agency across diverse and 
distant actors (Kelly and Maddelana 2015). Collectives of humans and computer technology are occasionally studied by applying actor-networks, such as in the context of video games and virtual environments (Cyper and Richardson 2006). These point to the ways in which computers help construct hybrid agents that are "human and non human, social and material, corporeal and technical", focusing on the power of the digital computer to facilitate new kinds of hybrid actors and spaces. Tatnall's (2010) excellent edited collection brings together many insightful applications of ANT with high-tech subjects. These studies look at how digital tools are used, and often fail to work with other human and nonhuman actors as expected. Such studies expand research on ICTs, though tend to blackbox or avoid discussing the development of computation or the innerworkings of the digital actants they describe. For example, Sekgweleo and lyamu's chapter on the distribution of computer software in a South African telecommunications company, suggests how different understandings of the problems to be solved in an organization become based on overly social or overly technical models of their operations. They show how this disrupts the everyday deployment and implementation of software. Such studies are valuable, and intentionally flip the classical ANT symmetry tactic, by aiming to insert humans into accounts of networks that have been overly technologized. However, they tell us little about software or computation as an actor-network itself.

Few have taken notice of Latour's (2010) minor suggestion that computer programming operates analogously to the functions of an actor-network, or 
sought to widen that remark to the more heterogenous computational interactions beyond software, to the full breadth of actors generating and shaping systems of computation. Latour (2004) also invokes Turing and the creation of the computer in the last paragraphs of his well known critique-manifesto, "Why Has Critique Run out of Steam?:"

Turing too cannot avoid mentioning God's creative power when talking of this most mastered machine, the computer that he has invented. That's precisely his point. The computer is in for many surprises; you get out of it much more than you put into it. In the most dramatic way, Turing's paper demonstrates, once again, that all objects are born things, all matters of fact require, in order to exist, a bewildering variety of matters of concern. (247)

What is "put into", what you "get out", and the matters of concern at play in the computer he leaves to the reader to assume. This reader would like to know what they are! Viewing the computer as a matter of concern implies examining their many participants, historical contexts, and how computation becomes engaged with its own inherent contradictions and controversies (Latour 1999, 2004, Riple, Thun, and Velikov 2009). Latour sees the computer and digitization "not so much an innovation as the achievement of a three centuries' old dream" to make all phenomena measurable and manipulatable $(2008,47)$. Many traditions, people, and technologies are 'gathered' to make computers able to represent and manipulate the world, by developing and mechanically embodying problems they 
can then process. Latour's matters of concern direct us to see how the many Heideggerian things required for an object, such as a quantum computer, are brought together. This would emphasize the desire that creates the gathering process more than the resulting structure. This means understanding the worldview of problems a computer enables. Developing concepts that can help to interpret and model computation as an actor-network in this way, has been little explored.

An interesting exception is Pavlovic and Meadows' Actor-Network Procedures (2011). This article proposes the actor-network as a way to describe "the coalitions that are formed between humans and devices in order to enable secure computation and communication" (2). This attempts to bring human actors and non-mechanical processes into the framework of computation, and make mathematically precise descriptions of their commingling. Their account is also a model for computing the equal actions of humans and nonhumans in the context of operating security protocols over computer networks using multiple channels. This is motivated by a perceived need to replace a more limited computing framework for such protocols. They specifically deal with two-factor identification. Their definition of an actor-network is unique in the literature:

An actor-network consists of the following sets: (1) identities, or principals $\mathrm{J}=\{\mathrm{A}, \mathrm{B}, \ldots\},(2)$ nodes $\mathrm{N}=\{\mathrm{M}, \mathrm{N}, \ldots\}$, (3) configurations $\mathrm{P}=\{\mathrm{P}, \mathrm{Q}, \ldots\}$, where a configuration can be a finite set of nodes, or a finite set of configurations; (4) channels $C=\{f, g, \ldots\}$, and (5) 
channel types $\Theta=\{\mathrm{T}, \zeta, \ldots\}$ given with the structure $\Theta \vartheta \leftarrow C \delta \Rightarrow P$ $\rightarrow \mathrm{J}$ where the partial map @: $\mathrm{P} \rightarrow \mathrm{J}$ tells which principals control which configurations, the pair of maps $\delta, \rho: C \rightarrow P$ assign to each channel $\mathrm{f}$ an entry $\delta \mathrm{f}$ and an exit $\rho \mathrm{f}$, and the map

$\vartheta: \mathrm{C} \rightarrow \Theta$ assigns to each channel a type.

An actor is an element of a configuration.

While the symmetrical human/nonhuman thinking behind this proposal does align with some ANT concepts, and provides a unique translation of ANT, it greatly departs from the perspective and intention of ANT literature. The paper's outline of communication, for example, removes the transformative processes at play in an actor-network and reads closer to Shannon's (1948) transmission model in its definitions: "Communication in a network consists of information flows along the channels. Each flow corresponds to a pair of events: (1) a write event at the entry of the channel, and (2) a read event at the exit of the channel" $(7,2011)$. The rigid formalization of the paths and actions actors can take by the mathematical model severely limits its compatibility with the actor-network approach. However, the authors' motivation to look to ANT and consider its foundational assumptions is compelling: "we contend that computation and society have pervaded each other to the point where computer science and social sciences already share their subject" $(2,2011)$. 


\subsection{Critiquing Actor-Networks}

Some of the most thorough, or at least philosophical, critiques of ANT come from Latour (1999, 2005, 2010) and Law (Law 1999, Law and Hassard 1999, Law 2004) themselves. They have tried to offer several correctives to ANT as a theory and method as its popularity increased, and in response to several waves of criticism. Latour has distanced himself from ANT (1999) only to later embrace it again (2005). Putting aside their own unease with the codification of the principles and concepts they have described, several issues have led to persistent criticism.

The implications of describing nonhumans as having agency, and whether agency implies intentionality or self-awareness, was an early flashpoint of academic scrutiny around ANTs ontology. Gad and Jensen (2010) point out the fundamentally different assumption about the nature of agency that underlies this critique: "The ANT concept of actor does not match the sociological concept of agency because it does not refer to actors with predefined characteristics but rather underscores the many ways in which actors bestow agency on one another" (Gad and Jensen 2010, 61). This differs from SCOT, where technological artifacts are assessed by looking at human statements, rather than as actors with their own existences and role in constructing the identity of other actors (Law 2003, Bijker 2010).

Assessing agency in ANT becomes a process of describing distributions of abilities across as many media as are required to make a network function. 
Describing such distributions raises two key problems. First, there can be an illusion of symmetry, as nonhumans still must be accessed through those who can speak for them. Early in ANT's development, Colins and Yearly (1992) pointed out it was not possible to get an account of a controversy or state of a network directly from any nonhuman actor. Instead, since human spokespersons were always mediating to some degree, the topic was ultimately a matter of social construction, which couldn't be escaped by appeals to symmetry.

Schulz-Schaeffer suggests an erroneous claim of symmetry can be compounded by a lack of technical expertise, which becomes a handicap for many individuals trying to employ ANT when studying objects with which they only have a superficial technical knowledge (Passoth and Rowland 2008, 835). This may be true in many circumstances. As can be seen in cornerstone ANT studies, a great deal of technical knowledge is required to pursue the "material ethnography" ANT prescribes (Callon 1986b, Latour 1988, Dolwick 2008). This can also lead to situations in which the generalizing language of ANT (e.g. actor, network, agent, artifact) is used to obscure either a lack of deep technical knowledge or can interfere with the actors providing their own terminology and descriptions to the researcher (Chateauraynaud 1991, Lee and Brown 1994, Latour 1999).

Second, ANT has been criticized for producing its "thick descriptions" while ignoring the political aspects of their contexts (Sturman 2006). Benson (2014) labels this the "new descriptivism", highlighting a concern that ANT descriptions lack the ability to provide explanation and prediction. Benson and others 
acknowledge that prioritizing description over explanation is an intended feature, rather than a bug, of ANT (Law 1992, Law and Hassard, 1999, Bijker 2010, Benson 2014). The concern with context and explanation is, however, increased by the accompanying impression that as ANT tends to focus on how actors make work happen, it can appear to be privileging a managerial point of view (Dolwick 2008, Couldry 2008). This is particularly relevant when dealing with the worldview of high-tech capitalism, whose power structures and ideologies centred on rapid-change and innovation, may be masked by a flat ontology focused on project-based localized actor-networks (Benson 2014). Addressing this criticism requires not depicting networks as in an unchallengeable form. Thick descriptions can also undermine managerial narratives of control and planning, highlighting the agency of actors not always credited with power and influence.

A similar concern has been raised about the lack of attention to the role of gender in the technical and scientific spaces so often described by ANT (Sturman 2006). For Sturman, the neutral treatment of gender, and its potential erasure through ANT terminology, poses a significant problem to ANT and Latour's approach in particular. The lack of attention to the construction and agency of gender in these spaces is similarly accompanied by a lack of attention to race. Attention to such factors and their construction could certainly enrich ANT case studies. Interestingly, the way out of this descriptive dilemma for Benson, is the same proposed by Latour and Law: by using ANT as a way to open up new objects of study, and then hybridizing it with other perspectives. 


\subsection{Socio-technical Infrastructure}

ANT acknowledges the disagreement and breadth of possibility open to a technology at its early stages, and suggests the need to trace how controversial technologies change and grow - addressing, shifting, or ignoring expected goalposts along the way. The decision-making, failures, and discoveries that shape this growth need to be uncovered in the laboratory, business, and many hybrid spaces in which they occur. These are spaces rarely examined in detail or given priority by the accounts of computer development explored later in this chapter. This growth is not an easy thing for a researcher to observe, and certain kinds of moments and narratives tend to emerge. This has led to a particular focus on moments of failure and controversy.

The socio-technical view of ANT and other SCOT-like approaches frequently stress the non-deterministic state of any technology. Objects, institutions, or societies could always be configured differently. The failure of a network reveals this nicely, and implies a sort of respect, or perhaps bewilderment, at how other networks have become so durable given the tentative state of any socio-technical relations (Callon 1986, 1987, Law 1992, Law 2004, Latour 2005, 2010). Failures and controversies also tend to be sudden events that occur over a limited period of time, making them more practical to study and describe. Chapter 3 describes this directive of ANT to use failures and controversies as entry points in describing socio-technical networks, which has been a frequent tactic to open up networks to analysis since Callon's famous study of scallops in St. Brieuc Bay 
(1986). However, the ANT literature that recommends this has little to say about the limitations it may incur (Law and Hassard 1999, Venturini 2010).

This approach of focusing on moments of failure or controversy has also been taken up by literature on sprawling or well established technical networks and infrastructures, which results in an emphasis on the visibility/invisibility of infrastructure. These studies privilege disruption, failure, and breakdown as conditions that disclose our dependency on its operations, revealing the naturalization, ubiquity and convergences of technologies that run in the background (Urry 2004, Sheller and Urry 2006, Edwards 2010, Pinch 2010, Parks and Starosielski 2015, Starosielski 2015, Peters 2015).

However, literature on large technical systems (LTS), technical networks, and the sociology of information infrastructure has tended to pay more attention to early and expansive moments in technical development. Hughes' (1986) well known description of large infrastructures developed a view of technology focused on entrepreneurs and engineers. This approach stressed the steps required for spreading a technical network and the problem solving required to do so. This echoes earlier considerations of infrastructural development around the recognition and solution of problems, and how the problem-solving process created new organizational structures and professional classes (Chandler 1977, Beniger 1989). 
Hughes elaborated an account of technology as composed of "problem-solving systems" $(1986,47)$, which permits a more critical assessment of the way technological systems implicate people in the ceaseless activity of problem solving that allows the surrounding environment to be exploited for potential solutions. This attention to the definition, construction, and transformation practices around what is understood as "the problem" is highly relevant to quantum computers (see Chapter 5). As non-universal architectures pioneer the paradigm and engineering practices of quantum computation, the problematic becomes a central actor in this new quantum terrain alongside human and other material nonhuman agents.

Hughes' (1986) description of technology as a "seamless web" or network, challenged the reality of theoretical divides in practice; "technology and science, pure and applied, internal and external, and technical and social, are some of the dichotomies foreign to the integrating inventors, engineers and managers of the system- and network-building era" (Hughes 1986, 287). Hughes argues these dichotomies linked unlike actors, including physical artifacts, firms, utility companies, academic research, laboratories, and banks, into diverse webs to meet the managers' own goals. He notes how instigators of these webs aligned the interests of each participant into a single system $(1986,287)$. This alignment is similar to how ANT would describe actors' interests as having been translated into a "program of action" (Callon 1986a). Hughes notes that "environment becomes organism" and "background merges with foreground," meaning that in the creation of technological artifacts, the social, natural, and political contexts 
they interact with are embodied in the technology, or at least in successful technologies (Hughes 1986, 290-1).

In the 1990s, LTS shifted to de-emphasize people to focus on infrastructural relations (Star and Rhueder 1996), and the sociology of information infrastructure developed around the work of Susan Leigh Star (1995, 1997), Geoffrey Bowker (1994), and Paul Edwards $(1988,2010,2013)$ as a phenomenological or materialist turn away from the Hughes' LTS approach. In their collection on media infrastructure, for instance, Parks and Starosielski (2015) distinguish their materialist approach from Hughes' work in these terms, and John Peters' new book (2015) offers a similar justification for situating infrastructural scholarship with respect to the legacy of phenomenology.

Edwards (2010) provides a thorough study of information processing infrastructure in the context of climate science. He describes a process of "infrastructural inversion" in terms of breaches, violations, breakdowns, accidents, or glitches that disclose the normal operations of infrastructure. Like early ANT work, this traces the relationship between the work of scientists and their models, data, and environment. Edwards' account aligns with Bowker's (1994) initial use of "infrastructural inversion" in Science on the Run, and this encourages a different vision of information infrastructure: as a site of ceaseless inversion through problem solving that is conducted to gain knowledge of perpetually uncertain circumstances. Edwards (2013) similarly addresses changing knowledge infrastructures. Here he traces how information processing 
and distribution are transformed not only by new technology, but by the development of institutions that set routines, establishing information sharing traditions, and possible directions for a network to grow.

Bowker's work repeatedly turns to the role of the environment and the impact/manipulation of material conditions on the formation of technical networks (Bowker 1994, Star and Bowker 1995, Bowker and Star 2000, Kaghan and Bowker 2001, Baker, Bowker and Karasti 2002). Like Edwards (2010) this traces the interchange between the materials, the production of data, and the act of theorization, all of which are necessary to form and operate technical networks. This approach to studying technical, often large, networks has been followed by work such as Starosielski (2015), which takes the materialist turn, but incorporates it with broader environmental descriptions as well as a range of other human and nonhuman actors. Peters (2015) broadens this infrastructural account even further, with an emphasis on the technologization of the environment, and the ontological properties of computational media.

\subsection{The Computer}

Media and communication studies offer a number of useful resources for thinking about computers and computation, in terms of their mythologies, history, and theory. Taken together, these offer examples of the heterogeneity of associations at play in computation, and the breadth of it as a matter of concern. Given the lack of ANT writing on these kinds of actors, it provides an idea of the relations, 
and their negotiations, that would need to be addressed by a socio-technical account of a computational network.

Carey described the adoption of digital computers as an extension of patterns seen with the introduction of other new communication technologies (i.e. the telegraph): growing government and military resources, opening more time to commerce, controlling activity in space, and generally coordinating life in the post-industrial society $(1989$ 12, 20, 21). For Carey, computers are an example of the deep relationship between technology and ideology (21), as they are accompanied by powerful myths about their social impacts, such as expanding democracy, efficiency, or harmony. Mosco further describes this process of mythmaking with technological innovation, noting the "historical amnesia" (2004, 8) shown by technology prophets who proclaim great social changes will be tied to the spread of new technological devices. These changes have progressive techno-utopian overtones, which hide the unequal power relations that they reinforce. Computers in this perspective are ways to extend and intensify existing logics of capital and governance by embedding them in a rhetoric of personal or societal liberation typified by thinkers like Kurzweil (1990). Carey sums this dynamic in his allegory of the "microcomputer", now called a PC, becoming the emblem that replaced the watch as a workplace retirement gift symbolizing social contribution and opportunity (21). 


\subsubsection{History}

Hardware dominated early histories of digital computing, and tended to focus on particular devices, classes of computer, and their inventors. This has transitioned to increasingly focus on software and institutions, and incorporating a sociotechnical approach that considers a range of relationships as the subject of computational history. Traditional computer histories depict a lineage from brief accounts of primitive calculating devices to detailed descriptions of electronic digital computers, a trend that has continued in contemporary descriptions (Shurkin 1996, Ifrah et al. 2000). This trend prioritizes a history of innovation and of the technological perfection of electronic computing. Famous examples of individual machines and their inventors, such as the ENIAC, have dominated many histories (Redmond and Smith 1980, Metropolis, N., Howlett and GianCarlo Rota 1980, Williams 1997, McCartney 1999). Similarly, the spread and traits of the personal computer tends to be heavily emphasized (Friberger and Swaine 1999), likely due to its familiarity.

However, by the 1970s "software had become the computer" and was the subject of its own histories (Ensmenger 2010). Campbell-Kelly (2007) describes how histories of software began as descriptions of technical systems. From the 1950-1970s, these focused nearly exclusively on coding and software engineering practices. He suggests this focus has remained with many newer critical histories, as it lends itself to familiar modes of historical textual analysis and literary criticism. In the 1980s, software histories expanded to include 
economic narratives and business histories (Modis and Debecker 1988, Chandler 2001, Campbell-Kelly 2007, Malerba, Nelson, Orsenigo and Winter 2008). This has also frequently adopted a narrative of pioneering figures, overly highlighting the accomplishments of a handful of already well recognized successful individuals and companies (Ensmenger 2010, 3).

Yates (2005) expanded these accounts of the software industry to consider how corporate users have shaped computer development. Her research highlights the relationship of IBM to large insurance providers, showing how particular early corporate users with massive amounts of data at their disposal adopted and shaped information processing technology through most of the twentieth century. Yates' analysis is seen as popularizing a broader view of the social relations that shape computation (Campbell-Kelly 2007), and offers a timely nudge given the development of algorithmic structures, particular neural networks and machine learning, which rely on huge amounts of data to learn/train their performance. The impact of the uses/problems of large users with unique datasets and resources, has created histories of military (Edwards 1988), corporate (Yates 2005) and government-bureaucratic (Agar 2003) organizations and how they shape or even explain current systems of computation.

Ceruzzi $(2003,2012)$ provides an account of the development of digital computation that works to integrate hardware and software histories. This is a useful foundation for understanding computation technologies, though it does not 
address the paradigm shift presented by quantum computing and hybridquantum systems. He argues that across historical moments, computing manifests into four themes: the digital paradigm, convergence, solid state electronics, and the human interface. These are useful themes to consider in the emergence of quantum computers; however, the quantum example expands and complicates each of them. The assumed victory of the digital over the analog, common to such histories of computation (Ceruzzi 2012, xi), is now also thrown into question. Ceruzzi points out that the dominant histories of computing have tended to be organized chronologically into a few standard chapters: of IBM and its rivals; to the evolution of the internet and web; and most recently to a focus on spreading networks, smartphones, and mobility $(2012, x)$. Taken on their own, these histories can be somewhat misleading as they often ignore the continued presence of previous computing chapters.

In contrast to such accounts of unfolding hardware and software systems, Turner (2006) examines how the social politics, ideology, or limitations of the humans involved in technical networks shape their outcomes. His historical analysis of Stewart Brand and the Whole-Earth Network takes a closer look at the specific social visions that became attached to information technology, even as these visions don't necessarily reflect the daily reality of their use. These user-focused histories show the integration of computers as important tools and cultural objects for organizations outside of business and government, while also linking these countercultural organizations to their capital-counterparts. Such cultural- 
historical accounts provide a fuller picture of the influences at play in technical networks and are useful for balancing out those who dive deep into the technical architecture, shifting away from the form of the machine as a complete explanation for its implementations and associations.

As Edwards (1988) points out, developments in computation have tended to be historicized into either a history of ideas that follows a progression of information concepts, symbols, and logic, or a history of calculating machines, whose progress creates new social and economic impacts. Bennett (1988), for example, largely ignores machines to describe the development of computing as the principles of thermodynamics being translated into models of information processing. This tells a history of ideas of famous scientists like Maxwell, Szilard, and Brillouin. This viewpoint is also seen in histories of specific computational techniques, such as evolutionary computing, which are posed as largely independent of any machine (Fogel 1998). The alternative is seen in narratives of machines, and tends to stress how available hardware shaped or determined the norms of computing practice (Grier 1996).

Edwards (1988) suggests a third approach, which follows computer development as a series of technological choices, which are made at many interwoven layers of relationships between people, organizations, and institutions. This approach can be seen in Mahoney $(1988,204)$, who draws upon SCOT to decenter the machine in his accounts, while tracing how communities of practitioners inscribe 
their experience and models of the world into those machines. This views the history of computing as a series of decisions about what is computable. Mahoney $(2004,2005,2008)$ suggests that the role of the critical historian of computing is to not recount a stable hardware, software, or organizational object, but to "follow the efforts to achieve a consensus" (2004) around what counts as software engineering or other computational work.

Ende and Kemp (1999) also adopt a socio-technical view to look back at the choices behind the "regime shift" that took place to establish modern electronic computing. They examine how novel traits of new technology transformed the strategies, research agenda, user practices, and engineering expectations established by prior computing regimes. This approach critiques the glossed over expansion of computing in historical accounts, which often neglects how the digital computer diffused into specific domains. Ensmenger (2010) suggests such SCOT-inspired approaches help computer history address the neglected or marginalized actors in accounts of computation, noting that software histories increasingly embrace the exploration of "heterogeneous associations" (8). For example, he uses this approach to highlight the various maintenance workers that enable the information age. This brings to light actors outside of usual categories of depictions of prime movers, innovators, or power-centers, to highlight how computational networks are also produced by the invisible, inglorious, work that holds them together. 


\subsubsection{Media Theory}

Media theory also has a rich history of describing the processes of mediation and the recruiting of new modes and materials to process information. Though at times there is a tendency to formalism, this is also coupled with a strong rejection of technological determinism (Smith and Marx 1994). McLuhan (1964) had an expansive view of what he considered information technology. He discusses computers only briefly as examples, with a focus geared toward "electric" media rather than a clear conception of the digital or of computation. Still, his examples of computers offers several avenues to conceptualize them: as media of communication along with speech (53), as extensions of human identity similar to clothing (192), as translators of actions into data (63), and whose memory may contain/simulate the environment (71). He verges on the techno-utopian when considering the ability to "transfer consciousness" and the computer's promise of "universal understanding and unity" with instant information (154-155). What can be taken away from his collective observations is an early consideration of the role of computers as simulation devices able to model complex social dynamics, and the role of algorithmic modeling and programming as the medium's message (291). The computer as a physical device is only vaguely touched on, but the recognition of it as entailing a process of transforming and representing the world into manipulatable information is clear.

Kittler (1992) acknowledges a Turing Machine conception of computing, that can be implemented by any number of media, including humans without the use of machines. However, most of his focus is on programming languages, as they are 
found in digital personal computers. His analysis develops into a detailed consideration connecting textual languages and material components that acknowledges the importance of a medium's design and construction. His deconstruction of the computer down to its "switching components" is an explicit critique of the digital/binary nature of the machine, and its ability to process and simulate the continuous non-binary world (154). The form of the computer is seen to prescribe its applications and effects. This is for Kittler part of "the price we pay for structurally programmable machines" (155); however this negotiation is reopened in the case of quantum computers.

Hayles (2005) further considers the computer with a focus on the linguistic and textual, using those elements to build up the understanding of text as performative. Code is writing that acts on both machines and humans, linking them together into a regime of computation. Hayles considers how computers are able to represent the complexity of the world as an emergent property of the "labor of computation" (41), the difficulty of which is in aligning the various layers of code to be legible to one another. While she acknowledges the arbitrary split between hardware and software, her writing reflects a shift in the field to a preference for software and the familiar ground of language, though one that acknowledges the interplay of a text with its physical materiality $(2004,72)$.

The layering of programming in hardware and software that makes each level both seen and unseen, as described by Kittler, is considered in depth by Galloway (2012) in the process of the interface. Rather than a media object, 
Galloway makes the computer nearly synonymous with mediation. The computer is described as a practice rather than an object, "a process or active threshold between two states" (23). As interfaces, computers serve to distinguish one level, content, or material from another, leaving Galloway to consider the computer as ultimately an ethical device. It is a process of segregating realms, types of actors, into kinds. Galloway doesn't always go into great detail about what each of these layers or kinds looks like, though when he does it's clear he's often referring to layers of code in software.

Software coding has been an understandable focus for researchers working in fields that have long studied languages, information, and semiotics. Manovich (2008), citing Innis and McLuhan as inspiration, brings media studies and computer science together to appreciate the development of software as a newly mediating layer across contemporary cultural production. Manovich espouses a practitioner-theorist point of view that describes code disconnected from any particular hardware. He builds upon the understanding of computers as remediation machines and universal simulators to highlight the unique results of the multimedia they produce and embody. He narrows this description of computer development to their influence on the cultural industries and the history of such "cultural software" that produces media content and formats. Manovich's push for dedicated software studies has been followed, and accompanied, by several like-minded scholars with a deep working knowledge of how code works with digital machines. 
As Fuller's (2008) edited collection demonstrates, this current writing on computers tends to focus on particular software applications, often in the context of mobile devices, and presumes an ICT model of connections and transmissions around or through software. Building off of work by Kittler (1992) and Hayles (2005) to address the interior operations of computers in analysis, software studies produces work that deftly considers code as an infrastructural element of daily life and highlights neglected features in computation. Software studies also tended to focus on free software or well-established components, and has had little to say about the cutting edge of computing development in theory and industry. This focus on free software, company ethics, hacker-culture, and consumer/user activities is easier to find in academic accounts of the social role of coding, such as in Wark (2004) and Coleman (2013); however, it has narrow applicability to the current state of quantum computing as a remote field of knowledge or of hardware development. This approach begins to bridge the language-based analysis of software with an interest in its structures, such as functions, and especially the algorithm.

Algorithm can be an excessively broad term describing any "series of steps", with many potential applications (Gillespie 2016). Its discussion in communication and media studies has been less focused on mathematical examples or definitions, but rather on "algorithmic systems" that include people, companies, data and code (Seaver 2013). Algorithms are often viewed critically as an unwanted form of mediation of social activity, overly controlling social actions and negatively impacting individual agency (McGee 2011, Steiner 2013, Pasquale 2015). In 
reaction, others depict algorithms as creative sites of cultural production (Steiner 2012, Manke and Uprichard 2014). They have also been taken as an opportunity to examine the conceptual divides and connections between notions of technology and culture/society (Goffey 2008, Striphas 2014). Some scholars have also sought to develop means of evaluating the social harm/benefit of different algorithms (Gillespie 2014, Kitchin 2016), while many have looked to study the embodiment of algorithm in code (Ziewitz 2016). In many studies to date that focus on algorithms, they are depicted as foundational to computing and extremely powerful, but also as inexact actors often with unintended consequences in broad and messy technological networks (Goffey 2008). This is highly relevant to the development of quantum computing. Yet, it seems useful to distinguish between the performance and manipulation of algorithmic systems in established computational systems, which have guided the critical discussion of algorithms thus far, and the logic of assembly of such systems. The development of algorithms in quantum computing requires some fundamentally different assumptions than the digital systems typically examined, but also shares many of the same traits and concerns of scholars concerned with the social role of algorithms. While I do address the process of algorithmic development in quantum software, I distinguish it from the relations underlying/constructing quantum computation to avoid an overly general use of the term (see Chapter 5's discussion of problematics), that would conceivably encompass all stages and steps of computation (Barocas, Hood, and Ziewitz 2013). 
Computers have become commonplace if not ubiquitous in everyday life. Their constant presence and circulation has created a focus on what is done with them as established actors in the world. As quantum computing challenges the fundamental paradigms of computing to date, it invites a rethink of how we conceptualize computers' technological development before they enter broader social processes. Instead of a focus on their cultural representation, militaryindustrial origins, inherited media legacies, or audience interpretations and appropriations, my account aims to open to view the incubation period of technological development and change. This can help to understand how this new technology is being made to exist in the world.

\subsection{Literatures to Methods}

The closed world of quantum computation, with the unique intellectual and financial resources required to enter it, marks it off from most accounts of computing today. Quantum computers require a careful look at what is being brought into existence to make them function. This helps us better understand their implications for computing, and what their formulation can tell us about how to understand and study computation more broadly. Drawing upon examples from critical studies of computation, information processing infrastructure, and histories of computation can help inform an ANT study of quantum computing. These offer examples of how a symmetrical approach to networks can be pursued, highlighting many of the material and nonhuman aspects in information processing. In the following chapter I outline the methodological approach provided by ANT, and describe how it has been adapted for this study. 


\section{Chapter: Expanding Translation as a Method for Techno-}

\section{Messes}

\subsection{Translation Devices and Distributions}

This section proposes to develop the ANT conception of agency, and its related notion of technological development, in terms of the concept/notion of translation. First, I examine translation as a guiding principle in enacting an ANT study, before exploring the ways this has been interpreted to guide the choice of specific objects of study and research methods. I then outline the ways in which I used this approach to engage with the human and nonhuman actors found in the quantum computing actor-network I examine. Translation offers a methodological framework to investigate how changes in the "regime of computation" (Hayles 2005) happen, and what these changes require of the broader social world. Engaging with computers through this approach shifts questions away from what a particular computing product determines in the world, and toward what needs to be determined to materialize a working machine. Translation provides a framework for mapping the development of quantum computing through the placements and trajectories of its problems, people, organizations, and materials. Following each of these allows us to better understand the breadth of computational activities, how computing works today, and how new possibilities come into being. 
The development of quantum computing techniques upturn many familiar characterizations about computers, making it an ideal moment to assess their social and technical positioning with the perspective of translation and the actornetwork. Translation appears as a foundational concept in what would only later be described as Actor-Network Theory; establishing a focus in ANT on how networks expand and distribute across new actors (Shiga 2006). It is an attempt to depict how relationships and meaning are created by the combined efforts and interactions of humans and other "nonhuman" entities (Murdoch 1997, Pinch 2010). Translation considers how some social actors become able to speak for and shape others as they are linked together. In this way, translation is at its core a question of how power is created, with an understanding of power as an emergent effect contingent on negotiations by many different participants that establish their roles and abilities. This differs from a view of power that depicts it as a resource or independent force that can be stored up or put to work to create structures (Law 1991, 1992; Latour 2005). Following the process of translation provides a description of the mechanisms of creating and maintaining power, showing how actors come into existence and are put into a social role.

As translation became a popular concept it expanded in several directions. I combine these here and propose translation as three related dimensions of analysis. First, the movement and modification of information from one form to another (e.g. phenomena to scientific fact). Second, the delegation of social acts/agency into new technology (e.g. installing an automatic door). The 
delegation of nonhuman technical components into human-enacted social acts is certainly a possibility, but rarely seen outside of temporary infrastructural failure or breakdown. The durability and reliability of nonhumans is typically greater (Latour 1992). Third, translation describes the process of creating a voice for designated actors, spokespersons, and roles for others. It describes a process of creating order and identity in the socio-technical network, an 'existence politics'.

Descriptions of translation almost always explain the process as a "transformation" to highlight that even though some essence is propagated, it is fundamentally a process of changing one thing into another (Latour 2005). However, just saying something changes isn't very revealing. Translation's strength as a mode of analysis is in bringing the mechanics and tactics to light of how a particular entity can be made to maintain some important qualities, while altering or discarding others, as the process attempts to create a desired social effect in a network. The desired effect may be created, or the desire may also be adapted during the translation process.

Translation studies sometimes take an inverted approach, following what happens when the transformation/translation goes wrong and a system falls apart. Translation studies consider how relations between the objects in a network are described, attempted, constructed, and whether they fail or succeed. From its first uses, translation has been elaborated several times in different ways to highlight complementing aspects of this dynamic. In place of translation, Latour has also used mediations, hybridizations, circulations, or associations to 
describe the "dynamic relations" and processes of actor-networks (Blok and Jensen 2011).To understand the different veins of translation in ANT literature, and how they complement in a way quite useful to communication and media studies, it helps to first briefly trace the concept's heritage.

\subsubsection{From Language to Order}

Translation rests upon an understanding of relationality developed by Saussure. He was interested in relationality as a framework for understanding how words and other signs connect to each other and come to represent their independent references. Saussure recognized words as products of social interaction that helped constitute the reality of human experience, and that, fundamentally, meaning was made by establishing a web of relations between the components of a system (Harris 1988). Saussure's semiotics is relational, in that the meaning of words is found not in an essential real-world referent, but only in relation to other words. No word is an island. This relationality is adapted in the translation approach with a much broader agenda than language. Translation in ANT is often cited as a "material semiotics" or "radical relationality" (Law 1992). The approach is 'radical' in that it applies relationality among entities across any set of types, not just between alike entities that easily appear as a sort of identifiable unit in a system. Most notably, translation attempts to include relations between humans and the wide range of nonhumans (i.e. materials, animals, organizations, symbols, etc.), rather than see these as autonomous categories of meaning or action. This extends the applications of relationality that have been used to deconstruct more narrowly defined systems, such as in Saussure's 
context of language, Levi-Strauss' of culture, or Luhmann's of communication (Farias and Bender 2009, 3). Anything that can be made to hold an identity and 'act' upon other entities factors into this extended semiotics. To emphasize this extension, ANT studies refer to their subjects existing in "heterogeneous" networks, actors, relations and so on (Callon 1981, Latour 1987, Law 1994).

This heterogeneity posits a socio-technical worldview that understands its subjects as unavoidably hybrid. Any social thing has aspects we call technical, and anything technical requires acts we call social. Whatever process or ordering we choose to study is going to be a hybrid of the two upon closer examination, rather than either purely technical or social. Translation's emphasis on heterogeneity aims to challenge a tendency to study alike-actors sorted by domains of study with presumed structural forces that shape their interaction. Instead, it favours attention to any strongly demonstrated association regardless of the types of actors involved, and relishes in the description of hybrid associations that break dualistic metaphysical assumptions.

The actors (i.e. humans, organizations, and other entities who can "speak" in some way) and actants (i.e. nonhumans actors who are spoken for/represented) needed to make social life happen, are similarly mutually defined by their network of relationships to one another. Translations' relational ontology of "mutual coproduction" (Farias and Bender 2009) is not a conception of relativism where anything goes. The character of an actor cannot be determined by the will of another actor or remade arbitrarily by a randomly connected network. As Latour 
paraphrases Deleuze, relationality is about showing how things are built, work, and gain meaning, not "the relativity of truth," but "the truth of relations" (Latour 2005).

The term translation itself is borrowed from Michel Serres, a frequent source of inspiration in ANT, especially in Latour's earlier work $(1985,1988)$. Serres used the term to describe instances of mediation where a communication process both transmits and distorts a signal. This distortion was dismissed or problematized by early models of communication that described it as "noise" or the limitation of an otherwise pure signal (Shannon 1948). For Serres, this impurity adds meaning rather than being a subtractive process. Serres wanted to imply a movement, displacement or infidelity in a communication that still performs a productive social effect. ANT borrows this attention to the added meaning from alterations that occur when something moves through the world, whether that thing is information, a behaviour, an identity, or other propagated actor. Translation accounts for the way actors interpret and otherwise relate themselves to the interests of other participants in the fact-building process (Blok and Jensen 2011, $39-40)$.

This conception of translation was first used to explain the construction of scientific facts and describe particular occupational techniques that occur in laboratories and scientific practice (Callon 1981, Latour 1985, 1987). Studies by Latour, Callon, and Law described the transformation of particular subjects of scientific study first into data sets via laboratory equipment, then into the visual 
media of test results, and finally, though gradually, into languages, scientific laws, and sometimes technologies. Their methodology suggested that science and technology needed to be studied in-action, and this was done by highlighting interactions between scientists, their tools, and their products. Increasingly, these translation studies followed scientists out of the laboratory, as they interacted with the broader world (Callon 1986a, Latour 1988). In every step of scientific practice, ANT studies observed a transformation that drastically changes the medium, and sometimes the content, of the information being translated, yet consistency is also maintained. The new translations corresponded well enough with one another to support a narrative provided by scientists.

The ability to closely resemble previous actors, but support the creation of a new narrative with broad social consequences, became a focal point of translationbased studies. They implied that the results of propagating networks of social action and meaning is deeply connected to the techniques/materials used to distribute the network. ANT writing argued that scientists have unique tools, but they do the same sort of world-building as everyone else (Law and Hassard 1999). In these early accounts of translation, science is given as one, very effective, method of creating a "chain" of linkages that enable some humans to speak on behalf of specific nonhumans or "nature" generally (Blok and Jensen 2011, 76). The linguistic analogy can be a little deceiving here, as to "speak for" does not limit itself to verbally describing what an actor does, but to be able to also persuade the actor to act that way. Callon's pieces on an attempt to build an electric car infrastructure in France $(1981,1986 b)$, and the manipulation of 
scallops (1986a), considered how a variety of nonhuman actors are translated to participate in larger networks of work by deploying many different enrollment strategies. These key studies became foundational and highly cited examples of ANT scholarship both within Science and Technology Studies (STS) and other fields flirting with the idea of nonhuman/human symmetrical study. They emphasize that seemingly passive actors/actants need to be convinced or accommodated in what can be very demanding ways. Like many ANT studies, these two cases highlight the collapse of a 'network of work' when an actor refuses to stay to the script provided for it by spokespersons.

Those spokespersons may be scientists, politicians, businessmen or others with established narratives of how those they describe a network working, but ANT studies often seem to rejoice in the rebellion of uncompromising actors and the difficulty that spokespersons encounter. Translations in these accounts are about establishing a worldview that assigns identities, roles, and expectations for each actor involved. Similar to Latour's often cited account of Pasteur and the adoption of vaccination in France (1988), Callon points to the seemingly empowered actors in these networks who become "obligatory points of passage" (1986a). Actors who function as points of passage are able to shape other actors in a way that is beneficial to them; they may become such things as arbiters of knowledge in scientific communities or patent holders. The concept indicates they have gained support for a vision of a network that requires them to work. These privileged positions are precariously based on the cooperation of many other actants. 
Translation then, is about the mobilization of human and nonhuman resources to propose and maintain some kind of order. First applied to understand the construction of facts and knowledge, Latour's focus shifted to the construction of simple mechanical artifacts that humans interact with in order to develop ANT concepts (e.g. handaxes, hammers, seatbelts, speed bumps). Later, ANT studies started to consider larger and more complex technical examples, such as mass transportation (Latour 1993) or a space shuttle launch (Latour 2010).

\subsubsection{Unfolding Nonhumans}

Translation is often deployed to highlight the useful and necessary work that nonhumans do that maintains social life. Though material objects can be translated in diverse ways, they cannot be constructed, literally and figuratively, to do whatever human actors demand. Latour describes an automatic door opener as having gone "on strike" to show that when a technology is broken it begins to reveal what social roles it has been delegated (Bijker and Law 1992, 225-229). At other times, he points to the disciplining, assisting, and extending that nonhumans (i.e. actants, artifacts, agents) do as they act on humans and each other to make the social world durable (Law 1991, Law and Hassard 1999). Behaviours maintained through human social actions can be translated, at least partly, into constructed material conditions. These materializations are not outside of or simply used by the social world, they are its integral constituents. For Latour, the translation techniques which create these social constituents are what make it possible to maintain the scale of social relations and divisions of 
labour fundamental to human society. For example, the translation of the humans who were employed as computers in the 1900s into digital computation devices, not only delegated previously human work to machines, but expanded the applications and performance of computation practices dramatically. Often this aspect of translation emphasizes moments of automation or technical improvement. However, the fundamental aspect is a shift of responsibilities and its consequences, regardless of notions of progress. Nonhuman computers both took over certain actions and performed new ones, changing the boundaries of computation and resulting in a multitude of new social relations.

In translation studies, power often appears to be exercised upon nonhuman entities, since they can be convinced by accommodation, engineering, or other methods to cooperate with social narratives. This also implies they are potential sites of resistance to proposed power structures/programs of action, which runs counter to common notions of agency in social science and critical theory as a human quality (Shiga 2006, 47). Considering nonhumans as equally consequential actors in society, even if we maintain an ontological or moral distinction between humans and nonhumans, has been a defining feature of translation studies. The expectation to treat these categories the same in analysis, that translation includes humans and nonhumans, and that nonhumans have agencies that enable them to act, is described as "generalized symmetry" (Law 1991). This symmetrical treatment of human and nonhuman agencies has led to several criticisms of ANT rooted in a misunderstanding that this was a claim of complete equivalence between humans and nonhumans. Rather than 
fully rehearse the "can objects have agency?" debate, it is valuable to turn to Shiga's (2006) consideration of translation in ANT, and how it shapes our perspective of agency away from an essentialist subject/object discussion. Shiga notes that studying humans and nonhumans symmetrically, and keeping translation as the core of ANT, focuses on agency as a distribution. If agency is distributed as part of a planned network, rather than something essential to one kind of entity or another, it is easier not to confuse agency and intentionality. Shiga describes translation as the means of distributing agency in social life; the question is not whether it is possessed by a certain actor, but by what techniques it has been concentrated, spread, or otherwise allocated. Shiga explains how regarding translation this way is central to ANTs conception of agency, "when divorced from the concept of translation, the actor-network becomes at best a synonym for other kinds of social, technical, or communication networks and at worst a restatement of the agency-structure debate" (40). Shiga highlights a passage of Latour's to finalize the point: "The name of the game is not to extend subjectivity to things, to treat humans like objects, to take machines for social actors, but to avoid using the subject object distinction at all in order to talk about the folding of humans and nonhumans. What the new picture seeks to capture are the moves by which any given collective extends its social fabric to other entities" (1996, 194, emph mine). Following this observation, Shiga argues that our "understanding of artifacts can be enriched by attending to the possibilities of resistance that emerge from the representation and imposition of identities on entities that seem merely technical" (43). 
An analytic framework based on translation examines the design of digital artifacts as the exchange of actions, skills, goals, and capacities between human and nonhuman entities. Approaching a socio-technical context with translation is an attempt to follow the step by step practices that entangle and hybridize what is usually presented as separate: the natural, social, political, technical etc. Law's early summary still holds:

The thrust of ANT case studies is this: the politics of artifacts should be reconceptualized to account for the actions that are routinely distributed or shifted between humans and nonhumans in the practices of scientists, technologists, and users. Each of these heterogeneous engineers acquires allies and draws power from them by transforming 'natural' differences between humans and nonhumans into distributions. (Law 1991)

\subsubsection{Following to Durability}

Translation studies came out of small-scale research around places where science and technology is practiced and designed. After entering these spaces, the most common methodological invocation from ANT is to "follow the actors" (Law and Hassard 1999, Latour 1996, Latour 2005). This can be problematic as an actor-network is not a clear cut entity but something partly generated by the researcher's summary (Law and Hassard 1999, Latour 2005). The boundary of describing an actor-network is dependent on the goals of the description, and the translations that form actors can become a kaleidoscope of endlessly shifting 
relationships. What following the actors has meant when presented with a potentially endless array of entities, is that the idea of the objective observer becomes impossible to maintain.

Downey (1998) outlines an approach with this in mind in his own study of computer engineers, "the main authority and responsibility for theoretical change lies beyond the researcher," being in the field is a constant process of "studying up' yet with responsibilities and commitments similar to those in good pedagogy...an effort at critical participation" (xi, 1998). He suggests the academic theorist "can advocate specific directions but does not possess ultimate authority" (xi). Instead, they are to learn how the boundaries between humans and machines are drawn from the everyday-engineering practices that inscribe them (27-29). ANT case studies tend to privilege or at least start with the designers, engineers, or other visible spokespersons of a technology to follow in the construction process. Most studies employing ANT concepts are modeled on this approach. However, much of Latour's later writing reads more as a reverseengineering of the translations of technical objects (Latour 2010, Hache and Latour 2010). Though ANT concepts and arguments have filtered out of STS to a host of different disciplines and applications (see: Sommerville 1999, Farias and Bender 2009, Rumming 2009), they continue to centre around questions of technology's ability to act socially. Particular tactics of the ethic or directive to be following and listening to actors have intentionally not formalized, and vary depending on the context of study. 
Described broadly as an ethnographic approach, qualitative fieldwork and interviews are common (see: Latour 1987, Hardie and MacKenzie 2006, Vannini 2009, Venturini 2010). Despite a frequent refrain of ANT being a method rather than theory (Law and Hassard 1999), the methodology is more ontological than practical. Translation shapes a historical description of a case study by following the iterations and mediations of ideas through forms, people, or machines as they are put to work, and considers what has been done to accomplish them. This is quite different from a static view of an organization or actor focused on its current structure or outcomes. Translation studies can serve to counter an assumed discrete (by type) or functionalist description of a network, and bring attention back to how it is that objects are brought into existence out of a hybrid, somewhat unpredictable, messy process:

This, then, is the core of the actor-network approach: a concern with how actors and organisations mobilise, juxtapose and hold together the bits and pieces out of which they are composed; how they are sometimes able to prevent those bits and pieces from following their own inclinations and making off; and how they manage, as a result, to conceal for a time the process of translation itself and so turn a network from a heterogeneous set of bits and pieces each with its own inclinations, into something that passes as a punctualised actor. (Law 1992, 6)

Translation taken as a process of 'passing' highlights how much work it takes to bring an entity into being, and how all existences are contingent upon relations 
that may change. The result of successful translation is somewhat contradictory, as the process involves creating numerous relationships and connections in order to produce an "aesthetic of autonomy" (Vannini 2009 34, Latour 1985). This autonomy is characterized in the literature by the appearance of durability, predictability, or stability (Law 1992, Farinas 2009, Latour 2010), where actors can be trusted to behave a certain way, and therefore to be, to a certain extent, ignored. When the configurations of an actor-network are able to be taken for granted they are referred to as "blackboxes", following the engineering nomenclature for a system that can be treated as an input-output device. The blackbox has a similar implication as writing focused on the naturalization of everyday or ubiquitous technology, where technologies become increasingly socially powerful as they become invisible (see: Bell and Dourish 2006, Hooft and Swan 207, Shirky 2008).

Ultimately, actors in ANT's perspective are really concealed networks of human and nonhuman elements. The ability to blackbox an actor-network, or to claim that its negotiations have become stable enough to assume their inner workings without constant interrogation, has frequently been necessary for progress in science, engineering, and other fields (Sismondo 2010, 85). However, translation studies show that no actor-network is completely stable. Though certain technologies, social relations, or material artifacts may become so ubiquitous as to seem permanent, irreversibility is only ultimately by degree. Blackboxes can be broken, opened, and reshaped when new research or controversy instigates a demand for it. When an actor breaks down, or its actions become unpredictable, 
then networks that have been blackboxed must be opened up. Or, an actornetwork may not quite manage to come together despite the best efforts of its most vocal actors; the blackbox may never appear and the relations fall apart, perhaps betrayed by pesky nonhumans (Callon 1987, 1987). These moments, where there is a failure to coalesce, are also moments that reveal translation techniques in mid-step. Translation studies try to dig up and understand these techniques, which become necessarily obscured by the autonomy of the new entities they create.

\subsection{Developing a Critical Cartography}

The process of putting ANT into practice as a practical method has been highly variable. For social scientists interested in following some actors, Venturini (2010) attempts to provide a thorough guideline for implementing ANT as a methodology by describing the exercise of a "cartography of controversies". The dynamic and ever shifting movement of translations can be a difficult process to study. It is not always clear where to begin. The metaphor of mapping is used to help initiate a translation study. It attempts to locate paths of translation for the researcher to approach, and to find ways to open objects into sets of relations and transformation. However, specific aspects of the cartography's structures may be abandoned after they have been explored in the context of a study. These are more suggestions than guidelines. 


\subsubsection{Observing Controversy}

Controversy has been a focal point for translation studies, particularly those which follow Latour's development of "political ecology" with its' close ties to climate change debates (2004). Drawing upon his experience as a teaching assistant for Latour, as well as his own studies, Venturini outlines how ANT scholars have taught others to investigate socio-technical phenomena. This cartography aims to outline "the practice of ANT unburdened of all theoretical subtleties" (258). Initially, students are instructed to examine a controversy, describing it with no particular set of concept or methods. Approaching a study as a cartography or mapping emphasizes a slow and hard process of initial familiarization and study of an area without a quick methodological shortcut (259). Venturini tackles the frequent Latourian guideline to "Just observe", noting it is quite different than a claim of unmediated observation. He makes three main points to address this, first: 'just' observing does not mean:

...that researchers are forbidden to employ pre-established theories and methodologies. On the contrary, not imposing any specific philosophy or procedures, the cartography of controversies invites scholars to use every observation tool at hand, as well as mixing them without restraint. At least at the beginning of their explorations, cartographers should make any effort to remain as open as possible. Surprise and curiosity should inspire their notions and protocols more than the other way around. (259) 
Second, he argues that researchers cannot pretend to be impartial through the use of theoretical or methodological guidelines. Objectivity is approached by multiplying the points of observation a researcher can make. Each point is created by engaging a research object with another method or from another point of view. This could include looking at different documentations of the same process, pursuing another line of questioning on a subject, or using another theoretical lens to evaluate a subject at another physical or organizational scale. Points can be created by switching such practices and perspectives. Description should be done by following as many investigative approaches as become available, rather than a consistent approach. This is a call for "methodological promiscuity" (260).

Third, Venturini restates Latour's notion of researcher humility, claiming that "participants to social phenomena may be as informed as investigators. After all, actors are constantly immersed in the issues that scholars contemplate for a limited time and from an external viewpoint"; researchers are reminded to "listen to actors' voices more than to your own presumptions" (260).

Following this advice, studies should start by finding places or objects of shared uncertainty that have relationships in flux. These moments are the inverse of ANT's descriptions of a blackbox. Venturini provides a simple definition for this state of controversy, "situations where actors disagree (or better, agree on their disagreement)" (261). As these moments unfold, researchers need to record what translation techniques are used to link previously separate actors and the 
hybridizations that result. Venturini describes the example of the climate change controversy, showing how it has linked very unlike actors (e.g. cars and glaciers). As allegiances and identities change in the example an ANT study is following, Venturini argues researchers need to resist an easy reduction or simplification. Instead, they should closely record new or previously remote actors as they enter into the shared world without a ready-made explanation for how this will happen. As new actors emerge, there is an onus on the researcher to acknowledge the inequalities created or preserved by the new actor-network, an aspect that ANT has at times been critiqued for lacking.

\subsubsection{Layers of Translation}

Venturini offers five starting layers of transition to analyze in a study. The first being statements to literatures. In this layer, there is a need to identify "the full extent of the controversial arena" by mapping a web of what is referenced in the statements that propagate it. This identifies what literatures are commonly used by those under study, including academic fields as well as other sites of discussion. The point is to find what separate discourses are now being entangled. The second, literature to actors. This examines how connections spread beyond the textual. Actors are often drawn in and made important where they were previously irrelevant due to new statements (e.g. "carbon emissions kill polar bears"). This draws attention to what new actors are brought in and what statements brought them. Third, actors to networks. Actors inter-act. Observing the tactics by which they create and dissolve connections among themselves is the main purpose of the cartography or study of actor-networks. Fourth, networks 
to cosmoses. This step seeks to contextualize the study as part of competing worldviews or ideologies. As a fifth layer, Venturini points out that common understandings of the world are not permanent, but temporarily assembled. A study may not treat all these layers as equally important, or work through them procedurally, but they prompt a study to consider a variety of avenues for analysis. These are helpful directions, but do not describe precise actions for all translation studies. They do suggest a variety of modes of translation to keep in mind. Below I describe the details of how this study was conducted with these directions in mind.

\subsection{Methods and Practice}

The following section describes how these methods were performed in the context of quantum computing, and concludes by considering how this approach is beneficial for studying technological innovations. Understanding the trajectory of quantum computing requires disclosing the variety of elements that shape its direction. To make sense of its current state of development, I needed to find as many ways to approach these actors and their translations as I could. At times, this meant talking to and observing human actors in different contexts and periods of time. It also required engaging with multiple literatures and sites of observation. The state of quantum computing could not be induced by looking at the existing history of quantum computing, the content of its academic courses, or its industrial aspirations and representations alone. Translation encouraged me to highlight the paths taken between such areas. This creates a focus on the 
relationships between the many unlike actors involved in quantum computing and what they have collectively produced.

\subsubsection{Research Subjects}

Being a reader of technology blogs, I had been generally aware of recent developments in quantum computing, and the existence of companies in Canada working on related projects. I initially engaged 1QBit through informal conversations with one of its co-founders in February of 2013 by email, and then over the phone. I had learned of their existence through a mutual friend, and approached them to discuss what predictions and assumptions the company had for the development of quantum computing. At the time, the company was in a planning stage, about to hire on its first cohort of researchers after securing initial funding and partnership agreements with the only existing commercial hardware developer, D-Wave Systems. At this point I was reading quantum computing textbooks and searching for news coverage of the industry through Factiva and other online search engines, in an effort to develop an initial reference point for these actors. After further conversations with the CEO, CFO, and another cofounder of 1QBit, in which I described the expected direction of my research, I was invited to their office and pursued a research agreement with the company through the Mitacs Accelerate program.

Mitacs is a not-for-profit organization that places academics in the private sector for collaborative and innovative projects. 1QBit had decided to grow their company substantially by using Mitacs placements, which are largely funded by 
the Canadian Government. They were open to allowing me access to the company through this program, in part because they were eager to get 'practice' setting up research internships. Mitacs holds default agreements regarding researcher rights and intellectual property with many Canadian universities, including Carleton University. This existing relationship created a simple mechanism to work around concerns about what I could eventually publish (i.e. no code or algorithms) and what rights I would have during the research program (i.e. no further company review or restrictions of my research). The Accelerate program allows placements for four month units, with a PhD student able to hold a maximum of six units. My initial agreement was for one unit, though this was periodically extended up to the full six units. Mitacs provided $1 \mathrm{QBit}$ with a way to create employment agreements with academic researchers that cut down on the company's own costs as well as provided a technique for compensating international students who would otherwise have limited private-sector options during their education in Canada. As will be discussed in Chapter 6, researcher positions at 1QBit were heavily dominated by such international students for a variety of reasons. As an early Mitacs candidate at 1QBit, my own placement was part of the corporate learning curve for exploiting this advantageous program. This included the company using my own experience in the program as a model for some of their other applications, and of Mitacs itself becoming a significant subject of my study.

On May 4th, 2013, I met 1QBit's co-founders in person to discuss the limits of my access to their daily operations. It was agreed I would have the same access as 
a management/operations employee, on the condition that I would not later publish or describe at an in-depth level proprietary code or algorithms. I was also asked not to name non-public research partners, though I could still describe them generally. This created at times humorous scenarios. For example, at the start of my research 1QBit and D-Wave were not public partners, leading to a situation where had I published in 2013 , I would have had to refer to "a commercial quantum computing company" generically when there was only one in existence. I saw these limits as extremely minor and not foreseeably impacting my research interests. Such broad access was allowed in part because of the executives' own interest in seeing their company documented and in the philosophy of science and technology. This helped make the company's staff available and at ease with my placement, who were otherwise not used to being the subjects of research.

I began conducting an ongoing survey of key terms related to quantum computing in the news media, especially the circulation of stories about 1QBit and D-Wave. These initially came out of this early reading but were expanded and reduced as new sources of terms became available by interacting with participants and reading new literatures. These included: D-Wave, qubit, 1QBit, adiabatic, quantum annealing, and quantum computer. During the first month of my Mitacs placement, I conducted keyword counts through the previous three years of Factiva's database to get a sense of when/how/where material was being produced about these companies and related technologies in popular press. From these I set up a shorter list of Google alerts to track how the 
dominant ones were used and spread during the rest of my research period. These were not dominant sources of information for the following chapters, but they are an example of attempting to gain as many points of observation on a network as possible. At times they provided my helpful references for quantum computing activity outside the company that I could then bring into discussion and interviews.

On May 16th, 2013, I began attending the office and observing the day to day operations of both the management/executive employees and the Mitacs-funded researchers. The first several months consisted of an orientation offered by $1 \mathrm{QBit}$ and representatives from D-Wave Systems. This orientation involved sessions similar to a series of graduate seminars, aimed at explaining the underlying technology at D-Wave, and the kinds of problems and industry partners 1QBit was initially interested in exploring. As researchers arrived at $1 Q B i t$ with a variety of academic backgrounds including quantum physics, electrical engineering, mathematics, operations, and computer science, many sessions began with high-level descriptions that allowed a researcher such as myself from the social sciences/humanities to start to grasp the work being proposed and the technological components involved. Along with a great deal of suggested reading from the researchers and company, this provided my initial basis for engaging with both the technology and its related fields. Much of this early reading provided starting points of translations undertaken by the researchers and described in the following chapters. These materials were combined into concepts that were hybridized with the researchers' fields/agencies, and 
transformed into new modes and technologies. Following this translation process took time and would have been nearly impossible had I come in at the end of the researchers own learning curve. Following the actors through these stages enabled my own understanding of what was being changed with each transformation, as well as see what actors were trying to retain and elicit in new translations.

I attended these sessions initially with a group of six new researchers. My participation in these early sessions was mainly passive, and confined to taking notes. During this time I was focused on observing the interactions of researchers and employees, the topics of conversation and study, their points of apparent difficulty, and the tactics they proposed and implemented for working through problems. Following these sessions I would frequently ask individuals to explain particular interactions I had not understood or because I wanted them to reflect on their motivations. For example, an early disagreement over terminology largely sidelined one seminar. I later followed up with the two sides debating the best relevant use of certain words. I wanted to understand what they perceived as being at stake with the definition, and to have them explain the tactics they tried to use to resolve the theoretical/terminological differences. These kinds of follow-up questions were largely after the fact and one-on-one with each research subject. This helped to track how a shared language was slowly established, and to differentiate how the researcher's fields influenced their perception of technical problems/limitations. In later seminars I was encouraged to participate more directly as these 'outsider' questions were seen by 
management as being productive for the group to hear to improve their communication. While I did do this on occasion, most of my discussions were with groups of one or two people and not in front of company management, and I was rarely willing to interrupt an ongoing discussion.

Several small mathematical and coding experiments began to occur at $1 \mathrm{QBit}$ after the first month of seminars, but distinct projects really started to emerge after approximately two months. As the seminars gave way to individual experimentation and then small-group projects, I took turns shadowing the teams that began to form around these early ideas. This involved listening in on brainstorming/planning discussions, continuing to ask clarification questions, and looking over the various kinds of documents they produced to develop and share their ideas. Common documentation included whiteboard drawings/notes, written mathematical theorems, flowcharts, pseudocode, code, and eventually research papers, white papers, patents, and PowerPoints/Keynotes. When these objects were digital, direct copies were provided to me. Physical items (i.e. notes, drawings) were scanned or photographed, and in some instances reproduced by hand. Conversations and meetings were recorded using the voice memo function on my personal iPhone, and then transferred to a laptop as well as a cloud storage service.

My analysis of this extensive documentation only focused in part on the content they represented. Just as important was using accompanying observations and questions to observe the function each document provided, the relationships they 
created between actors, the techniques used to create the documents, and the transformations of the content they require/allow. For example, researchers frequently drew out diagrams of the qubits described in Chapter 4 . These diagrams were drawn on the walls or glass doors of the office. Each drawing proposed different ways to group and connect qubits. Often these were drawn collaboratively during discussion, as different ideas were tried and erased. The logic behind a method of connection would occasionally seem promising and be left up. Then it would eventually be taken down in other formats, put into a textual-mathematical description, and coded into a programming language. While some of these qubit-connecting solutions were important in themselves, the redrawing process also reflected a growing flexibility and multiplicity around the concept of the qubit and how the researchers deployed it. It became grouped and given new drawn boundaries, and then circulated into other testable forms that required other competencies and people involved to mobilize as software.

Later in the company's growth, virtual meetings began to be common, especially company-wide Google hangouts. These were captured using that software's native recording options. However, I generally found my notes of such meetings more useful than the extensive hours of recordings produced. In cases such as the whiteboard drawing, some of these "documents" only existed beyond a few hours because of my intervention - either recording them or because the researchers 'shorthand' was elaborated on when I asked a question. However, many whiteboard elements did become long-lasting reminders and guidelines 
that stayed up for months on office walls and doors, sometimes because they were key insights, other times merely forgotten notes that other teams were reluctant to erase not knowing their importance.

As part of my research involved trying to understand the researchers' various projects, I began writing summaries of the emerging project goals and motivations in descending levels of technical complexity. This was often the result of extended questioning with researchers to be able to have them track back their technical problems to 'real-life' or higher level applications. These began as small conversations but evolved into impromptu extended interviews, though not to be confused with the series of planned interviews conducted later. In describing the technical projects and their ramifications, subjects often began long conversations about the valued products, currencies, and relationships in their academic backgrounds to help explain a project's significance, as well as to form their own reflections on the difference of conducting research in a privatesector environment. Longer conversations that began as a quick question often started in the office environment, but would transition to more informal settings, such as a cafe or neighbouring library. This was intentional, in part to encourage subjects to speak freely. Though, it was uncommon to get the impression that participants in this study were in any way reticent. They liked to talk about what they were doing and were opinionated.

At times my creation of project descriptions became part of the projects themselves. Approximately six months into this research, I was asked to assist 
management creating similar "layman" descriptions based of my own for the purpose of educating potential investors. At the outset of my research it was made clear that the company would only have full access to final publications resulting from my research and not my full research notes, including recordings or transcripts. The minor contributions I made to $1 \mathrm{QBit}$ 's documentations did not pose a challenge to this arrangement, yet it did require reflecting on the role of the researcher in translation studies. Chapter 6 describes the process of 1QBit translating academic researchers and disciplines into employees and resources for their network building. Instances such as the circulation of my descriptions indicate a parallel to Latour (2005) and Law's (2004) methodological arguments that the researcher constitutes the network they study. The network's translation processes also constitute me and my research within the network, incorporating my ethnographic methods to help further particular translations and refine the visions espoused by spokespersons. Quantum mechanics is well known for problematizing the notion of detached observation with the uncertainty principle and observer effects. Translation studies also cue us to not be naive about the process of following actors. This small example shows how the analysis/research process reifies into unexpected objects with unintended uses. These are not entirely within the control of an objective researcher or methodology, and reflect the agency of the actors being studied to look back at the process of their own observation.

Research and development at 1QBit was organized into terms to coincide with the academic calendar and the semester-based units of the Mitacs program. 
Following the first term the seminar-style of meetings became less prominent, largely because the known expertise had been disseminated and researchers were now investigating previously unresearched topics in quantum computing. At this point, researchers frequently had short bursts of break-out style meetings followed by individual or pair-based work. As their practices changed I shadowed them around the office and then followed up with questions on breaks, at lunch, or at times during their conversations. My questions at this point focused less on background information and more explicitly on their translation techniques. For example, how they went about identifying material actors they referred to (i.e. chose their words), put their representations to work in new contexts (i.e. interdisciplinary projects, new technology, partnerships), circulated immutable mobiles (i.e. produced documents and visualized data), created tasks for new technical actors and interpreted the requests and desires of the other involved individuals and organizations. As researchers began producing formal documentation of the projects, from summaries to white papers, as part of my internship I was asked to line-edit these documents as nearly all the researchers had English as a second language. This also provided me with an opportunity to have researchers clarify their individual work for me that I was not directly observing. Each researcher had a noticeably different idea about what it was they were innovating, often based on the academic fields they were trained. For example, while physicists tended to directly speak of quantum computation as the subject of their work, others understood their work as primarily about algorithm design or optimization techniques. 
After a year of project development and hiring, the company had somewhat stabilized in its growth, and I began scheduling formal interviews with researchers in June, 2014. At this point I felt well versed enough in the technology and business landscape to ask appropriate questions and appreciate the complexity of answers I expected I would receive. An additional factor in the timing of the interviews was to let team members have a substantial period conducting interdisciplinary research with each other, and interacting with the management and legal staff at the company, before I questioned them on their practices and terminology. I began interviews initially with three of the researchers from the first Mitacs cohort. Every 4 months, new cohorts were brought in to expand the team, or in some cases more frequently. As the company had very high retention rates, this meant the pool of available research subjects quickly grew, though not as exponentially as the qubit populations they worked to define and control, discussed in Chapter 4. During my main research period at $1 Q B i t$, the R\&D team grew from 5 positions to 28 . To reflect this growth, an additional 7 researchers, 3 executives and 2 operations employees also took part in solo interviews as my research period extended into a second year. Initial interviews with each subject were 2-3 hours long. All have had at least one 30-60 minute follow-up as new questions emerged in my research. These conversations have mostly been aimed at getting participants to be more reflexive about their everyday interactions with each other, their backgrounds, perspectives on the technology, and perception of the company and other organizations. As shadowing their everyday interactions gave me access to the 
technical details of projects, I wanted these interviews to be focused on what they saw as structural forces and to try to get the researchers to discuss the ontology behind their work. The researchers were aware of criticism about the 'reality' of work on AQC from some academic communities. They were not bothered by this controversy and did not consider it their role to resolve it. However, it motivated them to reflect on how they conducted their work, and how new technology and understandings were being created. They were often eager to compare and contrast their experiences in academia and industry. This helped me understand how they saw a bigger picture that the company was a part of, what was a mystery to them (e.g. investors), and gave them a chance to speak back to the assumptions they felt were in these structures. Conversations like these were not just tutoring sessions for me. They explained how the researchers became entangled in quantum computing and how they were being translated from graduate students to quantum software developers.

During the latter half of this first year, 1QBit began organizing and attending industry conferences. I was able to attend several of these, including those with D-Wave Systems, researchers from IBM, Microsoft, and NASA (see Chapter 6). These venues gave me the opportunity to interact with members of the broader communities involved in quantum computing, and see these organizational actors' interests from outside the internal discussions and perspectives of 1QBit. This was also a good opportunity for me to meet skeptics of 1 QBit and its approaches. 1QBit gave me permission to record these meetings, and this also let me interact with research partners and industry peers in formal question 
periods and informal conference-related settings. The majority of these were held in Vancouver, Toronto, and Mountain View, California. Vancouver conferences were also accompanied by tours of D-Wave's facilities in neighbouring Burnaby, BC. The D-Wave tours were welcome opportunities to get up close with the hardware itself, and ask questions with a variety of D-Wave personnel. I was given verbal permission to take pictures and recordings of D-Wave's operations, and received a variety of technical and promotional literature from the company. In total, I took 3 tours of D-Wave from August, 2013 to June 2014, and attended two press conferences at the same site.

In May, 2014, 1QBit opened a second office in Toronto, Ontario. Over the following year, which covered my remaining three Mitacs units, I spent most my time at this new location. While there, I followed the same patterns of interaction I had established in the Vancouver location. In a few cases, when I was in a different office shorter questions and follow ups were asked by email, Google Hangout or over Redbooth (a project management system used within the company at that time).

With new cohorts, especially at the second office, I tried to include researchers who would have different points of view, project topics, or academic backgrounds then the initial core group. It became clear quite early on that certain camps of thought existed between fields. The mathematicians and physicists, for example, had a (usually friendly) rivalry about how to conduct research, view technology, and what makes it important. This boiled down to what each considered the 
reality and usefulness of their disciplines research subjects. Engaging with researchers from new cohorts was important as the initial technical and expertise needs of the company did not reflect the direction and priorities of the research after an initial foundation was developed. For example, while physicists were extremely important in the early work done at the company, they were increasingly marginalized once they had successfully established understandable expectations and ideas around the computer hardware. Later chapters will examine this blackboxing process in detail. Once it had occurred, the insights of researchers from mathematics, operations, and software engineering became increasingly central to the core of research being prioritized by 1 QBit.

However, three initial interview participants out of the first cohort of six, were given the most time and follow-ups. During the research period they became the company's senior researchers, led team projects, and were adept at providing insightful descriptions of their work. These three were also utilized by the company on many occasions to be designated spokespersons for the overall research being done at a number of industry events and conferences. This made their contributions to my own research even more important as they began to occupy those leadership, organizational, and representational roles on top of conducting research. Their transition from academics to industry leaders became an important translation itself for my research, which was only made visible to study due to the extended period of my interaction with them. However, when using statements from interviews, observed meetings, conversations or shadowing, I avoided the assignment of researcher personas or pseudonyms 
unless I found it necessary to the agencies being described, such as distinguishing executives from researchers. While tempting to develop characters in this way, I wanted to work to equally emphasize the nonhumans in my description. Pseudonyms began to shift the account toward certain personalities and interpersonal relations. This undoubtedly limits the scope of my analysis, particular in discussing the different experiences of humans in the network. For example, like many accounts of technological spaces, the majority of participants were male, with approximately $1 / 3$ being female. Though women occupied technical, management, and operational roles, they were a minority. While gender did not play a blatant role in the developments this dissertation covers, it certainly affected who was likely to be in the room to observe in the first place. Similarly, while I do briefly address some of the impacts of the cultural and national diversity at $1 \mathrm{QBit}$, I do not go into detail about the ways these aligned with corporate hierarchy. For example, while white men were actually rare in the company overall, they made up $3 / 4$ of the co-founders. These realities speak to important broader issues in the composition of the technology industry and higher education, that I did not consider feasible to address in the scope of this study.

My list of interviewed subjects did not include many individuals with whom extended impromptu conversations occurred. These proved very informative on the same issues. Often these conversations were also recorded, and during the extended course of my field work, nearly all members of the company, and many in connected organizations, provided long statements or had question and 
answer sessions with me. This daily and persistent material gathering eventually outweighed the contributions from formal interviews. While I had initially intended to have the observation/shadowing period of the research inform the interviews, in practice it worked in both directions, with the interviews providing context for more productive impromptu questions and conversations while shadowing. The formal interviews also provided an unexpected function of increasing the researchers' interest in my own work. While all had been notified and accepted my presence at the company, the formality of declared interviews raised the visibility of my project even to those not interviewed. This created situations where I was occasionally approached by researchers at the company to have conversations about their work or explain my research. No negative concerns were expressed by the researchers about my work, and some were quite interested to know what a non-STEM doctoral project required.

While the majority of time in the first internship period was spent with researchers, a similar shadowing process began with the management of the company. I was asked early on to assist a foreign co-founder of the company with the grammar/spelling of some of his communications (e.g. outgoing email, project documents, grant applications). I attended management meetings which I observed to get an idea of the terminologies, concerns, and players relevant to the company's administration and business plan. Of particular interest were regular meetings where management would meet together with 1-4 researchers working on a project to get updates and make sure they were representing the project appropriately to outside parties. These meetings were often prime ground 
for managers and researchers to suggest possible applications of the researcher's early work, and as later chapters examine, debate the criteria for a project's value.

Because I attended these meetings and edited some documents for the company, I was given access to the online databases used by management to store all company documents. The majority of these were on Google Drive, for which I was assigned a company account. Some of these databases were open to researchers for their storage purposes, but others were restricted to management. These documents provided a rich source of background reading material to contextualize managerial actions. As a company literature, they complimented the academic literature compiled by researchers, providing important insight and context for observing people and places in person. They were also another medium for the actions I observed to be represented and circulated, and often important for enrolling outside actors in the growing network.

Researchers were mostly unaware of the business developments described by these documents unless they were announced by management at meetings. Management also routinely included me in meetings outside the office with a range of external parties, from investors to marketing and branding companies. I was generally silent at these meetings, but became an expected presence. Attending them was very useful, however, as it gave me the opportunity to follow how research documentation informed the company's representation. It allowed 
me to trace how project descriptions were being transformed for other purposes and directly ask why certain changes were being made.

Unlike the research team which grew dramatically, the management team remained relatively stable in size. Initially, management was comprised of the four co-founders who segmented their responsibilities. One of these co-founders moved on to a silent partner status during the first year of operations and two operations managers were hired as the company grew and moved toward a Series B investment round. Since I was "from academia" in this groups' eyes, and with no substantial business experience, ${ }^{5}$ this management group was quite eager to teach me their preferred terminology and about what they saw as relevant actors. In day-to-day conversations they described how they saw the business succeeding and how it would fit into a pre-existing landscape of technology and finance companies (i.e. who might hire it, acquire it, how, when, etc.). Their general assumption that I was a naive outsider to the world of business often put these conversations in an oddly pedagogical tone, where they endeavoured to teach me the right way to view the network. This helped inform my understanding of how they mapped and envisioned the network more than if they had just gone about their tasks. It also gave me the opportunity to compare their narratives to their actions. Over time my presence was treated in different ways. At one point it became clear that newer hires saw me as a co-worker or management assistant, even though they knew I was also doing some kind of

\footnotetext{
${ }^{5}$ That I had government work on my resume only horrified them further.
} 
research. That assumption made sense given that most of the researchers were also doing different research for $1 \mathrm{QBit}$ than they were for their own $\mathrm{PhD}$ requirements.

Interviews with management tended to focus on the motivations they had for working with this technology rather than for more established companies, and exploring their understanding of actors presented to them via researchers (e.g. qubits, algorithms, functions). 1QBit's CEO especially made himself available to answer questions about the company, including after my Mitacs placement was complete. This gave me a chance to quickly get comments or updates as major events for the industry or 1QBit occurred for which I was not present. These include their participants abroad at events held by the World Economic forum and European research agencies.

I heavily relied upon recording interviews, conversations, and meetings to avoid interrupting the flow of all these discussions (whether I was directly involved or not). Recording was simple and unobtrusive by using a smartphone rather than a dedicated recording device; however this greatly increased the time required for transcription and analysis due to the large library of recordings created. To cut down on this process, I highlighted what I considered particularly valuable recordings as they were backed up to the hard drive, and used information tabs available for voice memo files as they are imported to iTunes to embed brief annotations. 
Reading has been a major part of my research. The literature around quantum computation involves many fields, including those that have not been considered relevant to quantum computing until the work I studied at $1 \mathrm{QBit}$ took place. One of the less obvious things $1 \mathrm{QBit}$ produces is a new literature for quantum computing as it brings together the necessary resources for its projects. I have kept tabs on how this literature is created through the combined contributions (i.e. finding and writing articles) of the various research teams involved. 1QBit researchers have helped me grasp these materials as best I can, and in their own projects catalog where this literature comes from and seems to be headed. For example, the popular arXiv.org database run by Cornell University Library, quickly became a go-to source and destination for literature for the company, as well as other academics commenting on the industry's prospects. While 1QBit does keep a database of important literature for researchers, it has also been important to consistently ask individuals what they are reading, as many are also supplementing their work with literature from other and unexpected fields (e.g. molecular chemistry).

\subsubsection{Selection Criteria}

With a two year placement at $1 \mathrm{QBit}$, there were many events and technological developments I documented that are not explicitly covered by this dissertation. Choosing other meetings or examples to detail would undoubtedly change my account of a quantum computing network. This is the challenge Law (2004) lays 
out in describing complex messy networks: that many stories can be told and each helps produce a different network.

Over the course of my placement, many problems were encountered and solved by those I was observing to make that network take the shape it did. In my daily observation, their problem-solving actions often guided my attempt to follow the actors. It would lead me backward to explore the causes of the problems so I could appreciate what was being worked on, and forward again to literally follow people, documents, and the performance of ideas and technologies to see how these problems resolved. In some cases, over time, approaches to problemsolving became engrained as highly influential actants in their own right (see Chapter 5). As I followed the flow of translation that centered around certain problems, I used the sensibilities provided by the above cartography to alert me to the many nonhuman and nonspeaking actors involved, and how they were being transformed. This creates a preoccupation with including a heterogeneity of actors, to give fair and democratic representation to the nonhuman (Latour 1999). The network of quantum computing was also characterized by growth, and I wanted to communicate the ongoing enrollment and construction processes that reflected its expanding reach. However, this needed to be balanced against an impulse to assume this growth would continue in a predictable way or fetishize early signs of corporate success. 
Quantum computing is inherently compared and defined against the digital. As will be seen, its understandings and technologies exist in relation to digital concepts and their industrial legacies. My account, then, would need to address the defining frameworks of digital computation and development: hardware, software, and institutions. This was especially required since quantum computing deeply complicated these very divisions. While many examples from my fieldwork could have been chosen to speak to each of these, the actions and dialogues I observed highlighted certain actors. For example, the human actors frequently made comparisons with certain digital actors or processes that drew out tensions with these categories. Qubits were 'like CPUs...but not really'. The researchers were 'like software programmers...but not really'. They too suspected that quantum computing was put together somewhat differently, but were less interested in articulating how beyond technical systems. Their focus was on the daily problems that limited or stymied their computational visions. They were not at war with the material actants of quantum computing, but trying to get them to work. To play along with various creative ideas to make them compute.

These everyday negotiations, their resistances and successes, were incremental, and showed the ongoing change in expectations for quantum computing that typified the network. Focusing on examples that emphasize the recurring transformation in the network differs substantially from the view provided by the disruptive narrative described in the previous chapter. It also departs from the 
emphasis on controversy that brought the network to my attention, and for many on the periphery defined it. Choosing examples that highlighted quantum computing as a banal working-problem, rather than a mystical or exotic solution, appeared a way to make a meaningful contribution to the many descriptions of its existence. 


\section{Chapter: Gathering Distinctions for the Production of Qubits}

What is it that actually computes? Once the casings are stripped from the great variety of computational devices, what actant 'in there' is labouring away for us? The contents of the various objects we call computers have changed significantly in material, structure, and capability as they have been redesigned and engineered. The computer has taken on many shapes, with different ways of interacting with humans - their inputs and outputs. Changes in keyboards, screens, sizes of computer, centralized vs. distributed designs, or other aspects of the user interface have greatly changed how humans use computation and in turn how it uses us. As Edwards (1988) points out, these developments in computation have tended to be historicized into either a history of ideas that follows a progression of information concepts, symbols, and logic, or a history of calculating machines, whose progress creates new social and economic impacts. Edwards suggests a third approach, which follows computer development as a series of technological choices, which are made at many interwoven layers of relationships between people, organizations, and institutions. ${ }^{6}$ For all the variations present in modern computers, "the computer proper" is seen as rather consistent, a consistency that has in part allowed the superficial diversity of computers to arise (Feynman 1985). Looking at computers with this in mind

\footnotetext{
${ }^{6}$ Edwards aims to bring out the role of politics and culture in linking logics to devices, as a way to resolve a dichotomy in computer history based on software and hardware, and challenge notions of progress in favour of contingency and multiple determination. His focus on politics and ideology acknowledges the heterogeneous relations of computer networks, but tends to favour the human and organizational perspective.
} 
encourages us to momentarily ignore the input and output, and instead to examine what happens in the middle. Opening up and examining the pieces of this neglected middle is a favourite pastime of ANT (Bijker and Law 1992, Law and Hassard 1999, Berker 2006). The arrival of quantum computers has again made this necessary, as the fundamental building blocks of computation, both theoretical and material, can no longer be assumed and safely extrapolated into stable broader notions of the digital or computer. Or can they? This chapter examines how the rise of the binary transistor as a base for computation posits a historically and materially situated view of computation that differs in substance and logic from its quantum computing counterparts. The legacy of the binary transistor and the technological systems that have developed around it is far reaching and firmly ingrained in information processing. Altering it accompanies extensive changes to the theorization, techniques, goals and distributions of computation. This chapter shows some of the challenges and changes required to uproot the computer proper, and also how the binary's legacy carries forward into a new computational paradigm. Examining the base unit of computation, the bit, and how it changes in quantum computers, connects theories of information to material actants, and the agencies of material actants to the large sociotechnical networks that work to incorporate them.

There are many ways to situate quantum computing in relation with digital computing: logically, technically, materially, metaphorically, industrially, environmentally, and so on. Different actors in different contexts articulate the 
distinction between digital and quantum variously in trying to achieve different ends - sometimes competing, sometimes complementary, sometimes incommensurate. Academic discourse, journalism, industry promotion, training materials and even Canadian Prime Ministers activate different contrasts between the digital and the quantum and out of the cumulative relations of these accounts, qubits emerge, gain identity, and act. Gathering these explanations will take us through ways of contrasting bits and qubits as problem spaces, mathematical signifiers, materials, environments, and technological fabrications. Each of these bring the bit and qubit together, and separate them, in different ways. This gathering explores how quantum computing is "populated" as a matter of concern with many dimensions (Latour 2005b, 48). Bringing these together shows the many ways actors are relationally engaged to come into being, creating a cohesive socio-technical existence out previously separate discourses and acts.

\subsection{The Transistors' War}

World War II included computational goals that helped generate new technologies and techniques for computing (Edwards 1988, O'Regan 2008, Ceruzzi 2012). The canonical example of these is the work of Alan Turing, who established the model of binary information storage and manipulation as a method for general-use computing (Turing 1936, Dyson 2012). His approach laid the groundwork for all digital computers afterward. The devices Turing engineered were mainly aimed at specific goals, such as his famous decryption of the German Enigma coding machine. However, the binary system of 
computation Turing proposed inspired the design of machines that could more easily be put to a variety of computing tasks - making them "general purpose"7. The eponymous "Turing Machine" is a mathematical model that depicts how algorithms can be performed with a series of discrete symbols when provided with simple read/write instructions. It is usually illustrated as an arbitrarily long ticker tape on which binary values can be read and written from a control mechanism which can also move the tape (see Fig 1). The machine may refer to previous commands as it writes new ones, responding to the values on each section of tape in a linear process. By following simple boolean instructions in aggregate, complicated algorithms can be executed as programs.

A variety of materials could be used to enact a Turing Machine, and the different materials that have been employed produced different speeds of computation as well as different by-products, such as temperature, machine size, or cost of computing space. The development of electronic transistors in 1947 enabled incredible speedups of computation over other materials that had been used to create Turing Machines (e.g. punch cards, vacuum tubes) and have remained as the fundamental computing unit. Transistors switch electronic signals between states that can be interpreted as on or off, 0 or 1 . The molecular structure of materials called semiconductors, such as impure silicon, which are used as transistors, allows the flow of electrons between two points to be controlled by an

\footnotetext{
${ }^{7}$ Some aspects of the computer architecture usually referred to as "general purpose" were developed after Turing's' main contributions, such as von Neumann's designs for computer systems and chips and Shannon's information processing techniques.
} 


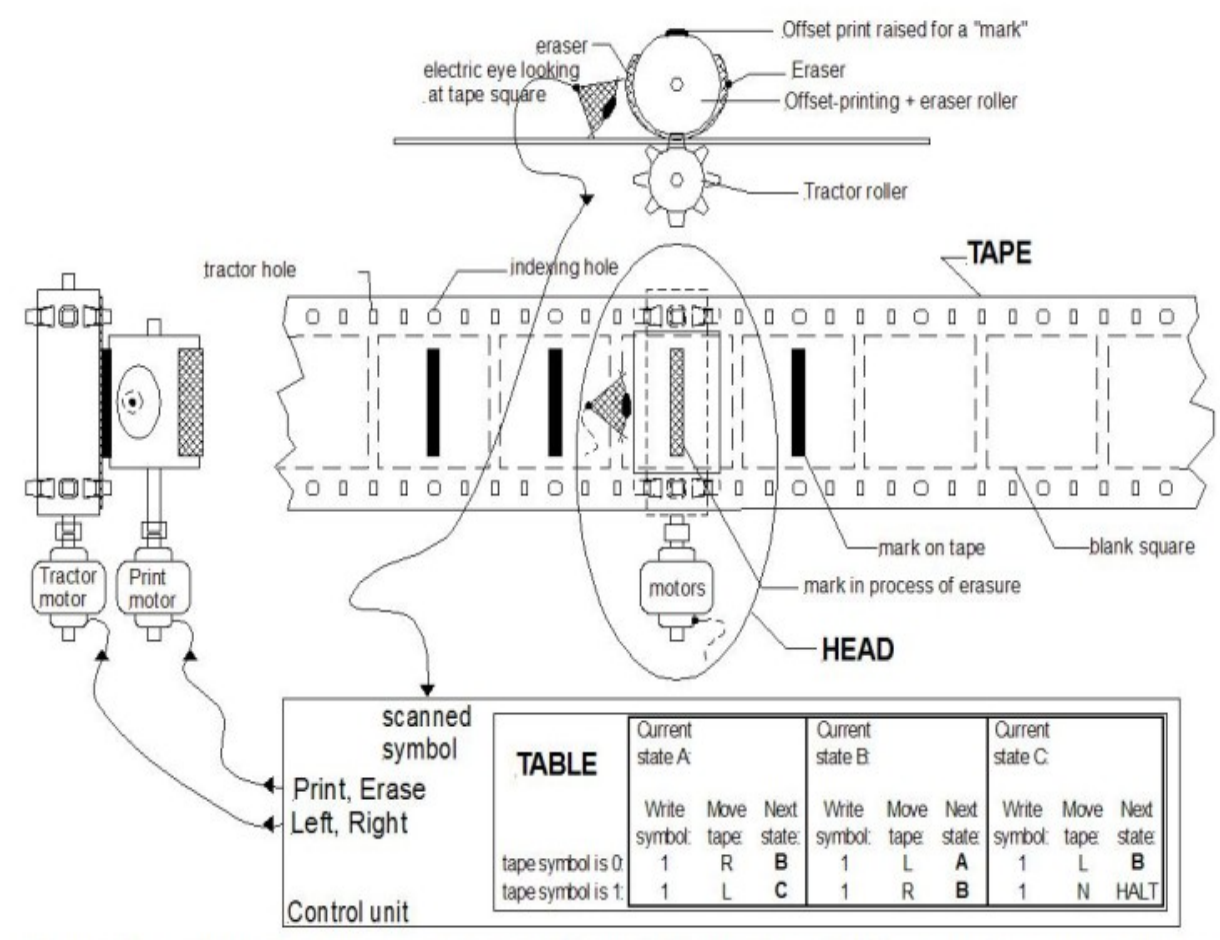

A fanciful mechanical Turing machine's TAPE and HEAD. The TABLE instructions might be on another "read only" tape, or perhaps on punch-cards. Usually a "finite state machine" is the model for the TABLE.

Figure 1: Source: Wikipedia. Turing Machine 1. User: Wvbailey.

electrode "gate" when a charge is applied. As manufacturing techniques of transistors have improved, usually making them smaller, digital computers are able to perform more complicated tasks faster, but the computation process has remained the same as Turing proposed it in 1936.

The Turing Machine operates on a simple system using binary sequences to represent and organize stored information. The simplicity of the system is made powerful because of the speed at which its many steps can be executed. This design favours a machine that is a "dumber but faster file clerk", capable of quickly looking up and reordering information (Feynman 1985). Along with 
Turing's decoding work, other high-powered calculation machines were being developed to assist in computation for other wartime projects that created a substantial imprint on computing technology (Edwards 1988, Ceruzzi 2012). Many of these projects developed techniques for information processing that would become useful as general computers capable of easily running many kinds of programs were built.

One such wartime project was the work being done at Los Alamos to complete reliable nuclear warheads. Nuclear explosions require carefully coordinated conventional explosions in the bomb that trigger the nuclear reaction. The calculations to plan these explosions required months of work, and Los Alamos had a dedicated IBM computational team for this purpose. They did not initially have digital computers, instead the "computers" of the time were teams of women working out the mathematics with individual desk mechanical calculators. Physicists Richard Feynman and Nicholas Metropolis had been brought onto the Los Alamos team to find a way to speed up this process. They organized a race between the humans with machine calculators versus newly procured IBM punched-card machines. The punched card machines were not as fast as those with electronic circuits but they demonstrated the ability of Turing machines to surpass both humans and mechanical calculators. Interestingly, the human-team at Los Alamos was able to briefly keep up by adopting a binary-inspired simplistic calculating system, but could not match the number of sequential calculations they could do without "getting tired" (Seidel 93). Humans make poor actants for enacting a binary system. 
As binary became delegated instead to various superior material actors, they enabled further information processing techniques that relied upon the step-bystep actions of binary processing and its sequential process of filing/refiling stored information. One such example is "pipelining", a technique developed by Feynman while working with the IBM machines at Los Alamos. Pipelining runs several calculations at the same time by staggering them in a carefully segmented sequence running through the binary processor. It was developed by realizing that the cards put into the machine could be divided and stacked in smaller more versatile series. This greatly reduces the time required for punchedcard calculations from several months to a couple weeks. Even though pipelining was created with cards in mind and based on their physical interactions, due to the inherent similarity of Turing Machines, electronic computers (i.e. those using transistors) were able to implement pipelining. Such transitions that feature the delegation of computing tasks from humans and mechanical calculators to diverse computational programmable machines and the information processing techniques they inspire, are familiar patterns of the new affordances granted by binary. Binary has been extremely successful in computing to the point it can be conflated with computing itself. This is especially the case when enacted by transistors. However, the limitations of binary, even when run at great speeds on electronic systems, have long been recognized. The postwar period saw consistent and staggering increases in computational power due to improvements in hardware. The observation of this hardware evolution has been colloquialized as Moore's Law, the now well known prediction that the number of 
transistors that fit on a single computer chip doubles every 18 months. This is usually reduced to the simpler comment that computer speed or power doubles every 2 years. This trend, while it lasted into the early 2000s (Pavicic 2006), has been a prime feature of predictions of near limitless computing power, resulting in optimistic expectations for areas such as machine intelligence and affordable computation (Kurzweil 1990).

Yet, in the midst of the transistor-led binary boom, physicists like Feynman were frustrated at the limitations of the digital approach to computing. From this frustration, an initial distinction between digital and quantum was articulated in terms of a problematic. Lecturing in the 1960s, Feynman explained that trying to model what goes on in the tiny regions of space described by quantum mechanics, takes a computing machine an "infinite number of logical operations to figure out". If a (near) infinite amount of logic is required to understand what was occurring in "one stinky tiny" area of space-time (Feynman 1961), binary computers would never get fast enough to work through them. At the same time, the more transistors improved the closer they were coming to a hard engineering limit (Pavicic 2006, Wu et al. 2012). When the ends of the terminals on each end of a transistor are too close, they can no longer distinguish separate off and on (0 or 1) states. At this too close distance, certain quantum effects would make what was an on/off-switch into an always-on non-switch. The pattern of creating more computation by shrinking transistors found a hard limit. Quantum effects limited the infinite progress of bit technology. 
Transistors are where practically all bits are born and housed. Their propagation, thanks largely due to their miniaturization, makes it practical to say that transistors are bits. Their prolific reproduction, with over a billion in a single iPhone, has made them the lynchpin of the digital age. As useful as punch-card calculators and other binary digit machines can be, it becomes materially implausible that "the digital" would have become the ubiquitous floating signifier of contemporary culture without the electric transistor-based embodiment. Unlike the nuclear reactions digital computers helped produce, transistor-based computation has become the fundamental building block of calculation, organization, and production in society. They are not, however, the only way of producing viable hardware for fast and complex computation. Replacing them as the fundamental building block of computation requires considerable changes to the theory and materiality of computation.

\subsection{Quantum Bits}

It was not until the 1970 s and 80 s that a major move toward quantum mechanics as a basis for designing computation began to take place. At this point researchers became increasingly interested in the creation and isolation of quantum systems for basic scientific research (Nielsen and Chuang 2011, xvii, 2, 6). After the 1970s, techniques were developed to "trap" and control single atoms, which slowly opened up approaches to use these as isolated and manipulatable quantum systems. Yet, it was not then an obvious idea to perform information processing using quantum systems. Studying quantum systems and applying quantum effects above the atomic scale did provide practical 
technological advances useful in understanding and improving objects like semiconductors that function in part due to quantum mechanical effects. However, the sampling techniques used to isolate and interact with quantum systems were very limited, and unable to control the interactions of several quantum elements at once. There was not a clear idea of what actual applications such research would even have as isolating quantum systems became possible (Nielsen and Chuang 2011 xvii-xx; Kaye, Laflamme and Mosca 2007). The idea of using isolated quantum systems to create a complete quantum computational device is generally attributed to Feynman's 1982 paper, Simulating Physics with Computers, though, the concept was quickly taken up and enriched by others in the physics community, perhaps most notably David Deutsch (1985).

Feynman's main motivation was resolving a problem regarding simulation because it restricted the further investigation of certain questions in physics. Physics describes a world that is continuous, but computers represent it with binary discrete values. Physics post-quantum mechanics also suggests that events are probabilistic from prior states, not deterministic as represented in a binary computer. Therefore, computers don't simulate physics, they "imitate" it (Feynman 1982, 469). Feynman wanted to consider whether "the possibility that there is to be an exact simulation, that the computer will do exactly the same as nature" (468), with the important caveat that "If doubling the volume of space and time means I'll need an exponentially larger computer, I consider that against the rules" (469). In other words, the computer can't scale as much as the problem 
scales. Computers at this point had shown the ability to imitate probabilistic outcomes for single quantum objects, but the resources required were untenable for larger calculations.

Computations based on quantum mechanics increasingly give "the most precise predictions in all of science", but applying quantum mechanics to some problems was becoming too complicated for classical computers to process (Kalai 2006, 14). Feynman claimed that classical computers would require an exponential growth to be able to complete quantum calculations, and that there are some calculations that classical computers simply cannot process or model. The best way to model a quantum mechanical system seemed to be with quantum mechanical parts. Feynman suggested a model based on the manipulation of photons and introduced the term "quantum computer", though many key elements of how that term is used today came later. These quantum mechanical parts became known as quantum bits or "qubits", and would differ in how they represent information. In Feynman's terms, this would fundamentally change our ability to simulate nature compared to a binary encoding.

At this point, qubit/quantum and bit/digital differ in their relationship to nature, insofar as "nature allows a quantum mechanically coherent superposition of 0 and 1" (Landauer 1996, 189). This means that qubits don't have to choose a single value because quantum phenomena don't only possess a single state or location. They can be represented as 0 or 1,0 and 1 , or values in between. This is useful for simulating the probability of quantum mechanics, and also for 
making any incredibly large set of calculations. Deustch (1985) noted that because quantum bits can hold more than one state at a time, if ordered correctly they could be made to run many calculations simultaneously, producing an answer based on all these "parallel trajectories" (Nielsen and Chuang 2011, 6).

Qubits would also be able to draw upon one another to decide what value they should have. Unlike the "file clerk" approach of bits in classical computers, where each bit holds an independent value that is stored until explicitly called upon, qubits are strongly influenced by each other. This type of influence is referred to as entanglement. Entanglement was famously described as "spooky action at a distance" by Einstein (Bell 2004, Halpern 2015, 137). As with many popular phrases of quantum theory, this was at first meant mockingly, but the phrase stuck as a catchy descriptor. Strange or quirky metaphors and analogies are a regular aspect of scientific creativity and as tools for education, often as ways to make strange objects more relatable and intuitive (Hallyn 2000). The sensibility of quantum behaviour is, however, completely unintuitive to brains grown in the classical world. Many of quantum mechanicals colloquialisms work to break our expectations from everyday life. Cats are either alive or dead, there really isn't a middle ground. Rather than making the quantum world relatable, they tend to remind us how unrelatable it is (Meglicki 2008). This sense of strangeness in popular descriptions of quantum mechanics is sometimes abused by guruphilosophers (e.g. Chopra 2015). Entanglement, though strange to human brains, is a fundamental aspect of quantum computers. 
Particles that are entangled can be separated by large distances and still retain a connection whereby influencing one produces effects on the other, despite lacking a clear causal connection. ${ }^{8}$ Entangling qubits with one another allows them to share information and calculate in more powerful ways. If you have a bit that can have superposition (multiple values) and entanglement (active relationships with other bits), then you probably have a qubit. However, though certain attributes such as these can be described as generic to a qubit, the development of various binary bits in different materials and machines greatly shapes their traits and usefulness.

The qubits this chapter examines in depth are substantially different in their materiality and computational abilities than other imagined qubits. However, they have the advantage of existing. Before considering their material particularities, it is useful to consider how qubits still relate to binary. Many of the distinctions gathered in this chapter consider the digital and quantum to be a binary. A binary binary! That the distinction is made is important, but it is part of a less clean relationality between digital computing practices and quantum computing's new novel actors.

\subsection{0 and 1}

Constructing computation around a qubit does not mean completely abandoning binary. Binary computation uses 1 and 0 to encode all values in bits. Similarly,

\footnotetext{
${ }^{8}$ More formally, entangled particles cannot have their quantum states described independently.
} 
quantum bits still use 1 and 0 . The difference in how these systems assign these two digits is in their determinacy. Digital systems require each value to be 1 or 0 , whereas, as explained above, quantum systems also allow for a superpositioned value of 1 and 0 . This 1 and 0 has become a common focal point for journalism covering the development of quantum computing, as well as for companies explaining the technology to clients/investors and other outside groups. 1 and 0 is presented as a powerful talking point because it is incompatible with the transistor, and therefore breaks the uniformity and expectations that the transistor propagated. There is a certain reverence for the qubit as it pulls off this seeming identity paradox. This likely results from the combined aura of quantum unintuitive strangeness and the somewhat blinding excitement that accompanies new technology product releases and investment (Cassidy 2003). During the Bloomberg Canada Economic Summit in 2014, D-Wave CEO Vern Brownell was interviewed by Hugo Miller, a technology reporter. Before asking Brownell several broad questions around investment strategies for the technology sector, Miller performed a typical hand-wavey preamble stating that he did not really understand superposition and "could not begin to". This apology included reference to the 0 and 1 issue, which Brownell briefly addressed by defining superposition and implying its calculative advantages. This produced much excitement and meaningful nods on stage and by those watching the panel. The CEO's response did not, however, add any more information than Miller's question, essentially acknowledging that the superposition state does exist. In other conversations Brownell has shown a deeper understanding of the 
technology. But what comes out in frequent public/journalistic discussions of superposition is this reaction of confusion that the short-hand for computation, 0 and 1, has been broken. ${ }^{9}$ It wasn't clear the Bloomberg audience or panel chair had a rigorous understanding of classical binary either, but the binary transistor is so normalized it doesn't create the same conceptual intimidation.

This " 0 and 1" ability is an important distinction but it is rarely truly examined in further detail to see how it creates significant differences from digital computation. Just as "bits" went through several materializations before becoming effectively synonymous with transistors, qubits have a number of different materials being explored for computational and architecture advantages. The particular qubits discussed here reside in D-Wave's Vesuvius machines, which were the most common quantum device used by 1 QBit during my fieldwork. These are an example of superconducting quantum computers, one of many possible quantum-based architectures for qubits. Qubits in a different quantum computational system could look and behave quite differently. These computers initially held 512 qubits, though as the technology progressed during my fieldwork the qubit population in each device grew up to 1024. Unless specified otherwise, references to quantum processors, quantum annealers, adiabatic quantum computers, or derivations of those refer to these machines.

\footnotetext{
${ }^{9}$ At a separate event, where 1 QBit was introduced to speak at an accelerator in Toronto, the director of the accelerator excitedly and confidently summarized quantum computing as unlike digital computation because "bits are always 1". Such mistakes reflect a widespread understanding of the normalcy of binary digits and the radical nature of the non-binary, even if shallow.
} 
The construction of this particular species of qubit as the basis of computation requires both theoretical and material manipulations of what composes a qubit. As this chapter shows, even though they are the fundamental building block of quantum computation, they exhibit an instability inherent to this rethink of computation, and require widespread accommodations throughout their sociotechnical network.

Digital computers take strings of bits as input, perform an arithmetic program on them, and output a new bit string. The power of the digital computer results from the speed at which simple mathematical operations can be performed on bit strings. This computation procedure has been extremely successful at performing many computational tasks. Quantum methods disregard this approach of transforming bit strings arithmetically based on stored information, so that they can solve problems unapproachable with digital binary devices. For example, in many kinds of optimization problems, adding more variables increases the difficulty to compute exponentially on a digital device, making them practically unsolvable (Rønnow et al. 2014). When inputting problem-data at a linear rate creates an exponential growth in computational difficulty, quantum computers have an inherent advantage over the digital. A useful illustration of why can be found in the "light-switch problem". In this scenario a set of light switches need to be configured to an ideal setting. For example, the setting in a real-life example may be finding what configuration of available lights should be used to completely light a stage while using the least amount of power. The stage may have many lights to choose to turn on and off, and some lights may 
use more power than others. To keep it simple let's say the switches are not dimmers, and can only be set to on or off. ${ }^{10}$ With 2 light switches it would be easy to check every possible setup exhaustively with a digital computer, or even on our fingers, since there are only 4 possible options for setting the lights. A digital computer could input each configuration, perform its arithmetic function, and check the resulting outputs to see which of the 4 is best by whatever criteria we decide to measure. With 10 switches there are now 1024 possible answers. Hard for a person perhaps, but still easy enough for a digital computer. However, 100 switches creates $1,267,650,600,228,229,401,496,703,205,376$ options! With a few hundred switches the time it takes to check each answer with a digital computer makes the exercise pointless, since it would require more time than is available; the sun will have collapsed long before you finish. Examples like these are used to reveal the scope of difference between digital and quantum regimes of computation. They introduce the significance of problem structures that scale in difficulty with the number of variables, and depict some methods of problem solving as impractical.

Light switches are a binary problem in that each variable has 2 states to choose from. But trying to solve it with a binary processor is incredibly difficult because of this scaling relationship, described by the simple term $2^{n}$. This example of the light switch problem is one that D-Wave tutorials use to teach programmers how

\footnotetext{
${ }^{10}$ This makes the problem discrete rather than continuous. Discrete optimization problems are very common in the real world, as they reflect decisions taken or not taken. To buy a sell a stock at any point in time is one example. A (single) stock cannot be partially sold.
} 
to think differently about using qubits instead of bits. ${ }^{11}$ To check the result of each of each light switch configuration, a digital computer would have to generate bit strings that reflect each switch in one of its possible positions at a time, try that combination, make a new series of bit strings that reflect the next combination, try that combination, and so on. By drawing upon the quantum mechanical phenomena of superposition and entanglement, quantum computers are able to take a different route to finding an answer to this kind of problem that has so many possible answers, or a large "solution space".

Qubits are able to use a physical process rather than an arithmetic one to approach such problems. Each light switch can be represented by a qubit, without deciding which configuration of on/off is being tested. With all the light switches set as on and off simultaneously, this quantum representation based on superposition must include the most optimal answer. In essence, the bet of whether any switch is on or off is hedged. The correct position is made to emerge by forcing the qubits to settle into a single, classical (i.e. not superpositioned), set of positions. This is done not by checking each one in turn as a digital computer would, but by physically assigning a "bias" of varying strength to each qubit with a magnetic field. The bias associated with each switch/qubit would reflect a part of the problem that complicates finding its solution. In this case, perhaps the

\footnotetext{
${ }^{11}$ The light switch problem was also a frequent example used by 1 QBit managers to explain exponential computation growth and optimization variables to investors or other partners without deep mathematical or computer science knowledge. It was deployed to impress as much as inform.
} 
energy consumption required per switch ${ }^{12}$ is created as an important influence on each of the qubits through a corresponding magnetic field. This representation of the forces at play on the switch are not computed in the same sense as the digital series of arithmetic, but instead the system naturally settles into a lowest possible energy state by the physical-energetic relationships of the qubits in the system and their surrounding forces. This use of natural interactions as a form for computation has been addressed by scholars who describe both ancient and modern analog computing methods, as well as the overlap of philosophy and computer science which posits that the natural world can be understood as a computer (Wolfram 2002, Lloyd 2006, Fuller 2008, Lloyd 2013). ${ }^{13}$ This moves computation from being a well defined set of calculative steps, to a process of carefully constructing relations between material objects, and deploying the outcome of that constructed relationality as a computational process.

\subsection{Peaks and Valleys}

The dominant metaphor used by $1 \mathrm{QBit}$ and $\mathrm{D}$-Wave in their documentation to describe this computational process is the notion of a landscape. It is not uncommon for mathematics and physics to use "landscape" for a large range of possible answers, and this has been particularly normalized in optimization

\footnotetext{
12 The lightswitch example is limited here, as such "bias" includes negative numbers, but as a rough analogy .

${ }^{13}$ This line of theorization branches out from the initially more specific (and prolific) work of modeling computation on human brains and their operation, from von Neumann's (1958) The Computer and the Brain onward to the recent surge in A.I., neural networks, and deep learning research (Buduma 2016).
} 
studies (Rutenbar 1989, Liang 1999, Leary 2000, Hansmann and Wille 2002). The landscape metaphor is used to create an intuitive understanding of complex phenomena, and helps create discrete versions of continuous problems with many possible solutions (Muller 2010, Linoff and Berry 2011). A 2D or 3D wireframe drawing is often used to represent all the possible answers to a problem, which is then searched (see Fig 2). The task of the qubits is to take the description of the problem, generate an unseen map of this landscape, and identify the lowest point on the map. This point reflects the optimal solution to the problem the landscape describes.

The landscape imaginary is a mainstay of classical optimization and digital 'search' techniques which have found ways to explore these computational territories, such as simulated annealing. ${ }^{14}$ When qubits are used, however, new techniques to search this landscape are available, which complicate and somewhat break the landscape metaphor. Simulated annealing is a well-known and popular search technique that involves checking randomized points on the

\footnotetext{
${ }^{14}$ The landscape concept traces back to early work in modern chemistry and (Eyring and Polanyi 1931) before becoming common in the study of Hamiltonian systems (i.e. quantum mechanics) and many other areas of study (Muller 2010).
} 


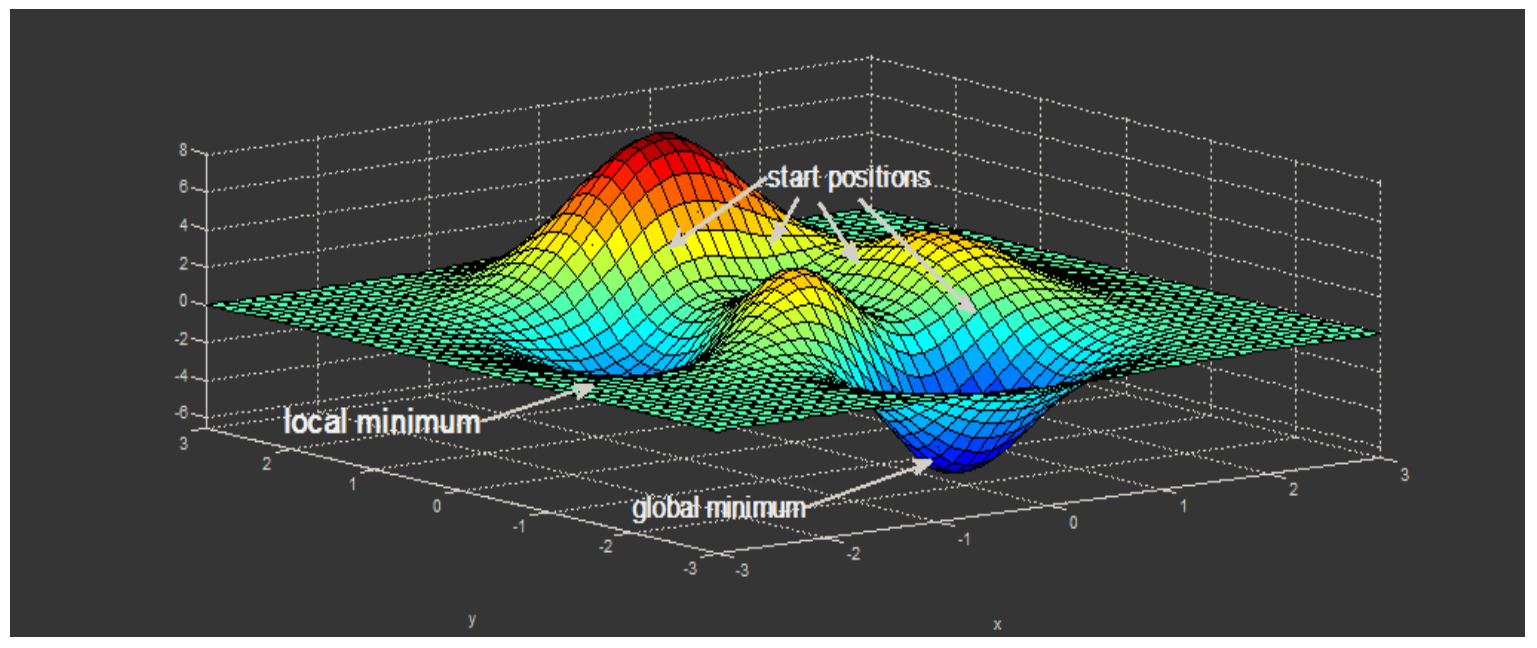

Figure 2: Energy landscape with local and global minima, and arbitrary start points for a search process. Source: Hmolpedia.

landscape and jumping around to new (pseudo)random points to see if they are a lower (i.e. better) or higher (i.e. worse) altitude (Cramas 2009, Ronaugh 2014). Eventually this jumping localizes on promisingly deep areas. Jumping works pretty well, but it creates a situation where the digital jumper may get stuck in a deep valley between two high mountains, and not have a chance to search for even deeper valleys (i.e. better solutions). Qubits can do more than just jump between points, they are described as being able to "tunnel" right through the landscape. D-Wave conceptualizes this by trying to add new layers to the landscape metaphor and its occupants:

The special properties of quantum physics, such as quantum tunneling, allow the quantum computer to explore this landscape in ways that never before have been possible with classical systems.

Quantum tunneling is like a layer of water that covers the entire 
landscape. As well as running over the surface, water can tunnel through the mountains as it looks for the lowest valley. The water is an analogy for the probability that a given solution will be returned. When the quantum computations occur, the 'water' (or probability) is pooled around the lowest valleys. The more water in a valley, the higher the probability of that solution being returned. A classical computer on the other hand, is like a single traveler exploring the surface of a landscape one point at a time. (D-Wave "2x", 3)

The digital here is strangely humanized as the logical cartographer, who can't compete with the elemental forces of quantum computation. Quantum annealing was proven to have a higher probability of an ideal result than simulated annealing for combinatorial optimization problems in the late 1990s (Kadowaki and Nishimori 1998), though it was purely an abstract theorization at the time. Quantum annealing was proposed as an improved method, though Nishimori later commented it was "crazy" that someone had manufactured a machine on the principles he outlined because of their extreme engineering requirements (Nishimori 2014). Annealing refers to the way physical systems, such as molecules, will reorder themselves after they are moved through an energy state. A common historical example of annealing is the process of rapid cooling blacksmiths applied to metal to create a new arrangement of molecules with new overall properties. Such classical annealing processes and quantum annealing are physically distinct, but quantum annealing is understood as metaphorically similar to the process of classical annealing. Putting a quantum system through a 
"schedule" of energy fluctuations continually lowers the amount of energy in that system and creates a new distribution of that energy landscape. Setting up and reading the changes in that landscape, a subset of the study of quantum annealing, is referred to as adiabatic quantum computation (AQC).

\subsection{Logic to Energy}

Instead of transforming determined bits with the logic program of a digital computer, qubits have undetermined states that are put through an "energy program". The energy program transitions quantum bits into the static values of a classical bit. When the computer creates these intelligible classical bits it does not return a single answer. Quantum computers' are probabilistic, which as a consequence means that the computer returns multiple answers. By comparing the answers the computer produced, the reliability of the answer (i.e. whether the computer got it right) becomes part of the solution. By asking the computer to answer the same problem hundreds or thousands of times, the consistency of the answer reflects the likelihood it is right. In this way, the uncertainty of the answer, which is a product of the uncertainty of the quantum states of each variable, becomes a central tactic in validating answers. This uncertainty can also be exploited in iterative computational processes, such as machine learning, where correct and incorrect answers are used to adapt the code of a program and subsequent computations. Since there is an array of solutions, even though the probabilistic qubit is still collapsed to a binary state by the end of the annealing/computation, it retains a distinct solution from a binary transistor attempting the same computation. 
That qubits produce this kind of answer lends them to certain applications. The light switch problem described above is borrowed from D-Wave's reading tutorial series aimed at clients for its devices. ${ }^{15}$ As they put it, "We didn't build a machine to play a strange masochistic light switch game" ("Quantum Computing Primer" sec 2.1). Instead, they identify a range of real-world problems, that at their heart, require finding optimal configurations of binary variables. These include image recognition and labeling, natural language recognition, or finding correlation in biometric data. In presentations to outside groups, they frequently highlight six market areas: defense, intelligence, cloud applications, national labs and universities, finance, and energy. Some of these applications and sectors will be explored in following chapters. The breadth of these proposed relevant areas suggests that even though specialized toward certain forms of problems, these qubits can somehow be made to align with many different actors. A qubit is, however, a difficult actant to produce and control.

\subsection{Accommodating Qubits}

Transistors switch electronic signals between relatively low or relatively high amounts of voltage that can be interpreted as on or off, 0 or 1 . Voltage is the digital's technique to encode information on transistors. Instead of voltage, the production and commercialization of quantum annealers is based on superconducting qubits that use magnetic fields to encode their qubits. The

\footnotetext{
${ }^{15} \mathrm{http} / / /$ www.dwavesys.com/tutorials/background-reading-series/quantum-computing-primer
} 
above descriptions of the qubit have been fairly immaterial, in that they describe the mathematical properties a qubit must be able to possess. The actual materials of constructed qubits are not independent of their actions as bits. Not only was the above abstraction of qubits based on decades of experiments with particular quantum mechanical objects, but the difficulty in creating and controlling realized qubits means accommodating materials in very extreme ways. Transistors have been materialized in substances like silicon and carbon nanotubes, but these materials did not shape the theory of computation that is applied to them, they just made it more practical. Binary can be enacted, if less effectively, in many other media. Techniques like quantum tunneling to improve combinatorial optimization, arise out of the physical properties of particular quantum mechanical systems rather than number theory.

Instead of silicon, the primary computing material for an annealing-based quantum computer is niobium. Niobium ${ }^{16}$ is a "soft, grey, ductile transition metal" that has a history of application in superconductors, welding, nuclear industries, electronics, optics, numismatics, and jewelry. ${ }^{17}$ The way it reacts to temperature motivates the material shift from silicon to niobium for the qubits' construction. When a low enough temperature is achieved, niobium acts as a superconductor and exhibits quantum mechanical effects that can be isolated and controlled as a qubit. Instead of using voltage to encode the physical bit, two magnetic fields around each niobium qubit can be measured to point or spin in one of two

\footnotetext{
${ }^{16}$ Atomic number 41 . Had it been 42 the Douglas Adams jokes would be insufferable.

${ }^{17}$ https://en.wikipedia.org/wiki/Niobium
} 
directions. These directions/spins are labeled as +1 or -1 to be turned into bits. ${ }^{18}$ When in a quantum state (during the annealing process) each qubit has not "decided" whether it is in a +1 or -1 position. Its current flows in both directions, causing magnetic fields also pointing in both directions. This is how niobium exhibits superposition. At the end of the annealing this dual-state collapses into a determined value. D-Wave and 1QBit characterize the qubit as having a kind of agency, describing it as "choosing" or "yet to choose" and "undecided" about what state it will occupy. The agency of qubits comes from their ability to act in decisive ways that will impact and direct other actors in the computing system. They can be put to work, but not entirely predicted. Theoretically, the qubit always chooses its reliable destiny of a position that results in the lowest energy state it can attain as part of its qubit community. How it achieves that state is not, however, entirely under the control of its programmers and can be disrupted by other actors in the network leading to errors. To be a qubit requires a great deal of support and a strange state of figurative free-will and yet mechanical hyperprecision. Qubits are a crucial point at which materials are distributed agency that enable the computation to occur, though as will be discussed below this ability to act is made possible by many other distributions and carefully balanced relations.

In order for quantum computation to take place, qubits have to be connected to each other as a multi-qubit processor. Connected, they can exchange information, influencing the final state each one assumes in the system. The

\footnotetext{
${ }^{18}$ The simultaneous use of $+1 /-1$ and $0 / 1$ to represent the state of a qubit seems to be the necessary baggage of asking physics and math to work together.
} 
information these states result in comes out of a relational system, where each qubit exerts agency through the energetic-informational exchanges of entanglement to co-produce each other's information values. Each niobium qubit is put into the shape of an outline of a long thin rectangle, and laid out in parallel or perpendicular to other qubits in a type of grid (see Fig 3). The metallic grid of qubits is able to connect and exchange information, so that each niobium piece can influence one another at the junction where the rectangles overlap thanks to similarly constructed couplers. "One can think of the qubits as being like neurons, and the couplers as being like synapses that control the flow of information between those neurons" (D-Wave "Quantum Hardware"). These connections allow for the niobium qubits to become entangled. However, this arrangement of strips of overlapping/connected niobium, places restrictions on the theoretical model of a qubit. The grid formulation is manufacturable, but only allows the niobium qubits to be connected directly to a limited number of other qubits in the system. That this chip architecture is therefore not "fully connected" has raised a long series of questions and at times harsh criticism from the physics community, primarily focused on whether this "sparse" series of connections really allows entanglement and therefore quantum computation. This doubt over whether the niobium really can be made into a qubit has been a source of controversy in the academic community. ${ }^{19}$ To a degree this has been resolved as doubts about the processor's existence were replaced with investigations of its efficacy, though

\footnotetext{
${ }^{19}$ A gathering point for this criticism was Scott Aaronson blog, Shtetl-Optimized. Aaronson was particularly vocal between 2007-2013, claiming the title of "Chief D-Wave Critic", before toning down his criticism in 2014. http://www.scottaaronson.com/blog/?s=d-wave
} 

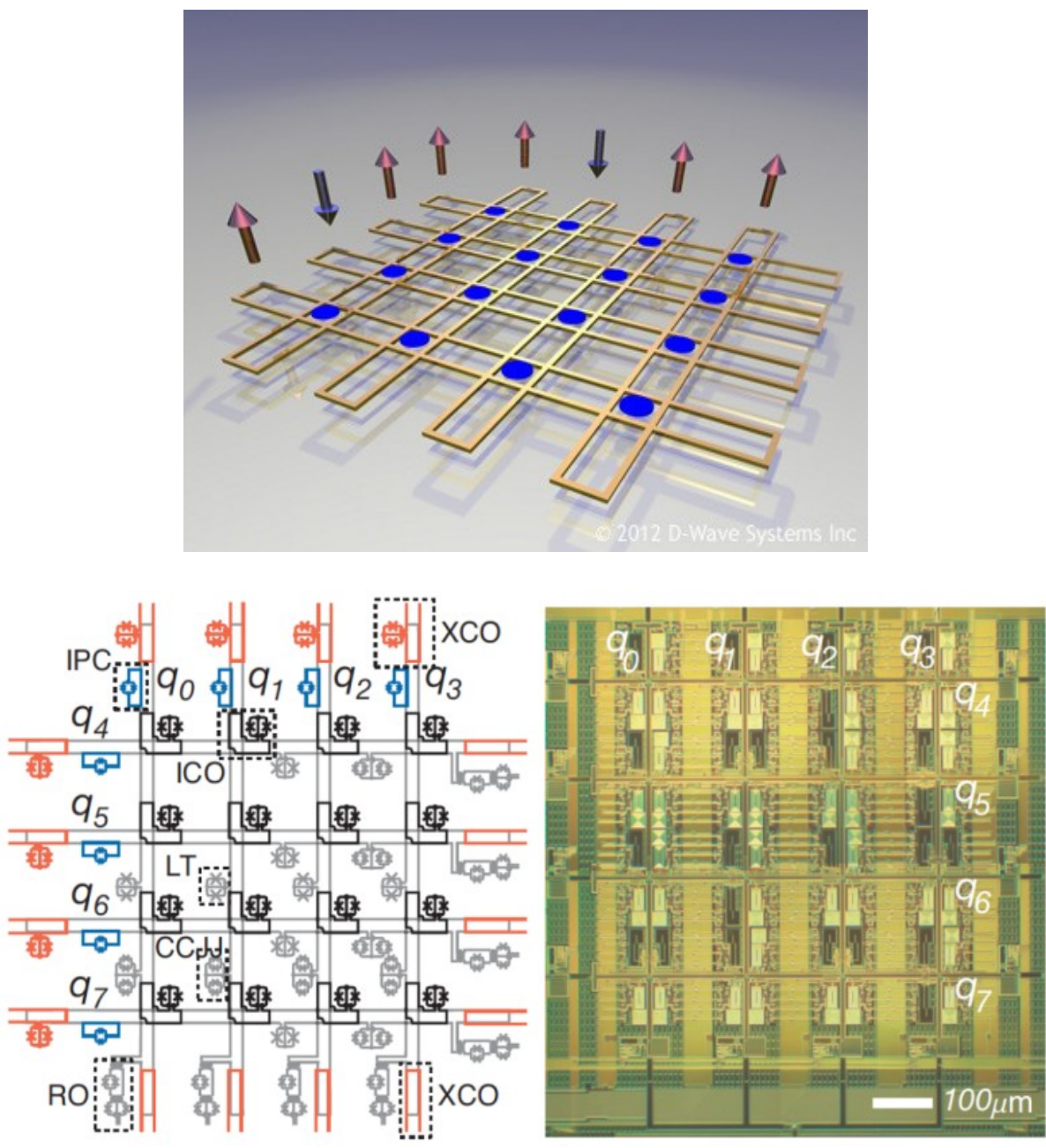

Figure 3: Drawings and photograph of 8 Niobium qubits. Source: D-Wave Systems.

many questions about the systems limitations remain. The consequences of a sparse architecture will be returned to later in the chapter.

While quantum effects are usually associated with extremely small particles, the circuitry in which they are isolated in these superconducting niobium qubits is not particularly small by contemporary chip production standards. The qubits are stamped on silicon wafers, each wafer having many full chips, using foundry techniques pioneered for semiconductor integrated circuits in the 1990s. The 
semiconductor industry has already solved many of the issues for fabricating such circuits, providing a technical and commercial resource legacy that D-Wave has been able to integrate as part of chip production. Developed for other applications, the placement of chip components (e.g. signal lines) and the construction of its subsystems for input/output communication (e.g. demultiplexers, signal routing and addressing) are adopted from superconducting logic circuitry (D-Wave "Quantum Hardware"). While the concept of adiabatic quantum computing was created, in a sense, "from the ground up" (D-Wave "Background") without regard to the formulations/conventions of digital computing theory, the fabrication of such exotic systems has in this way borrowed and adapted many techniques put in place by generations of digital computer production and parameter creation.

Creating qubits out of these superconducting components seems drastically out of scale with earlier and more conventional expectations for qubits. Trapped ions or directed photons are examples of the extremely small materials proposed for quantum bits in academic research (Pavicic 2006; Nielsen, Isaac and Chuang 2011). The relatively large niobium circuits must be put into isolated and controllable quantum states by a series of extreme steps that remove them from the outside world. If this delicate isolation is compromised then the system faces "decoherence" and any attempted computation is undermined (Kalai 2006). 


\subsection{Negotiating Coherence}

Many steps are performed in a quantum computing network that sort the physical world so that some phenomena constitute information and others noise.

Ordinarily, quantum effects are seemingly absent, or at least quite hard to notice, at larger scales than the atomic. Many steps are needed to separate the qubits from everyday existence, so that quantum effects (e.g. superposition and entanglement) can be elicited on the niobium. Otherwise, the niobium grid would appear to act according to classical physics and not be a qubit. A key actant in this process is temperature. The quantum-state and computing performance of the chip is directly linked to its temperature. In order to function, the chip must be below 80 millikelvin (mk), though currently the system operates closer to $15 \mathrm{mk} .^{20}$ This temperature requirement puts the chip " 180 times colder than interstellar space" (D-Wave "2X"). The large imposing enclosure of the D-Wave system and the $10 \mathrm{~kg}$ raygun-esque device at its centre is mostly devoted to this cooling requirement (see Fig 4). Each platform in the structure draws out more energy from the system, in the form of heat. This cooling purifies the processor as a computational and quantum space. If the temperature rises too high, the quality of the information processing is effected.

\footnotetext{
${ }^{20}$ As a comparison to the more familiar celsius scale, the human body is typically $310 \mathrm{k}$ (note: $\mathrm{k}$ not $\mathrm{mk}$ ). 0 Kelvin equates to absolute zero, which is -263.15 centigrade. The lowest natural recorded temperature on earth is $185 \mathrm{k}$. A millikelvin is $1 / 1000$ kelvin.
} 


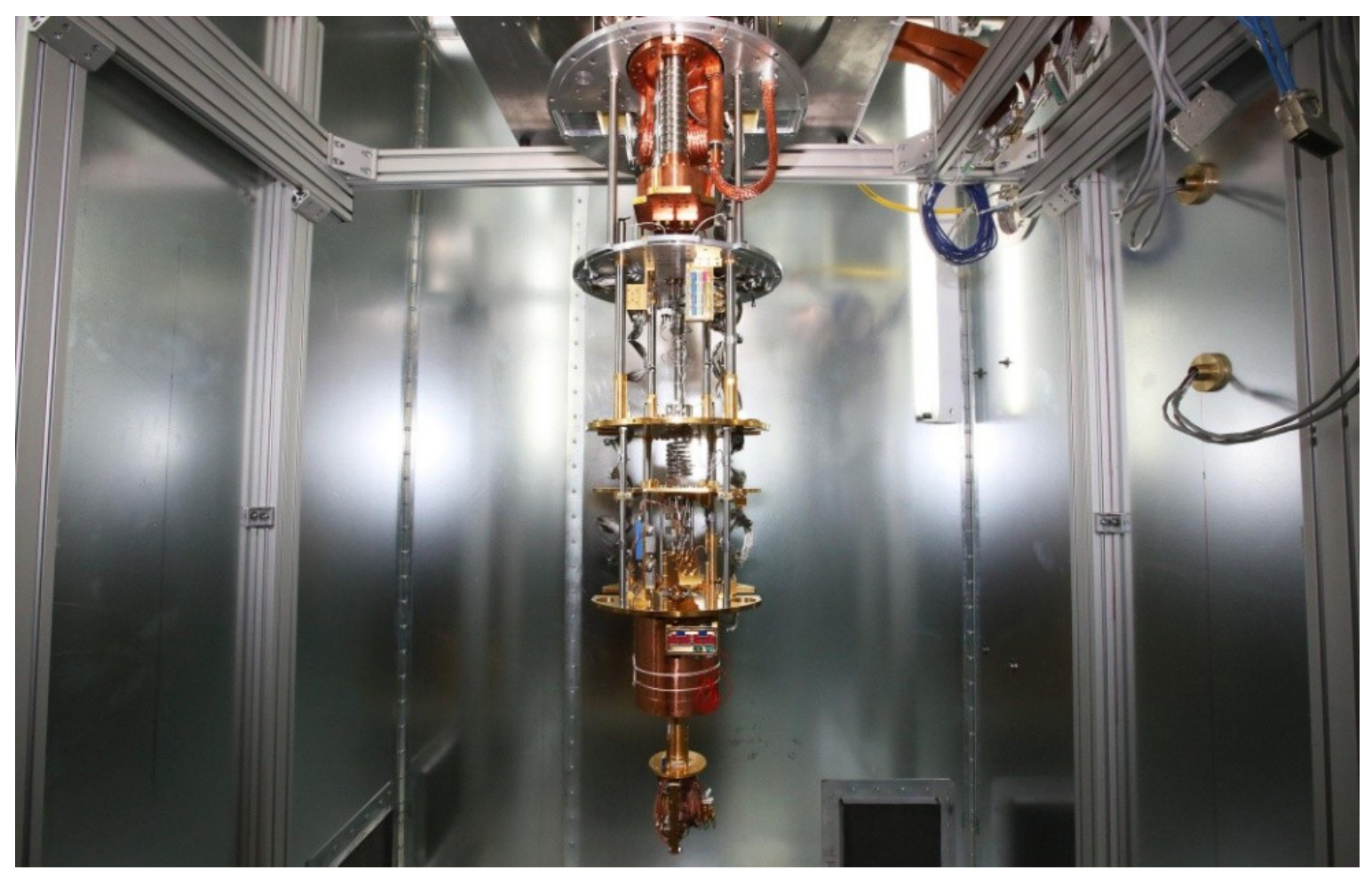

Figure 4: D-Wave interior. Source: D-Wave Systems.

\subsubsection{Temperature}

Temperature has also been an important factor in digital computation devices and their systems. Many techniques have been developed to remove heat from computational spaces both in the design of bits themselves and in creating additional technologies that mediate the temperature of computers and their spaces. Vacuum tubes ran hot, creating system problems that affected their reliability and cost.

Transistors were a significant improvement, scaling for several decades before heat become a common concern in their industrial design and implementation (Hill, Jouppi and Sohi 2000). In the digital-transistor schema, temperature is an 
undesired by-product of the computation, a type of exhaust. Created by the mechanical-energetic interactions of the bits and other material actants, too much heat in the system can raise the voltage and create errors in the bits' computation and information states. Most commonly however, unmanaged heat burns out components such as hard drives and processors, shortening the lifespan of computational systems. To maintain the ever increasingly intensive processing power of digital computing, many cooling systems have been put in place. Personal devices, data centres and supercomputers have taken different approaches to dealing with this temperature-problem. Fans move heat away from chip boards on laptops, and direct AC-produced cold air is circulated through raised floors in data centres. Servers are arranged in rows to produce hot and cold aisles to optimal direct temperature flows. Liquid freon refrigeration, physical heat 'sinks', raised ventilated flooring, naturally cold climates, and full immersion in non-electrical conducting liquids are just a few of the techniques deployed to direct and mitigate temperature in common digital computing systems. Supercomputers and data centres running clusters of processors on single problems (e.g. Amazon's EC2, Google's Cloud Platform) highly refine the temperature-problem as part of their operation procedures, as opposed to the haphazard cooling solutions found in smaller hosting/processing companies. These advanced systems increasingly use water to deal with heat, by piping it through rooms and computer cabinets, in some cases spraying it into the air. Occasionally, particular exotic systems will repurpose this exhaust heat into energy production for heating other components of a building. Much more 
typically, temperature is an ongoing battleground for digital systems. The human managers of computational spaces routinely shut down equipment as heat rises. Heat failsafes that should automatically shut down components as they reach critical temperatures are known to be unreliable in practice for large scale computing (Miller 2012b). In these arrangements, temperature and computation are resources that must constantly be balanced by systems managers, engineers, and of course, accountants. Cooling costs. The prescribed temperature of the most 'advanced' digital computing spaces at companies like Facebook and Google had been on the rise, sacrificing component lifespans for overall saved expenses (Park 2010, Miller 2011, Clancy 2012, Miller 2012a). Temperature becomes mobilized by digital systems as a phenomena that is put into contact with other categories in a computing network. A naturally cool environment, for example, must be weighed against access to transmission infrastructure, hydro rates, tax incentives, etc. Temperature can collapse a digital computer by burning through it, but it in this way it is also collapsed as a variable in the larger digital computing paradigm.

The result of this management and mitigation puts the temperature of digital computing on a spectrum from approximately 15 to 32 Celsius. A relatively comfortable range for a human. The fluctuation of temperature as part of a costperformance metric has had the networks run by Google and Facebook rise to approximately 26 degrees and higher in recent years, to the point data centers sometimes separate the working spaces for human managers from the machine spaces, as the humans are getting too warm (Miller 2011, Zimmerman 2012). 
Temperature might not quite be a bug, but it is neither a feature in digital computing. It is an actant which much be constantly facilitated and a site of struggle for maintenance, budgeting, and design. It is absent from the diagram of the Turing Machine, but a critical aspect of its industrial reality. While heat can slow down computation, heat removal is not depicted as speeding computation up, but rather maintaining it.

The relationship to temperature is completely rethought in the quantum system. At under -273 degrees celsius, .015 degrees above absolute zero, the qubits' temperature is of a different magnitude, and plays just as different a role. In order to reach these temperatures the system uses dry-dilution cryogenic refrigerators stocked with liquid helium. The helium is used in a "closed loop cycle" where it is recycled and recondensed using a "pulse tube cryocooler" (D-Wave " $2 x$ "). In order to rely on this refrigeration technique, which was previously only used in research environments, new coolant techniques were developed in parallel with computer processing rather than as a compensatory afterthought. Qubit production required this refrigeration technology to achieve cooler temperatures and to maintain it over longer periods of time, as well as in the self-contained systems suitable to the remote deployment of commercialized products. ${ }^{21}$ The qubits' materials can be fabricated without this intense temperature control, but the negation and controlled fluctuation of temperature is required to turn the

\footnotetext{
${ }^{21}$ These cooling advances are as patented as the qubits the enable, see: "Systems and Methods for Cryogenic Refrigeration" for an example USPO document. https://docs.google.com/viewer?url=patentimages.storage.googleapis.com/pdfs/US20140137571. pdf
} 
niobium into a qubit and have it process information. The energy program of quantum annealing requires the careful lowering of this kind of energy as the qubits settle into optimal states in their energy-based computation. This is in part what replaces the binary logic program, moving computation from a logical relationship to a material one. Far from exhaust, temperature constitutes the computational relationship and helps induce the production of new information. Simultaneously, outside heat is reconceptualized as noise, interfering directly in the quality of computed results.

\subsubsection{Magnetism}

The other central energy factor to the computation is the role of magnetic fields. Strong magnetic fields have been known to have negative effects on digital transistors since the 1960 s. Their role however is relatively minor ${ }^{22}$ in most digital systems on Earth (less so in space), acting occasionally as a source of interference. As mentioned earlier in the chapter, magnetic fields serve a different role in a quantum system. By assigning bias to the connections between qubits, magnetic fields also become a means of information input and a system "control knob". The magnetic fields in the system that act as an information source and control knob for the qubits are classified and separated apart from magnetic fields not intended to be part of the computation, ordering some fields into being control mechanisms and information, while others become a new form of environmental noise. Qubits are protected from stray magnetic fields by layers

\footnotetext{
${ }^{22}$ External to their role in producing voltage.
} 
of physical shielding. The use of non-standard materials in the input/output, such as tin, are similarly needed to avoid magnetism and be able operate at temperatures below $20 \mathrm{mk}$. A system of 5 superconducting and high-permeability metal shields reduces fields around the processor to 1 nanoTesla (nT), with remaining ambient fields reduced to zero by deploying countering magnetic fields. This is 50,000 times less than Earth's magnetic field (D-Wave "2X"). The resulting vacuum from these isolation strategies has 10 billion times lower pressure than the atmospheric pressure of the room the computer sits inside. The chip is now dead. ${ }^{23}$ A void of heat and magnetism, set as far out from the experience of the outside world as technologically possible. Through the labour of all this isolation, the novel effects of quantum states establish a unique environment, built to house carefully formed relations that process information by their inherent being and environmental forces. The construction of a purified space for quantum computation requires this extreme isolation.

\subsubsection{Power}

As digital computing power scales, its temperature rises and in turn raises the costs and power requirements of computation. This relation has been an influential factor on the industry as it significantly restricts technologies, maintenance schemes, and the geographic placement of computation. The power consumption of the quantum computer scales very differently. The resources required to cool each computer is barely affected by its processing

\footnotetext{
${ }^{23}$ Or at least, anything touching it would be.
} 
power. This is because the work to create the extreme cold environment is the same regardless of the number of qubits in a system. Each processor requires under 25 kilowatts $(\mathrm{kw})$ regardless of the size of chip, while traditional cooling for digital computation with a supercomputer can easily require thousands of times more power for cooling. The advent of server farms and supercomputer centres has created increased critical attention to the material and energy footprint of such systems (see: Niyato, Chaisiri, Sung 2009, Swanson 2011). While I do not address the environmental impact or economic savings per computation of comparing such systems here, it is worth noting a potential break in the link between scaling of energy consumption and high-load computing power.

\subsubsection{Chimera Graphs}

Qubits are hidden from human view. The chips containing them are placed in large black casings, standing 10' cubed. While niobium goes through its annealing process to perform a computation in a quantum state, it cannot be monitored in any way without disturbing the computation process. To pass qubits instructions requires a number of technological media such as digital optical channels and programming languages. The difficulty in knowing how to develop the novel kinds of instructions required to effectively use niobium qubits is reflected in the running joke by its programmers that when a new client got theirs delivered all they really had was a $\$ 15,000,000$ fridge. In order to design algorithms and parse them into instructions, researchers needed a more accessible version of the qubits that reflected the sparse connectivity of the 
niobium qubits' architecture. This representation of the chip was developed by DWave, who translated the chip into a graph diagram. These became known as Chimera Graphs and were circulated as part of tutorials for how to use the machine, and became common tools for programmers to think through concepts of how to match the qubits to variables in the problems they were trying to solve. Chimera Graphs were printed out onto custom whiteboards on several walls of 1QBit's offices for such brainstorming, and were frequently reproduced in company documents. Chimeras consist of a rectangular grid of nodes and connecting lines, where each node represents a qubit and each line the physical connection where the rectangular loops of niobium overlap and can share information (see Fig. 5). Translating a problem's variable into the connection of qubits represented on the Chimera is referred to as "mapping" and "embedding" the problem by researchers. If a problem can be mapped/embedded onto a Chimera then researchers assume the machine can solve it.

The Chimera Graph is not a diagram of the chip; zooming in on the circuit would not resemble this structure. Rather, it restates the possibility of what problems can be solved on the chip by showing the logical relationships between qubits and possibly the reach of their potential entanglement. ${ }^{24}$ This reproduction of the qubits, while representing them abstractly in a useful way, also creates an

\footnotetext{
${ }^{24}$ As explained earlier, qubits share information between qubits to determine their end states (via entanglement). However, entanglement is not necessarily limited to the qubits shown as directly connected on a Chimera. The extent/range of entanglement in the system is a point of contention and ongoing research (Rønnow 2014). How 'logical' these connections are by the standards of philosophy and data science is an intriguing question beyond this chapter, addressing which would require examining the standards by which technological systems are ordered into layers of
} 


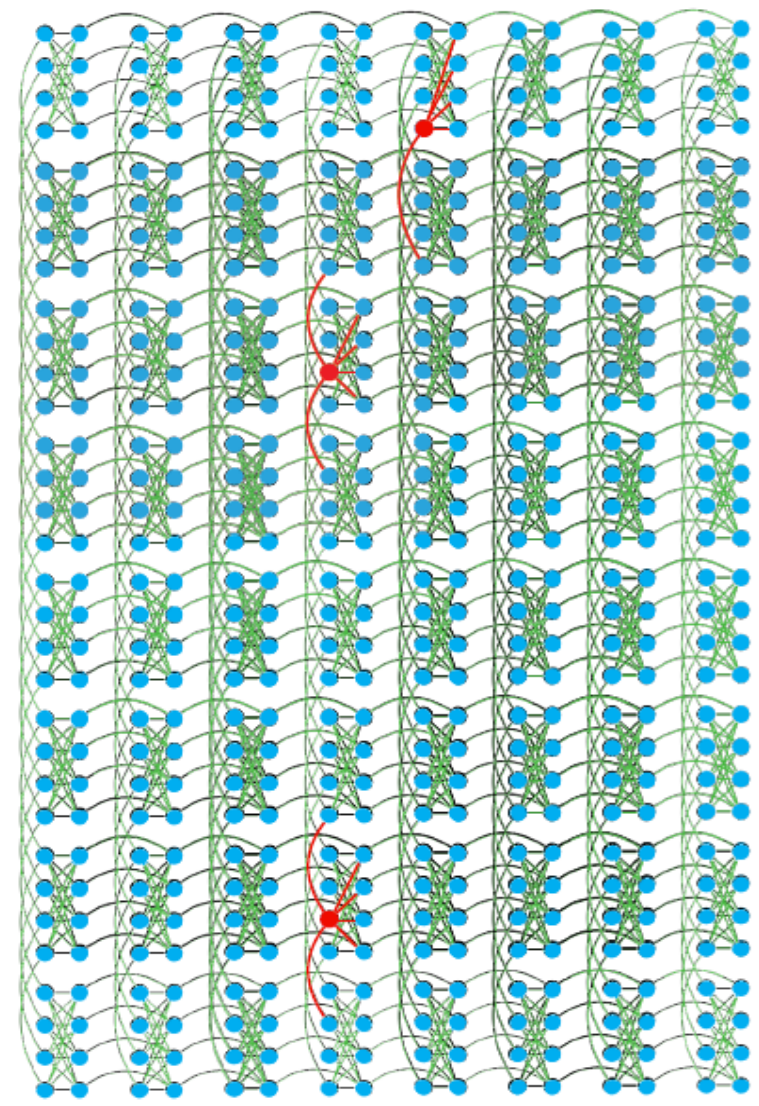

Figure 5: Chimera Graph of 512 qubit chip with 3 faulty qubits. Source: D-Wave Systems.

expectation divorced from the physical machine. The use of this graph as a way to depict potential mappings has led to frequent references by researchers at 1QBit and D-Wave to an "ideal D-Wave". In practice, any individual chip has manufacturing defects that result in broken qubits. These broken qubits cannot be used in the computation and, to varying degrees, break up the possible connections to be made on the Chimera, limiting the chip's usefulness. Some problems may be made to fit on an ideal D-Wave which may never work on any actual D-Wave of the assumed number of qubits. The ideal machine is a useful assumed but inconsistent connector/protocol. 
fiction that provides a standard expectation; it makes it easier to design algorithms that eventually would work on a machine with larger numbers of qubits, and therefore less crippled by random faulty bits. Algorithm design requires assumptions about the technological system that run them. As the quantum system produces information through new processes, new algorithms become possible, and others more difficult to implement. The ideal notion fosters the investigation of a generalized set of embedding techniques that 'place' an algorithm on qubits with a default system of logical/relational assumptions. This is separate from the process of tailoring problems to particular chips, errors and all, which is currently a daily reality for quantum annealing.

\subsubsection{Logical Qubits}

The above sections depict two main identities for the qubit for programmers. First, there is the conceptual qubit. This identity emerges from the mathematical descriptions of " 0 and 1", the algorithms enabled by a non-binary bit, and the theory of quantum computation. Secondly, there is the physical, material qubit. A piece of niobium with specific properties and requirements that is made to act as a qubit through a process of isolation and connection. It is correct to say that a qubit is a junction of niobium supercooled rods, connected into a network of such junctions as described by the Chimera Graph. It is also not quite that reliable a definition. A third qubit identity emerges from the practice of merging the theoretical and material qubit. This version of the qubit reflects a roaming functionality that can be reassigned to smaller or larger physical systems 
depending on the needed agencies/connectivity of the problem being computed. Researchers at $1 \mathrm{QBit}$ frequently try to map problems onto the chimera graph that include variables that have many needs. These needs may include mathematical constraints or other relationships between variables in the problem that the sparse connectivity of the niobium qubits cannot faithfully represent. In these moments, researchers may either need to consider the problem as unsolvable with this machine, or, mobilize the qubit as a logical identity that can be reconstructed in another way. This involves designating a sub-network of niobium qubits to act as single qubit for one variable, benefitting from a greater level of connectivity and therefore applicability to more problems. This grouping technique has a pragmatic limit, as it quickly uses up the finite number of niobium qubits on a chip. Further, creating long chains of these mega-qubits has the potential to degrade the computation as it strains their connectivity.

The amount of qubits on each chip has been growing at a fast pace, in many years doubling. While each chip only had 128 qubits in 2010, this reached 1024 qubits per chip in 2015. Complex problems require many variables and so many more qubits to represent them. However, as the available number grows it allows this specialized quantum annealing computer to become, to a degree, more general-use and imitate other architectures as the number of niobium qubits increases. This disconnecting of the physical qubits from a 1:1 relationship with the abstract variable of a problem makes their identity increasingly fluid and contextual. A qubit is not necessarily either the clear-cut unit where one physical object in the computer architecture cleanly represents one mathematical term, or 
the theoretical qubit supposed in a quantum algorithm. This differs greatly from the way a digital bit represents a transistor. What acts as a qubit is negotiated and reassembled by the algorithm/function and the researcher. They are constructed and "made to appear" (Latour 2010) by a series of alterable relations rather than purely a set system of physical engineering. The important aspect about a qubit are its properties and assigned relationship to other qubits, not a stable placement in the machine. The chimera graph can be redrawn to be faithful to the idea of a qubit in an arbitrary algorithm written by a researcher at 1QBit, instead of the physical architecture manufactured and described by $\mathrm{D}$ Wave Systems. This redrawing often begins literally, circling grouping of qubits into single nodes with marker on chimera-whiteboards. The diagram that emerges appears as a large game of overly complicated Tetris. The embedding process can be so complicated that it itself qualifies as an NP-hard problem, benefitting from the help of the quantum computer, to help process its own embeddings.

\subsubsection{Benchmarking and Comparison}

The arrival of niobium based qubits into the computing world came with a great deal of controversy. Disagreements about their traits, especially how they will behave in larger chips with thousands of connected qubits, continues to be a subject of debate. Benchmarking has often been looked to as a way to describe the qubits as effective, reliable, and ultimately as real, both by proponents and critics of the technology. Benchmarking provides two functions for the qubits. First, it potentially establishes that they actually are qubits, showing speedups or 
results that indicate entanglement and superposition are taking place in the computation. Given the difficulty in observing any quantum computation in-action, and the suggestion that non-quantum properties might account for the niobium's computational abilities, this has been a significant role. Second, benchmarking provides a way to begin to compare quantum bits and digital bits as computational competitors, not just alternative models. From 2012 to 2014, DWave began giving researchers access to their hardware to produce objective benchmarking that would establish the machine's speed and quantum nature. Three separate studies initially were produced. Though some of these papers have been published in well known science journals, such as Nature, the majority of academic articles from both 'sides' have appeared on the e-print service arXiv.org, run by Cornell University. In 2012, a team led by Sergio Boxio ${ }^{25}$ showed that the behaviour of a 128-qubit chip matched the behaviour expected of a quantum annealer, and was widely cited as evidence the D-Wave was quantum. In 2013, McGeoch ${ }^{26}$ and Wang produced benchmarkings for D-Wave, comparing it favourably to a classical optimizer. The frequently cited takeaway from her study was that, "The D-Wave quantum computer processor is 3600 times faster than classical computers at some tasks" (Jones 2013). This figure received widespread circulation with little context provided to the conditions of the speedup. In 2014, Matthias Troyer published results of his findings for

\footnotetext{
${ }^{25}$ http://arxiv.org/abs/1304.4595

${ }^{26} \mathrm{McGeoch}$ is a Professor at Amherst College, but was also hired as a consultant for this work by D-Wave.
} 
benchmarking a narrow category of problems on the D-Wave Two. ${ }^{27}$ His results indicated the machine was working based on quantum mechanics, but was skeptical whether this model would offer a significant speedup for computation.

These studies and others have been challenged by many academics on various grounds, without definitive conclusion, and with papers still appearing. Some notable instances include when Boxio's study was refuted by a team of computer scientists, including Umesh Vazirani (Shin et al. 2014), who argued a classical non-quantum model could account for the D-Wave processor's behaviour (which in turn has been argued against). ${ }^{28}$ Also, researchers who considered McGeoch and Wang's comparison to be unfair created a custom-written software that outperformed the D-Wave at optimizing the specific problem with which it achieved the 3600 figure (generating a wider discussion of how to fairly benchmark such hardware) ${ }^{29}$ The ongoing article back and forth has provided DWave supporters with solid ground to make their case that the computer is quantum in some sense (Vinci et al. 2014), but with many skeptics still on the fence about what is happening inside the giant black box that surrounds each $D$ Wave processor and what it can be made to do. ${ }^{30}$ These debates have largely stayed inside the academic community, but become part of a reputational issue that the qubits' spokespersons must in some way address to the corporations

\footnotetext{
${ }^{27}$ The "D-Wave Two" refers to the 512 qubit system. It is also referred to by the name of its processor chip, Vesuvius.

${ }^{28}$ http://arxiv.org/abs/1403.4228

${ }^{29}$ This debate too was largely had via Scott Aaronson's blog, with Cathy McGeoch participating.

${ }^{30}$ A significant factor still up for debate is how broadly entanglement can occur throughout the chip. If only qubits very close to one another can be entangled, this would be a significant limit.
} 
and organizations they aim to enrol. The existence of this debate also results from a deep seeded difficulty in comparing digital and quantum computation.

\subsubsection{Time}

The best method for assessing the comparative speed of a quantum computer with established digital methods is still under debate, in part because the number of qubits is still too low to enable a full comparison on problems that pose great difficulty to optimized digital machines (Rosenberg et al. 2016). Fig 6 shows a graph from a 1QBit research paper, depicting a comparative benchmarking exercise. The red line labelled "hybrid solver" depicts the performance of the quantum computer (see the following chapter for further explanation of the "hybrid" approach). The X axis shows the number of variables in the type of problem being benchmarked, with the $\mathrm{Y}$ axis showing the time it took to find the answer. The other coloured lines on the graph represent a range of configurations of Gurobi. Gurobi is a well known industry-leading digital solver for optimization problems. The graph does not show a clear winner, but, more interestingly, displays the innate differences in how computations with qubits and bits relate to time. Attempting to show the benefits of the quantum computer with this graph is seemingly impossible. The red quantum line ends at 23 variables, before its digital competitors begin to show slower solution times. The abrupt line ending of the red line's trajectory is because the number of qubits on the chip only allowed for this many variables to be represented, which is just below the threshold at which exhaustive digital computing techniques begin to experience 
dramatic slow-downs. The quantum computer even shows noticeably slower processing times at 17 variables and below. This reflects the fixed annealing time the quantum computations require regardless of how many variables are being run on any number of qubits. Since the annealing process is a fixed analog event, this means the processing time does not increase with increased computational complexity from increased numbers of qubits/variables. A digital computer by comparison, has essentially an unknown processing time until it has finished processing. Digital processing times are found by experiment, whereas the quantum system assumes time as a static variable. If it can be embedded, the solution time for a problem is known, as it should never change. A generalized processing time is an unexpected feature of a specialized processor, that is otherwise an obtuse blackbox, difficult to see inside. The scaling of digital computers was motivated primarily by increasing their speed to enable them to take on more and often more complex computational tasks. ${ }^{31}$ Scaling quantum computers is instead linked to increasing the complexity of the problem they can be made to solve by increasing chip size. Moore's Law described an increasing density on a stable chip size, whereas the scaling of quantum annealing at this point keeps the qubit size stable and increases the chip size in response. ${ }^{3233}$ Instead of solution time as a core metric, this shifts the focus to the size of solution space; increasing the volume of the landscape the qubits can map.

\footnotetext{
${ }^{31}$ Though increasing mobility and reducing cost were also important pressures for transistor miniaturization.

32 The doubling in size has at times been called "Rose's Law" by D-Wave, after its CTO and Cofounder Geordie Rose.

${ }^{33}$ Other quantum computing models do propose much smaller qubits, continuing the miniaturization trend of digital computers, but these have yet to be constructed.
} 


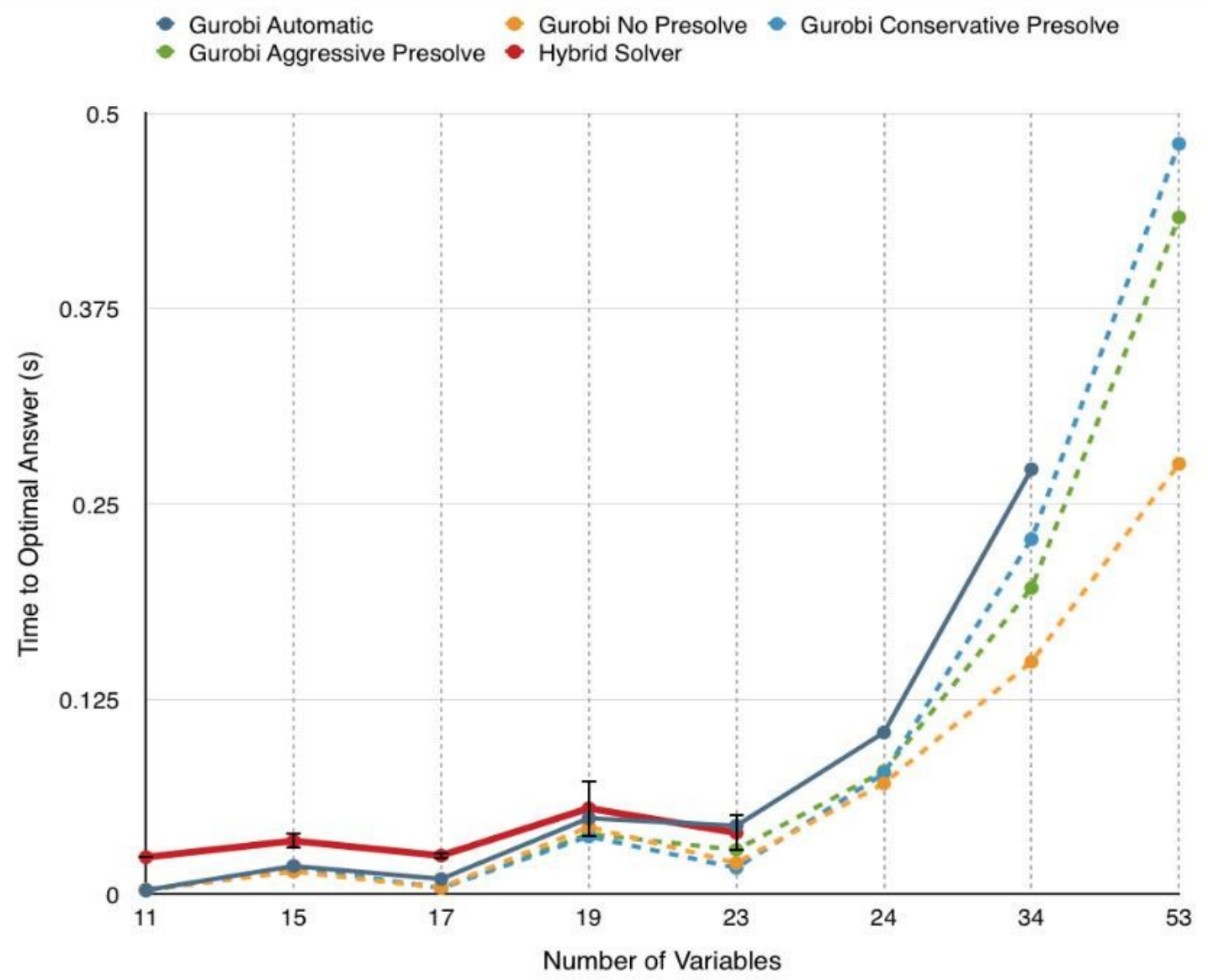

Figure 6: 1QBit benchmarking results. Source: Zaribafiyan and Oberoi 2015.

\subsection{Conclusion}

Qubits signal a major departure from the assumptions of digital computing, and the expectations, practices, and abilities that have grown around the digital. The qubit is a new building block for computation that combines concepts from digital and analog systems to establish a new logic of computation and set of actors at play in computation. Many materials and techniques are brought forth from the digital, but others are radically reshaped, including the role of basic physical forces such as temperature and magnetism. The mathematical/operational logic of the digital cannot account for the new computational methods described here, 
and so we are invited to broaden our understanding of what counts as computations and computers. The process of constructing, isolating, connecting, and circulating qubits, and bringing them into the larger world of machines and human programmers, shows the breadth of environmental and technological ordering required in a computational system. Quantum computing generates a new series of meanings, roles, and identities for nonhuman and human actors in computational systems; following the qubits' role in this system shows many of the dimensions in which the assumptions and approaches of the digital must be abandoned for new, initially strange, computational networks.

This chapter's gathering of the plurality of distinctions between the digital and the quantum demonstrate how the technical materiality of a piece of hardware is assembled out of many heterogeneous actors and relationships. The qubit holds together as an actor due to the work done to construct it, reordering and redefining phenomena both inside and outside of computers. These many distinctions reveal the broader distribution of agencies and identities that make up qubits and enable a quantum computing network. Qubits are important actors in this network, and technical accounts of their engineering give them significant attention. The gathering of distinctions required to construct AQC models of computation, however, calls attention to the ways their resulting agencies can be negotiated with to be put into particular computing practices. The following chapter will examine this negotiation in the context of software development and the problem-solving processes deployed to negotiate with the constructed agency of qubits. 


\section{Chapter: The Portfolio and the Processor: Negotiating Problematics to Enable Quantum-Hybrid Software}

Qubits are difficult coworkers. Many problems were navigated in their engineering, and the concessions required for them to emerge as functional components creates significant challenges for their programming. To program them means dealing with a problem of problems: they compute a particular relationship, but we have many kinds of problems we would like them to solve out in the broader world of humans and industries. To do that requires finding a way to make the world computable through the affordances of its new qubit occupants. This goes beyond the routine observation of the digitization of the social and material world (see: Dellarocas 2003, Davidson 2005, Lahlou 2010, Katz and Koutroumpis 2012, Newell 2012), and poses questions around how computational frameworks come into being, what problems and solutions they recognize as computable, and how these evolve out of the daily concerns and practices of specific actors.

In this chapter I examine how a hybrid model for quantum computation was developed. It demonstrates how software programming in quantum computing is developing by interacting with the commercial and social conventions of institutions, as well as the limitation of hardware resources. I argue here that software development becomes a negotiation point at which a problematic is formulated that then acts to shape and grow the network of quantum computing. 
It shows how a socio-technical computing network works to reshape the world into a favoured type of computable problem. The domain of software is in this case indeterminate, coming into being through the agencies of distinctly nonsoftware actors before familiar programming conventions are established. Centering the problematic as a key figure in analysis bridges ANTs notion of following actors with its description of how actors' patterns of work are established as visions and then performed routines, in what it terms "programs of action" (Callon 1986). Thinking in terms of problematics ties the accomplishment of work to broader desires, goals, and worldviews that motivate and create a sense of urgency to establishing networks.

Dealing with specific problems has led to many key developments in computation, and generated new methodologies and systems for information processing. The process of problem-solving the limitations and uncertainties of technical systems has been considered by some scholars for its role in expanding and evolving infrastructure and technology (Hughes 1985, Bowker 1994, Edwards 2010). How the process of grappling with low-level or specific problems shapes the growth of computational systems, is occasionally mentioned in accounts of computer development, but usually as a piece of background information rather than considering problems and the process of forming problematics that encapsulate and solve them as a driving force or actor. For example, descriptions of how high-level computer languages evolved from libraries of subroutines assembled to solve specific problems (Ceruzzi 2003, 108); the development of time-sharing in conjunction with the information 
processing needs of the 1960s space-race (117); or the conventions of TCP/IP arising from military directives for problem-solving communication lines and increasing "robustness" for what became ARPANET (Ceruzzi 2012, 122), tends to take a backseat to lineages of machines, people, or theories (Edwards 1988). Hughes (1985) in particular suggests greater attention to the role of problemsolving in technological development, that highlights the role of "sweet problems" and tracing how initial technical developments become "solutions looking for problems", that then expand technological systems in their hunt. Here I take up this perspective and aim to follow the problem, as it becomes a central actor in the quantum computing network. This expands what we can consider as software related activities, and show how problematics are a neglected aspect of socio-technical networks, one that is neither cleanly material-technical or humansocial, that significantly shapes computation.

\subsection{Separating Software}

As suggested in previous chapters, the majority of research and media coverage of quantum computing has been on the development and 'clocking' of hardware configurations. There has been less said about what a quantum program or application may resemble. A notable exception is Shor's Algorithm, which has developed an important legacy in quantum computing. Shor (1994) developed a solution to the integer factoring problem based on the theorized parallelism of quantum bits. Factoring describes taking a number and finding two numbers that multiply to produce it. Shor's Algorithm does this quickly when using a quantum 
computer,${ }^{34}$ requiring only a short number of steps even for very large numbers. Shor's Algorithm quickly became central to the notion of a quantum computer, in part because of its illustration of the difference in magnitude of computing power between a classical and quantum computer, as well as its implications for common computing practices. The ability to quickly factor such large numbers is in effect a way to undermine the 2-key encryption system that is used to provide security in digital messages. Any current encryption system could be quickly cracked. ${ }^{35}$ This exercise in cryptography became a de facto test for a quantum computer. For example, IBM has demonstrated a rudimentary 7-qubit computer by factoring the number 15 into 3 and 5 as validation (Vandersypen et al. 2001). ${ }^{36}$ Shor's algorithm assumes a "gate" model quantum computer. Logic gates are a foundational feature of digital computers, acting as switches that perform boolean functions on bits. A quantum gate manipulates the state of a qubit in a similar way to the logic gate in digital computer. This version of a quantum computer, made popular by descriptions such as Feynman's and Shor's, is for all its differences a clear extension of the digital computer's model and approach to problem-solving. It merely adds more functions that gates can account for with superposition.

\footnotetext{
34 In Polynomial time.

${ }^{35}$ For this reason, popular media coverage of quantum computing frequently involves a discussion of cryptography and speculated on the involvement of the NSA.

${ }^{36}$ It's worth noting that IBM's prototype is not agreed upon to be an entangled quantum computer.
} 
However, virtually nothing has been written on the actual process of quantum software development done to date. Establishing this new sector of the computing industry requires setting apart a set of specializations from now apparently separate segments of technology that in practice remain intermingled. This dynamic has been a part of the growth of the computing industry since its mid-1900s boom. As a term, "software" dates to the late 1950s. However it was in the late 1960s that it became operationally disentangled from hardware into its own business sector, a set of practices, and a technological division; this was thanks in no small part to the computing industry giant of the time, IBM, responding to legal pressures from antitrust decisions to segment its business (Katz and Shapiro 1999, Fuller 2006). This business/legal separation has been reinforced by the development of software engineering and programming languages that do not directly interact with hardware. Software is often visualized by these practices as a secondary layer that sits "on top" of the hardware layer (see Fig 1). The legacy of digital computing has resulted in software typically being further divided into two types on this layer, with that layer sometimes further divided into sub-components as programming practices have become more sophisticated and often removed from engineering. The first of the most established layers is systems software, which enable the computer to perform its basic functions. The second, and generally more familiar to the average user, is applications software ${ }^{37}$ Applications software consists of what users experience as the content of computation. These are the particular operations and data that

\footnotetext{
${ }^{37}$ The softest of the soft.
} 
general-use computers are specifically imitating for any given operation. In most cases, applications software is dependent on the existence of particular systems software, which in turn requires certain hardware conditions. This is quite familiar to anyone who has tried to install a .DMG file on a Windows PC. However, many examples of applications software do exist on different systems software and hardware configurations. It is not unusual for a program to be written for a Windows PC, a Mac, as well as having smartphone variations. In each case, the software has been reconfigured for a different technical configuration. Often it is not a challenging technical task ${ }^{38}$ to adapt an application for these different systems, with the main barrier usually being a legal one. This is because a piece of software is built around an algorithm (or set of algorithms) that at their base assume some kind of Turing Machine. ${ }^{39}$ Software is in this way as deeply tied to its formulative algorithms, distinct from their various encoding in computer languages, as it is a hardware configuration. Even though separate from programming language, the algorithm itself is not a completely abstract entity. At its lowest level it assumes a type of architecture executing it, and is in this way grounded in some sort of physical system. Until recently, this would exclusively be assumed to be a digital architecture. ${ }^{40}$

\footnotetext{
${ }^{38}$ An exception being when smartphones with generally fewer resources try to run larger programs, or as is often the case, parts of them.

${ }^{39}$ As well as data with which to operate.

${ }^{40}$ There have been many historical non-digital computers, some of which competed with modern digital computers into the mid 19th century. These either were ancient calculating devices or quickly became dead-ends as the digital rapidly overtook computation as the paradigm of computation (in Fuller 2008).
} 
Application Software

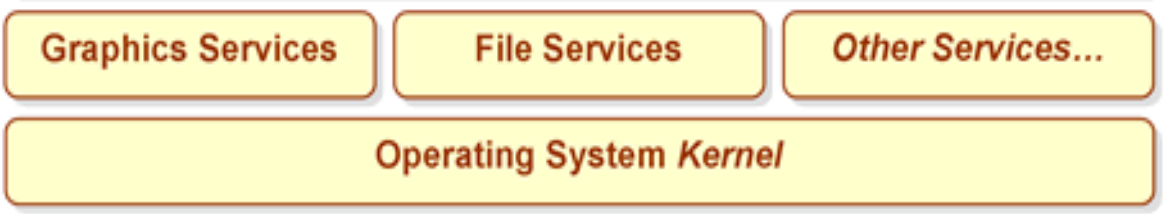

Computer Hardware
User Application

Operating System Services

Hardware

Figure 7: Digital Computer Layer Visualization. Source: MIT.edu

Quantum computers appear to challenge the image of software split between system and application, though as we shall see it has not disappeared in practice entirely. By definition, they also remove the foundational assumption of an available Turing Machine. Current commercial quantum computers are an analog annealing process. They do not 'run' an algorithm in a way similar to a digital computer. Algorithms in a digital paradigm are following a predetermined set of steps that efficiently solve a problem. Software describes these steps in code and tells the computer how to perform them step by step. However, a quantum annealer does not run any kind of code. There is not a central CPU enacting one software command, one step of the algorithm at a time. Rather, it is a physical process that can be given certain conditions and begun, and whose end result can be observed. Rather than a digital clock programmed to count an hour's worth of seconds, it is akin to turning over an hourglass and relying on sand and gravity to get it right. ${ }^{41}$ With this approach what can be said to be programmable is not the operations of the system, but its initial starting conditions. This moves the act of programming from the tight command and control of the small steps of

\footnotetext{
${ }^{41}$ A difference being in this case the hourglass is far more accurate than the clock.
} 
binary processing on Turing's ticker tape, to the crafting of a computational environment that can be controlled in new ways. In practice, this would mean the ability to set the strength of various magnetic fields around the systems of qubits described in the previous chapter. These settings indicate the desired problem, and through the annealing process, result in a new energy landscape. Such 'setting up' is not clearly system or application software, but an overlap of the two designations that requires writing algorithms that address both of these previously divided software types/layers together. How then does one create software for such a system?

\subsection{Gathering Problems and Discussants}

In order to explore this dynamic, this chapter will examine a series of roundtable conversations, typical of many observed during my fieldwork at a software development company for quantum computers: $1 \mathrm{QB}$ Information Technologies (1QBit). The roundtables described here occurred over the course of three months, with the majority being held in the first half of that period, and then becoming increasingly spaced out as the group interactions reflect. Later in the development of the company and its projects these roundtables somewhat changed in character and came to resemble more typical office meetings, with more management involvement and formalized structures. However, at this point, they were populated by the majority of the researchers at the barely-formed company, and were conducted in a format closer to a graduate seminar than a corporate meeting. A small group of individuals with advanced expertise and different institutional affiliations gave presentations and led conversation, with the 
majority of the content produced by discussion between the participants in a mostly informal manner. The majority of participants were in fact graduate students, brought into the company through industry-internships and postdoctoral appointments. This greatly shaped the tone of discussion, leading to a mix of presentation, Q\&A, and often lively group arguments, with struggles for control over the scarce whiteboard pens used to flesh out many of the participants' points. In many cases having possession of the whiteboard pen was one of the few things that gave discussion order, providing some control to a speaker when the conversations become increasingly free-form and among multiple participants. Different participants temporarily led some of these sessions to give prepared mini-talks on areas of expertise not shared by the full group. However, the interdisciplinary and uncharted nature of quantum computing meant that no person or field held a consistent authority. In the course of these meetings the conversation turned to many different ideas and questions, but was given initial starting points and directed to specific research questions that were prioritized by the management of the company. Occasionally, management would sit in on these meetings and get updates on the direction of the group's activities, but at this stage of research the graduate students were left to read and argue as they had learned to in academia - if with some pressure to do so at a quicker pace than most courses. 1QBit assembled the team to see if anything productive could be done with their negotiated access to D-Wave hardware, especially to develop practical software that could be used to increase the value of the company. They 
also needed to see whether the skillsets they had assembled would be able to generate ideas about how to work with the machine.

These conversations started near the beginning of $1 \mathrm{QBit}$ s operations in early May 2013, with the group first sitting down in a small office conference room after only a week of being introduced to each other. 1QBit grew rapidly, switching to larger offices once a year for three years. This first office resembled many early start-ups in the area. It was quite worn down, had a pigeon problem that required the windows being closed after hours, and the only conference room was just large enough to hold the 6 researchers and 1-2 observers. This added to the initial casualness of the atmosphere, and made it resemble the space of an academic tutorial or seminar even more. Also, similar to a graduate seminar, everyone in the discussion had previously been provided with a set of readings by the company. These were mostly articles from academic journals that introduced the basic theory behind quantum annealers and their use of Ising spins to create qubits, as well as early tutorial documents by D-Wave Systems on how the machine was being programmed. Many important papers that led the discussion were later found and added to the group reading list by the participants as they went.

The quick growth of a literature for the group to center discussion around occurred as the participants began realizing that areas in their own expertise, with no well-established connection to quantum computing, were relevant to $D$ - 
Wave's machine. For example, a little known MA thesis on graph theory that explored how the identification of parts of graphs known as quasi-cliques might be used to model and predict market behaviour became a stable reference point once brought in by an engineering student (Jallo and Budai 2010). This thesis had no previous connection to quantum computing, but became an early example of mining optimization and graph theory for techniques that may be friendly to the new processor's architecture. Bringing in papers such as this became a way for the group to develop a 'contact literature'. Turner (2006), described the development of "contact languages" in interdisciplinary technological work that help align the many independent or partially shared vocabularies between fields of research. For example, in one of these early conversations physicists and engineers had to sort out what they were describing as "entropy" in the system. ${ }^{42}$ They came to realize after one lengthy exchange that they had been using the word, which might appear as a somewhat neutral scientific term, in meaningfully different ways. Such debates as well as the creation of new terms as needed helped the group develop the contact language needed for interdisciplinary work. Establishing a group reading list that became maintained on a company server, along with the actual discussions, became an important resource for building and maintaining such a shared language. As an assembled literature it also provided stable reference points in conversation, provided a way to introduce new topics, and eventually created an agreed canon

\footnotetext{
${ }^{42}$ Hayles (1990) describes the complexity of this particular concept in science, suggesting why it may be a likely term to cause confusion depending on its usage. However, this pattern of needing to explore and establish the meaning of scientific terms was not limited to only such likely examples.
} 
of reading within the company as new researchers later joined the team.

Eventually this even morphed into an on-boarding reading packet for new hires, but at this point it was a pile of papers ${ }^{43}$ used to argue about what to do with a strange and seemingly useless processor. Even though D-Wave Systems had produced several generations of hardware, quantum processor technology was still at an extremely early stage. There was very little established literature, few practices around the technology, and no direction on how to search out its relevant problems. This provided the group with the ability to grow the literature into areas they were interested in, but left many questions around what kind of techniques and problems they should be looking for. Very few abstract problems had been solved using the system. There were no non-generic, or "applications" for real-world problems being solved with it that could have been a starting point for the discussions. The extent to which applications could be run on the computer was highly debated within both companies, and though areas of interest had been identified since early in D-Wave's inception and repeatedly promoted as relevant to its technology, ${ }^{44}$ few research directions for software had been significantly explored as hardware engineering challenges remained forefront. Before these roundtables, the 1QBit group had also had some in person tutorials and Q\&A sessions with D-Wave engineers. As the roundtables went on they would periodically meet with D-Wave staff again. In each of these

\footnotetext{
${ }^{43}$ Or more frequently PDFs, though a surprising number of researchers seemed to prefer paper. ${ }^{44}$ A prime example of this was D-Wave's August 2013 press conference announcing the release of its 512 Qubit chip. Speeches to the press showed PowerPoint with icons representing air traffic and similar scheduling problems, security problems, stock market optimizations, and other realworld challenges. At the time no such programs were thought to be possible to run on D-Wave by D-Wave's own engineers.
} 
interactions, D-Wave was openly skeptical whether the architecture of the current processor would be able to handle enough variables to tackle a compelling reallife problem. This was seen as perhaps overly ambitious or premature given the many internal problems being worked out in the machine. Many aspects of its hardware, systems, and programmability were in flux at the most basic levels (e.g. chip structure, system stability, documentation), and in many cases remain so today. With this context of initially scattered resources and mixed corporate encouragement and skepticism, the team could begin to discuss how one set of problems could be connected to another.

\subsection{Selecting Applications for Expansion}

The difficulty in creating real-world applications for the D-Wave system is due to the annealing technique at the heart of its computational abilities. Whereas digital computers are made to solve any potential problem, or rather as Feynman noted a simulation of one, the type of problem this approach to quantum computation ${ }^{45}$ can solve is based on a category of NP-Hard optimization problems. While these optimization problems have many everyday applications, programming them into the computer is not always possible, or is hard to do for specific instances because they contain too many variables to be mapped onto the current generation of processor in the embedding process described in the previous chapter. This complicates the dilemma of finding a problem for that machine that

\footnotetext{
${ }^{45}$ There are many competing approaches to quantum computers. These involve very different architectures (i.e. designs) and media (i.e. materials) that result in quite different computational properties.
} 
must then have both a problem structure, the relationship between its variables, and a specific instance of that problem, which dictates the number of variables, that could be computed. The search for practical real-life applications that the computer can solve is deeply coupled to its basic research and development. As competing methodologies attempt to dominate what is seen as the computational 'holy grail' of a practical quantum paradigm, proving the validity of the approach has been deeply associated in the company with showing it could solve impressive real-life problems. Many early critics of the processor were both skeptical of how mechanically it was performing computation (i.e. was it really quantum), and this was highly tied to showing not just basic computational abilities but advantageous computational results (i.e. quantum-based speed improvements over classical computation). This meant that the researchers working on it had to show this kind of computer could be programmed to do the kind of work that made it worth developing.

Below I examine how translation work is accomplished between a mingling of different actors (e.g. companies, academic fields, individuals) in the process of creating a software method for this approach to computation. Following these translations reveals the methods (and motivations) that gather together into the context of a matter of concern (Latour 2005, 2010). It shows how the technological object comes out of a connecting practice with often unintended consequences, and developing relations is prioritized over consistent planning. Software development in quantum computation arises in this example as a space for the interactions of institutions, researchers, and models. Once these contexts 
have gathered, software emerges as a method for both allocating computational resources and bringing new entities into the quantum computing actor-network.

\subsection{Roundtables}

Around the conference table the 1 QBit team having this discussion is comprised of a financial services expert and a group of graduate students: two mathematicians, a physicist, and an engineer. On the table, are the above series of understandings and expectations of what quantum actors can and cannot do that need to be reconciled for its software development to progress. These six researchers, with myself lurking in the background and sometimes the CEO in the corner, have been brought together by $1 \mathrm{QBit}$ to bridge descriptions of two objects: an option trading portfolio and a 512-qubit adiabatic quantum processor. When 1QBit initially formulated its business model it was around the assumption that these two objects could be aligned in a meaningful way, producing a new computation option for quantitative finance. The majority of the company's pitches to investors and partners depicted the optimization of problems such as the trading portfolio to be innately suited for the computational powers of the processor. The fit of these two objects formed much of the basis for 1QBit's early promotional and descriptive documents, as well as the proposals used to acquire government funding and recruit employees and academic researchers. This connection also fits into the historical interests and research trends of the financial sector. Optimization problems have a long and broad relevance to challenges faced in the financial sector (Markowitz 1952, Black and Scholes 1973, Hull and White 1987, Jackel 2002). The complexity of problems in aspects 
of finance, such as trading and pricing options, has created an ongoing exploration for alternative computing and mathematical techniques to deal with these problems (Meyer 2009, Chang et al. 2009), and to have the mathematical models proposed to describe them adjusted to better reflect the many real-world constraints of actual finance phenomena (Cramas and Schyns 2003). Even slight improvements on such problems can be beneficial. As 1QBit's CEO describes, "for route optimization, if you were trying to win a race then it doesn't matter if you are 1 second ahead or a million seconds ahead, if it's a race you win." The expectations for quantum computers in this area are much higher for some, as can be seen in a blog post by Marcos Lopez de Prado, Senior Managing Director at Guggenheim Partners and 1QBit board member: "once quantum computers are deployed in production, it will be impossible to catch up. Alpha will dry up overnight, and most traditional hedge funds will go bust, as the old inefficiencies disappear. In particular, many high-frequency traders may starve, as large asset managers will produce robust portfolios will little noise-trading" (May 11, 2016).

However, at this point, none of the six have actually seen a D-Wave device, tried to program one from afar, or in most cases had experiences dealing with real-life optimization problems. The one non-student in the mix, the finance expert, does have experience with many of the kinds of finance problems the company would like to solve. The readings the group has been provided before meeting dealt more with the machine's physics than with finance, and there is a desire by the finance expert and management to quickly move beyond conversations covering engineering issues and center discussion instead on translating the finance 
world, particularly trading problems and the kinds of solutions currently employed to solve them. The potentially high monetary value of improving on the solution to such finance problems provided a clear motivator and justification for upfront research and development costs to 1 QBit. As the research team began its discussions the company was also trying to raise awareness, and eventually investment, to support their efforts. As the company entered discussions to take part in a highly prestigious quantitative finance conference held annually by JP Morgan ${ }^{46}$ there became a company-wide goal to connect these actors in a meaningful way at an accelerated pace. Despite this sense of urgency, the discussion can not quite skip to this area. A great deal of foundational knowledge needs to be distributed among the group, and the finance expert and company management are not equipped to do this, and so initially they take a passive role as the other researchers attempt to lead themselves.

Since neither the processor nor the portfolio can sit at a table or speak for itself, their potential synchronization has to be facilitated by humans with specific expertise (Latour 2010). The array of STEM knowledge by the assembled group reflects the many areas of knowledge required to describe and interact with this computer. Whereas Galison (1996) points to how the digital computer allowed some researchers to effectively cross-disciplines by using the calculation techniques of the digital computer as a common ground for research (119), in

\footnotetext{
${ }^{46}$ Conference summary available at: http://www.quantresearch.info/JPM_CONFERENCE_SUMMARY_2013.pdf
} 
this case enabling the calculation requires a number of different disciplines to create a new cross-section of their fields to facilitate the computer.

In the first week of discussion the team begins with a general overview covering the physical properties of the chip and how its larger hardware can be expected to behave. These physical properties have already been made mobile in conversations between physicists and mathematicians at D-Wave, and disseminated through their tutorials and readings. Through their work the use of a particular mathematical model ${ }^{47}$ (i.e. an Ising spin formula or QUBO ${ }^{48}$ ) became central to any understanding of the D-Wave and quantum annealing. This model provides a reasonably reliable generalization for how the processor can be mathematically described. With such generalization accepted, researchers can then work with well known stochastic equations that are associated with these models to figure out the basics of what they would like to accomplish. As the 1QBit group tackles the tutorials that explain this together, those with a physics or engineering background have an immediate edge and tend to lead the discussion, though they often disagree over the implications of how the machine computes beyond the simple tutorials that deal with well known and easy to model problems such as the infamous traveling salesman..$^{49}$ It's not clear to the rest how the machine actually works and getting them up to speed means finding

\footnotetext{
${ }^{47}$ A translation process I describe elsewhere.

${ }^{48}$ Quadratic Unconstrained Binary Optimization.

${ }^{49}$ The problem is typically formulated as: Given a list of cities and the distances between each pair of cities, what is the shortest possible route that visits each city exactly once and returns to the origin city? Each additional city increases the computational complexity of the problem.
} 
new ways to describe its components. In these first exchanges the physicists do their best to estimate the machine's favorability to numerous concepts and techniques brought up by the team: monte carlo methods, boltzmann distributions, and markov chains all get tested for their compatibility with the embodied Ising model that is a quantum processor. The meetings range from 3-5 hours, with very few lulls. 1QBit provided laptops to work on, but all but one of the group takes notes by hand, starting what becomes large piles of notes taken on graph pads. It's easier to draw diagrams and write on graph paper. Little becomes resolved, instead, a long list of areas of uncertainty is being identified.

By the second week the team is poking holes in the provided tutorials. Their questions are quickly moving away from asking how things work, to asking why do they not work another way. Some of these next stages of questions center on the material qualities of the machine, for example: are qubits lost whenever the machine is calibrated? Are there methods for error correction? Can noise in the system be re-purposed in some way? Noise in theoretical quantum systems has always been a significant challenge as manufacturing qubits imperfectly can ruin their potential for information processing (Klesse and Frank 2005, Lanzagorta and Uhlmann 2010). ${ }^{50}$ As such challenges become the focus, the physicists start to lose a clear position of leadership in the discussion, and now are on more

\footnotetext{
${ }^{50}$ Each tiny error in the material manufacture of a qubit, or its containment system, creates a little 'noise' in the data. As more qubits are added the noise increases until it compromises any possible answers coming out of the system. Though tactics for "error correction" to address some of these challenges have been proposed it remains a fundamental challenge for quantum computing (see: Landauer 1996, Kalai 2006, Nielsen and Chuang 2011).
} 
even turf with the engineers, and in some cases of information processing questions, the mathematicians as well. The back and forth frequently requires one of these disciplines to explain a faulty assumption another has about the computer's likely abilities, which frequently lead to spirited arguments that test how many people can talk at once and still converse. These are often diffused by derogatory jokes about each other's academic fields in a tone more expected of arguing sports fans. What cannot be agreed upon, even after extensive debate, are put aside as questions to be brought up later with D-Wave's team. At this stage, the legal relationship between D-Wave and 1QBit was not fully established. So while D-Wave was willing to provide support in many material and immaterial ways, 1QBit did not have full access to their research or personnel. As their relationship became more formalized, as discussed in the following chapter, many questions that could be answered still were not, for reasons of intellectual property. Depending on the sensitivity of information or difficulty in resolving these, they could become open questions for many months, leaving the team to operate in an ongoing condition of uncertainty. For example, it was not clear if as the next generation chip added more qubits it was planned to have the same layout until the following year. An extremely important open question was the cause of a short lag in the machine communicating answers, was this from the machine itself or D-Wave's online API? This relatively short two week period of gathering such basic information about the machine and identifying what could not yet be known about it, did not have a definitive endpoint. But with a baseline familiarity of the computational system in play, the 
domain of finance began to enter the discussion and guide the direction of conceiving, investigating, and problem-solving the technology.

\subsection{Quantum for Quantitative Finance}

It is worth noting that in the group, the finance expert is, on top of not being a graduate student, actually a professor. This dynamic, combined with the far smaller overlap of the others' fields into finance, temporarily transitions the frequently argumentative discussion into a series of lectures-a known risk of professors. The following few days became an overview of numerical engineering, introducing new actors to the group (e.g. bonds, commodities, exchanges, portfolios, derivatives, futures). The finance expert especially wants to speak of the behaviour of options: how they are traded, their variations (i.e. European vs. American), the critical variables in each, and the daily normative practices of the organizations and individuals that manipulate them. By the third week of discussion entire days are being devoted to the finance expert lecturing. Some of this is running though the math used to deal with finance's actors/actants, but each mathematical operation only makes sense given the many non-mathematical constraints that exist in the techno-social-legal practices of that world. This is quickly apparent as the researchers are confused why certain obvious-seeming mathematical reductions are not being performed. The mathematicians are increasingly being turned to as a source of authority in the discussions. Each actor introduced by the finance expert is presented to them for evaluation, or they often turned to for a final word in an argument about a new actant's usefulness. For example, whether typical mathematical operations used 
in a trade are likely to be able to be converted/reduced to the equations used to program the AQC. If they suspect that object can be configured to match what the engineers and physicists described, they explore the idea and bring it back to the next discussion with an appraisal. As ideas around these objects begin to form these appraisals may happen just by thinking about them, other times some calculations may be in order.

Programming is now used to test out ideas developed in discussion. Implementing ideas in code, even in quick prototyping languages familiar to most of the team such as Python, takes time. In response, long discussions are less frequent, and start to describe the results of such experiments. Group conversations switch to a couple hours each day, then every couple days, and soon they are not scheduled at all, but happen as needed and not always with the full group. These experiments are pursuing several different ideas for how to describe the finance actants of trading. Their results are compared with small presentations or demonstrations at the less frequent roundtables. Now that coding is involved, the researchers tend to work in pairs or small groups, usually with at least one of the mathematicians. These are not assigned projects, but temporary teams exploring shared ideas and interests generated by the discussion so far. Some of these early projects aim for complex applications, such as a binary neural network that attempts to take advantage of the speed-up offered by an AQC to train neural networks fast enough to solve the real-time changes in stock dynamics. Others try to use graph theory suited to the AQC to 
map patterns of high probabilistic stock correlations to predict sudden market turns. Most of these will soon fail, and be abandoned after their prototypes do not survive group critique. Others will seem to fail but be revived once other problems are addressed. Working hours are becoming varied and desks becoming personalized. Extra shoes, pictures, and piles of paper are left behind at all hours. The flow of work has shifted from a lecture series to creative production. However, it is not randomly creative. The lectures have given the teams ideas of what the company considers important financial objects, and building anything quantum-based in their vicinity is encouraged.

The finance expert hopes to find a discrete job that requires lots of computational work, and ideally does not already have a perfect solution, which the mathematicians can put in the general mathematical format that the quantum processor is known to solve. They are looking for what $1 \mathrm{QBit}$ management occasional refers to as a "golden egg" problem; a problem that can be solved which is of such high-value it would in itself justify the existence (and sale) of the quantum computers produced by D-Wave, the software 1QBit would create, and ultimately perhaps of $1 \mathrm{QBit}$ as well. Using neural networks or graph theory to better predict markets would certainly qualify, but these are long-term and potentially impossible projects. Still, the mere possibility of these working makes them worth exploring for a significant period of time, and the glow in the eyes of the CFO is palpable when discussing their potential. The entire company is ready to entirely focus on any high-value AQC 'killer app'. 
Due to its similarity to many other finance problems, an early success in portfolio optimization would be ideal. Management imagines that as the machine and its software is constructed, it can be further tailored for such finance problems. This vision of the computer as a highly specific tool for a small number of industries is a common view across the two companies. In this way, quantum computation and quantitative finance may become linked industries and grow together, as finance and trading have with the incorporation of other computation and communication technologies. Everyone is highly motivated. They have their work cut out for them.

\subsection{Applying Imperfect Tools}

D-Wave already has a method for achieving what the team is trying to do, finding a way to translate different kinds of problems into the native form for the processor. A piece of software, called "Blackbox" has been provided to run nonnative problems on the processor. However, in order to do so, Blackbox does not really represent the problem accurately and it does not really solve it optimally, which is the entire appeal of the AQC method of computation. Instead, Blackbox uses a series of approximations to create an inefficient embedding on the chip. Exactly how Blackbox works is not disclosed to the team, but after trying out various known problems its limitations become clear. It will not provide solutions better than a digital system and it tends to do worse as the number of variables increase. The team hates it, and jokingly started calling their project "whitebox" in 
response ${ }^{51}$ Finding a better way than Blackbox is tied to the goal of finding a particular new finance solution as it becomes clear how badly Blackbox handles solving such problems. By the end of the first month of the roundtables, the team finds that instead of using it as a way to translate problems, Blackbox serves as a good way to test how non-native a problem format would be to the processor, and compare their own translation methods to D-Wave's standard. It provides some guidance on how a QUBO is best written for the processor, but serves more as an example of what not to do that could be repurposed as a type of baseline test of problem-structure. This transforms the intended purpose of Blackbox, finding a new role for it in the quantum computing network that benefits 1QBit's emerging vision of that network.

Similarly, D-Wave created a simulator for the quantum processor that they distributed to research partners such as $1 \mathrm{QBit}$. The simulator is a digital application run on a digital computer that mimics the behaviour of the quantum processor for solving problems with a relatively small number of variables. Quantum computing simulators ${ }^{52}$ are not uncommon in physics and computer science research, and though they have many limitations are considered useful tools for exploring the numerical relationships of highly entangled systems. ${ }^{53}$ While there are many issues that make the results of the simulator only

\footnotetext{
${ }^{51}$ Sadly this name did not stick as projects were later formulized.

52 These differ from quantum simulators, which use actual quantum phenomena to model systems for physics experiments, such as proposed by Feynman (1982).

${ }^{53} \mathrm{~A}$ list of quantum computer simulators, and detailed explanation of their uses can be found at: https://quantiki.org/wiki/list-qc-simulators
} 
provisionally reliable compared to results of the actual quantum computer, having the tools of the mathematical models and the simulator allow early ideas to be tested and in some cases ruled out. In this way, those around the table not only talk, but interact with representations of the quantum computer to "beta test" early ideas.

By the second month of roundtables, far more time is being spent at desks in pairs or individually, with the group discussions becoming moments of reporting back and setting general directions for next research steps. This is partly the case because tools like the simulator temporarily isolated the need to take into consideration the nuances and complications of the physical machine. These would have to be returned to as any idea was refined, but in the meantime they provided space for experimentation and quickly testing ideas on other actors than the researchers themselves. With the quantum machine itself made more distant by these existing software actors, the mathematicians remain the main participants in the group conversations. Yet, rather than a discourse with two sides, one financial and one mathematical, the conversation is complex and comes from many different angles. The breadth of the discussion and its participants creates difficulty for scholarly analysis using conventional methods and to represent here in writing. However, without actually running through all the mathematical steps described in their conversations, it is still possible to outline the overall act the researchers were engaged in over the course of their experimentation and discussions: the creation of a problematic. This can suggest how to think about problematics in computation, given the process of a 
problematic's construction and agency. To develop a problematic for this difficult and limited computer, the researchers need to make several transformations take place.

\subsection{Reordering Mathematics}

The translation work now required of $1 \mathrm{QBit}$ 's researchers is to take the domain knowledge of the finance expert and bring it into contact with the interdisciplinary study of adiabatic quantum computing software design, which is emerging out of this gathering of the researchers' expertise and practices. A common language exists around the table to help this, mathematics. But the dialects of mathematics are diverse and aligning them is not a simple process. This is especially true because it is not purely abstract mathematics ultimately being spoken of, but material actants that occupy very different social worlds and occasionally break away from their models or previously established practices. Qubits especially do not always behave as they should, returning errors or worse, degraded solutions. The order and techniques of mathematical translations does not come into quantum computing with a preset system that can be used to solve new problems easily. The kind of optimisation the quantum processor specializes in, linear optimization, would not be a typical goal for a mathematical translation. Instead, as the below exchange with one researcher explores, the 'common sense' of mathematical practice is impacted and rearranged:

Researcher: In mathematics, whenever you want to solve a problem, this is the way usually mathematicians work: they solve a simpler problem, then they look at the harder one and then they try 
to reduce that to the simpler one. It never has been the case that, when you do optimization theory the simplest question you answer is linear optimization and then when you want to do other things....when you do quadratic programming, you also reduce it to some linear programming in the end. Eventually all of them...you do something of the simplest type [linear]. Never in continuous optimization has anyone tried to reduce it to mixed-integer optimization. A mathematician will never think of a mixed-integer optimization problem as an easy one. We [now] have this assumption.

Me: So it reorders that logic?

Researcher: Exactly, like, everybody who does integer optimization they talk about continuous relaxation, because continuous problems are easier when you have enough structure in the problem, then you try to reduce the integer problem to the continuous ones. Now we [at $1 \mathrm{QBit}]$ are going backward, we are assuming the integer optimizations are easy, and now we are looking at hard continuous problems and try to reduce them to integer optimizations. So, it's because of existence of this machine we have a new assumption that something can be the building block of algorithm, and then we are reducing problems to that 
one.... whatever technology you have effects the models you have and that effects what mathematics means. [emph mine]

Mathematics is a tool that can be used to make these worlds translatable, but it does not provide a ready-made solution that can be integrated into software. Mathematics here becomes far less abstract and fully entangled in the technical and social requirements of its application.

\subsection{Creating and Simulating New Problems}

At the center of the team's conversation is the mutual need to establish a new problem. This problem must be compatible with both the social world of the sector of finance targeted by the company and the social world of the quantum computer as it currently exists, with all the problem-solving implied with working with an unstable and largely untested system. This seminar has been their negotiation point, where radically different expertises are finally being mingled at a granular level to see if one should care about the other. Success at this point guarantees nothing except the continuation of the process to connect these social worlds. For a new startup such as $1 \mathrm{QBit}$, however, that temporary further growth is a major goal. Without the short-term goal of the company, the team would not have been assembled and given a direction to investigate. The solution they are seeking is the creation of a problematic particular to this context: a new view of the valued objects in finance which aligns with the 
computational patterns of qubits. ${ }^{54}$ The problematic would need to expand not only the burgeoning practice of quantum computing, but also to create a space in the otherwise 'full' field of options trading. Traders have not been seeking a quantum computer, rather, establishing a problematic is the step needed to make quantum computation a "matter of concern" (Latour 2004) to these currently unconcerned traders and financial institutions. ${ }^{55}$ There is very little common ground to start from. Though the finance expert and mathematicians had initially tried to speak to each other in mathematical terms, simply sharing sets of equations isn't very useful for creating a new problematic. The problematic is only partially a mathematical device, more broadly it is a reordering of the world that breaks apart existing programs of action. A new problematic explores unused agencies of actors in a network, and repurposes those already in use. Putting such changes into place requires the team to problem-solve constructing all these new relationships in a network. The resulting problematic itself results in a new program that filters the world for problems that fit this newly identifiable shape.

Building the connections between the actors of these initially separate networks begins with many misunderstandings and introductions. When the mathematicians are introduced to a series of common trading algorithms the response is, at first, deceptively positive. It all makes sense to them and they ask

\footnotetext{
54 In this case, the behaviour of supercooled niobium that is put into a quantum state.

55 Perhaps more accurately, further concern, as some financial institutions such as Goldman Sachs have provided some investment in this hardware.
} 
why other types of mathematical reductions are not being used. However, before hearing more, one mathematician hesitantly asks the question, "But...ok, can you explain what a stock is?" At this question, there was a sigh of relief from the majority of those around the table, as they also were struggling with understanding the basic existences of many of finance's fundamental actors. The 1QBit researchers do not need to fully interact with the real objects of finance. They are not going into finance themselves, but rather seeking to create relationships with those who do. What they must translate into the quantum computing network is not the behaviour of a real stock, but the common representations of stocks. Before the finance experts' leading of the discussion comes to a close in that first month of roundtables, they therefore have moved on to speaking less about the real world and more about the translations and representations that pass as the real world in finance.

\subsection{Good-Enough Modeling and Techniques}

The finance expert needs to represent the world of trading, banking, financial services and consultation, so that the researchers can have a working understanding of that world, with the added complexity of trying to have them help to suggest points of disruption around which a new problematic can be formed. This requires an introduction to the theoretical object of the market, specifically option and portfolio trading with their attending mathematics. Luckily there is a well known mathematical model of the market used in this area of 
finance, the Black-Scholes model. ${ }^{56}$ Transforming the formulas used by BlackScholes to be more compatible with the QUBO format that represents the processor provides a new goal for the team to pursue. At first glance this appears as a common sense and potentially fruitful direction. Black-Scholes is already a format for kinds of optimization, so aligning the two is plausible even if they do not convert precisely. The mathematicians are excited for possibly the first time in the conversation, these kinds of conversions are familiar territory for them. If it is just a matter of balancing penalties and constraints this seems like a workable translation. However, the finance expert is quick to point out this model does not actually reflect the assumptions and practices of those who do real-life trading. The discrepancies between the Black-Scholes model and the world are also well known. For example, the finance expert explains that unlike their depiction as a formula, aspects like interest rates and volatility are not expected to act as constants by practitioners. Black-Scholes may provide some insight, but a more concrete practice is needed for the group to focus on. The conversation moves on, first, to what would be useful for traders that does not exist already, and second, out of what does exist, what is considered computationally intense?

Computational intensity is not an objective measurement. Deciding what qualifies requires assessing what is considered good-enough computation. The expectations of what can be computed to affect a trade decision, including speed of communication, available information, and prediction techniques all factor into

\footnotetext{
${ }^{56}$ Black, F and Scholes, M. (1973). The Pricing of Options and Corporate Liabilities. Journal of Finance, 637-654.
} 
what establishes the norms of good-enough computation in finance. As new technology or information processing strategies become available these norms have changed to various extents, creating new expectations for speed, relevant information, and participation in trading with hardware and software upgrades (Black 1971, "Effects" 1984, Carey 1989, Cassidy 2002, Flinders 2007, Kim 2007). Several days follow of the team discussing, frequently argumentatively and without clear consensus, what kinds of previously computationally difficult variables that the new computer probably can tackle would look like. It becomes clear that whether the quantum computer can handle, let alone improve upon, current financial problem-solving will not be known until potential solutions are tried and quite far along their development. After several days of this the finance expert finally determines an initial problem for the researchers to try to solve with the quantum processor: the optimization of a portfolio of spread options. Spread options are a common type of trade that deal with predicting the relative value between two stocks. Typically this compares stocks that have historically had correlated values, and for any variety of reasons are expected to continue to be predictive of one another. In most cases, the reason for their relationship does not have to be known or even theorized, but if the relationship is taken into account it can help balance large portfolios. To help convince the team that this would be a ripe computational target, the finance expert explains the limitations of how this problem is currently handled by practitioners in quantitative finance. One technique for simulating the future behaviour of such options involves creating a binomial tree (see Fig 2). 
The binomial tree is a well known exhaustive computational approach that creates a prediction for each possible path of a stock at various future points in time.$^{57}$ Similar predictive methods are used in many other contexts than finance. The researchers recognize the tree structure and its logic from different applications in their own fields and understand its challenges, though each of these contexts has a different language and nuances in application. Calculating such a tree strategy for large portfolios of interrelated spread options, particularly when adding additional variables at certain points in the tree, certainly qualifies as a computationally difficult task to the point it cannot be applied to larger portfolios (e.g. those found in energy trading) with currently available digital computers. Exhaustive approaches in general are poor tactics for problems such as optimization, because the number of potential solutions an exhaustive search must go through can grow exponentially with every added variable in a problem. Finance has many computational problems of this magnitude; however, it also has developed reasonable solutions that can be created in a short amount of time for these problems in the form of heuristics. Heuristic answers/methods (e.g. monte carlo algorithms and simulated annealing) solve these kinds of optimizations, but never provide an exact optimal answer to the problem. In the terms of the energy landscape introduced in the previous chapter, they arrive at minima or maxima which are not global minima or maxima. Meaning that better answers than the ones they produce could exist but they are sub-optimal. Instead

\footnotetext{
${ }^{57}$ This requires creating certain constraints around the prediction of the tree, such as the amount of difference that can be created at each step of the tree and a relatively arbitrary creation of discrete times to measure/predict the stock's behaviour. These considerations construct a notion of the market around convenient mathematics and calculability rather than real-life phenomena.
} 


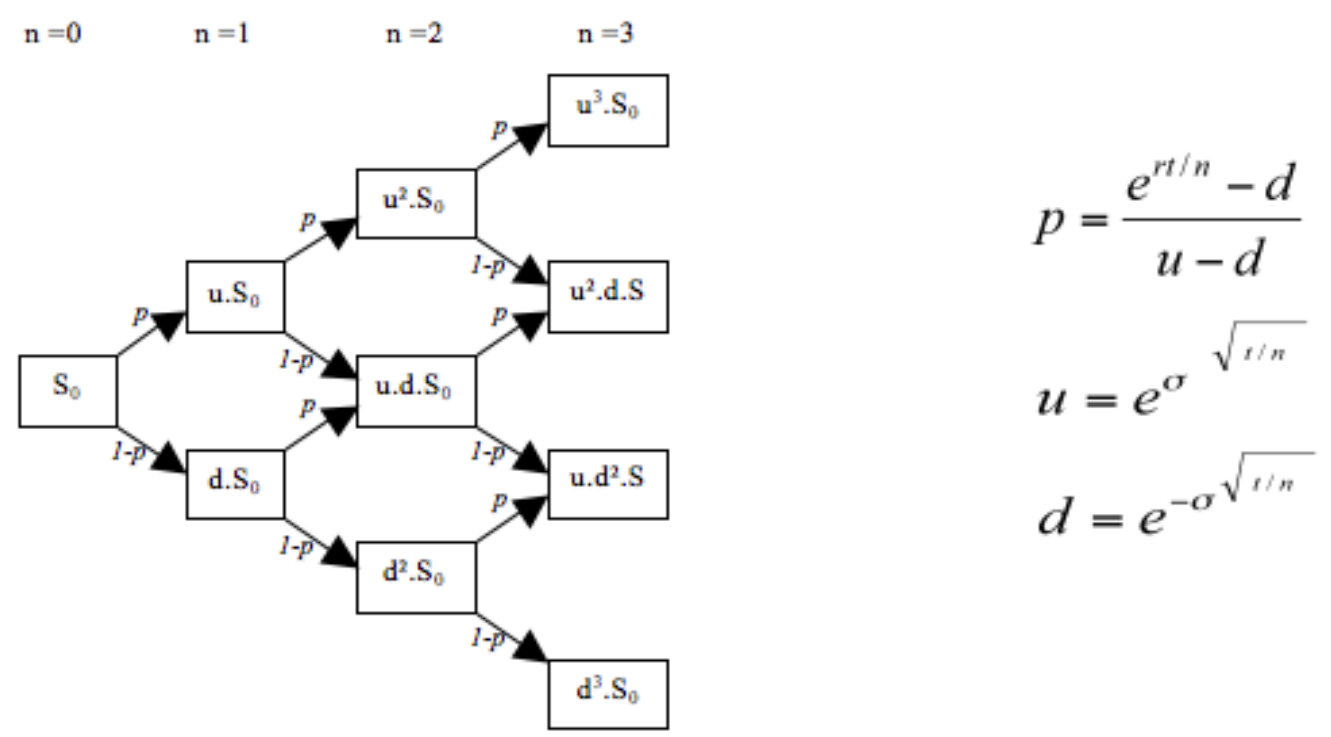

Figure 8: A binomial tree. Source: Wikipedia Commons. User: Joly-Stroebel.

of finding the best answer they use probabilities and sampling strategies to arrive at a good-enough answer for the asker; a criteria set by the expectations of the user, which in turn are normalized by the possible performance of available technology. These heuristic strategies were pioneered early in the development of the digital computer precisely to address the inability of digital computation to deal with problems that grow exponentially with additional or larger variables (Galison 1996). In some cases, the difference between a good-enough and a truly optimal answer may be valuable. In other cases, it may be insignificant. The benefit of a quantum computer to these problems is that it can provide trueoptimal answers (i.e. global maxima and minima) which may create substantial value in the high-stakes margins of options trading. But whether meaningful value, by the arbitrary standards of traders, could be created is unknown until a problem can be transformed into the understandable mathematics for the 
quantum processor's qubits and compared to its conventional equivalents used by practitioners. In this, the D-Wave simulator plays an important role. In the early stage of $1 \mathrm{QBit}$ 's research, the quantum processor was not always available, due to maintenance, the process of establishing a secure online access, or the need to share it with other research organizations. In contrast, the simulator could be used on a laptop with no security protocols and was an immediate way to test various 'hunches' in the research process quickly ${ }^{58} \mathrm{As}$ a digital application, it was also an easy method for the researchers, who were nearly all competent programmers due to the methodological needs of most STEM disciplines, to test the validity of an algorithm (i.e. the Black-Scholes converted to a QUBO) before fully considering the requirements to create quantum-based software.

After such experiments with the simulator, the researchers have bad news for the finance expert. At least at first. The complex portfolio optimizations for option trading cannot be represented in a way compatible with optimizations used by the quantum processor. ${ }^{59}$ For this problem, despite the motivation to use it as a proof of concept for the superiority of quantum annealing over digital methods, it cannot improve on the current solution. Even though it provides optimal answers and digital computers only pretty-good ones, to be computable in this format and

\footnotetext{
${ }^{58}$ In this way the speed of the digital organization (including laptop, people, etc) was able to still surpass the far faster computational time of the actual quantum processor for small tests.

${ }^{59}$ A series of graph theory conversions are required for any problem to be solved on the processor. With current limited chip size, the available graph is simply too small for the complexity (number of constraints) of a portfolio.
} 
on the current chip it could not include all the data from the problem and became even less true to life than the Black-Scholes attempt to model market behaviour. However, what about a "toy-problem" that greatly simplifies the objects and their constraints being computed? Or even part of a toy-problem? Through a series of concessions away from the annoying complexity of real-market behaviour, they suggest and iteratively test on the simulator whether it is possible to model a simplified portfolio optimization. Having lost the expectation that the quantum processor could currently solve the full problem, they propose to run it not as a single problem on the quantum computer, but as a series of smaller problems broken apart. The principle of segmenting large difficult mathematical problems into subproblems and solving those as a step to find the full answer is referred to as a "divide and conquer" methodology. This phrase was repeatedly used by the researchers to describe how they were approaching this problem and many others. The term is commonplace in STEM fields, particularly mathematics. However, in this problem it became not just a model for problem solving, but for software application design. The divide and conquer approach created a subset of problems that through simulator-based experimentation could be identified as parts that could be solved effectively on the quantum processor and others that would be advantageous to be solved/pre-processed on classical (i.e. digital) computers. Initially this made sense as a compromise to try to show that this particular portfolio problem could to a degree be tackled using quantum annealing technology, with the premise being that a larger processor would one day be able to process the entire problem. However, once established, the idea 
of a hybrid quantum-digital computational model became a more real reference point for further development than the idea of a future chip.

\subsection{Negotiating Hybrid Models}

The team was able to spend the next month programming and benchmarking the results of this pseudo-solution, but several of the researchers were not sure what to do with the project. Even with the reworking of the options trading problem into this divided hybrid model, the researchers were still left with a problematic/solution that had no real-world applicability. It is not something a quantitative finance expert would ever use. Its results could not improve upon the state-of-the-art. But does the problematic have to be an improvement, or even work? Not by the standards of the finance industry. Despite being industry-driven applied research, effectiveness was not ultimately the measure of success for software resulting from these roundtables. The notion was to create a relationship through a problematic, not a solution for a current portfolio. This can be seen in how developing the simplified portfolio optimization ultimately worked based on a computational compromise rather than the intention of demonstrating an entirely new quantum paradigm.

With the simulator showing promising test results for a simplified (i.e. inapplicable) way of modeling/converting the problem, the group began coding the algorithm to be run on the quantum processor, and subsequently refactoring it to run as quickly as possible. Even though the processor itself, as mentioned above, is "programmed" by the turning on and off of various magnetic fields 
instead of running commands on a processor, applications still can be encoded in common programming languages. Python and $\mathrm{C}++$ are typical choices, for their easy prototyping and speedy execution respectively. This is due to the operation of the quantum processor's magnetic switches being controlled by a digital computer attached to the side of its chamber for input/output purposes. In this way, the hybrid algorithm can be coded using the familiar tools of digital computers, but the functions/algorithm coded only make any sense given their ultimate execution in a quantum-based system.

The resulting hybrid software created from this conversation series was first labelled as the Portfolio Optimization Toolbox. It can be said to solve a portfolio optimization with a small number of assets using mathematics native to the quantum processor (i.e. the problem of minimization of a low degree polynomial over an integer lattice). With an early description provided to JP Morgan from when the application only had been tested on the simulator, 1QBit secured a presentation spot to appear in London, UK to describe the approach four months after the roundtables began. When the hybrid-algorithm was encoded for the quantum processor it ran as predicted by the simulator's projections, which is to say, it accurately predicted the quantum-software's mediocre job of sort of optimizing the portfolio. However, its reception at the JP Morgan conference was notably positive. This surprised the researchers who had devised it and were quite concerned with its technical limitations, but 1QBit's management did not foresee the same criticisms as they were evaluating its use quite differently. As the finance expert described their reception at the conference, it succeeded 
simply because it "got them thinking". Later that year, one of the world's largest trading organizations, the CME group, became 1QBit's single largest investor after being introduced to 1QBit's work through the JP Morgan conference.

\subsection{Expectation Versus Emergent Solutions}

The desire of the finance expert to solve his example trading problem was never met. Neither were the hopes of the researchers to find a real-world problem that could be directly mapped onto the qubits for a pure-quantum solution. But methodologies were established and the network of interests trying to connect to these qubits grew. The encounter brought quantum computing into the world of finance practitioners, and gave them the impression that this was something that affected them and their world. It enlarged the sphere of quantum computing by entangling these new actors and their respective networks.

The hybrid quantum-digital problematic, and their attending integer based mathematics deployed for the portfolio optimization, that developed in this conversation and ensuing toolbox became a default method for 1QBit to engage with other real-world problems far afield from finance (e.g. oil drilling, molecule comparison, Job-Shop problems). It also eventually lay the groundwork for 1QBit's first software-based patent. This connected and extended the software through a number of legal actors (e.g. patents, lawyers, databases), described in the following chapter. Interestingly, as the idea of the toolbox was refined through more such mobilizations it became increasingly distanced from its finance roots. Early drafts of white papers and 1QBit's ensuing patent of this approach 
remained centered on the trading problem. However, successive iterations of both documents (and adjustments to this hybrid-method algorithm) were renamed as the "Integer Optimization Toolbox". This gave the technique a potentially broader enforceable legal protection and shifted the resulting software further from an "application" and toward a "system" software function, settling somewhere in between.

This example shows how the computerization of new materials and architectures in quantum computing became a motivation to reconceptualize the trading interactions of markets. This effect was reciprocal, as the existing agents that make up the market led to the development of software/algorithmic approaches as a response to the pressure to impress and involve a lucrative industry by a research and development company. This does not turn this emerging new kind of computer, however, into merely an exotic 'trading device'. The computational architecture may not be general purpose to the degree of a Turing machine, but neither is it single purpose and committed to the influences of the industry its lowest-level software has been constructed around. The attempt to find a working problematic is about creating social relevancy that allows the network of quantum computing to expand into new territory, and gather new resources to expand further. That relevancy can be generated in a number of ways, drawing on different acts and their effects to establish credibility, authority, and legitimacy in new domains. 
Overcoming the limitations of the computer requires finding new ways to represent problems through software development. Software also must similarly overcome or accommodate previous technological solutions and their established institutional norms. The passing on of commands through the levels of coding languages that exist between an end-user and a transistor in a classical machine have established one way for problems to be run on a computer. Quantum computing, however, draws attention as well to the mathematical translations being done directly by humans to connect phenomena in new ways. These translations are largely outside of the machine, and while they are at times materialized in the software of particular software toolboxes or protocols, they also form a paradigm of computation by establishing decision making processes and logics.

The development process of the software described in this chapter, in its techniques, goals, and results, is quite different from the digital norms of 'appdevelopment'. Instead, they follow a process of "connecting the real-world to the machine-world" ${ }^{60}$ This connecting process in digital computers has largely been tackled by the development of systems software, and what falls under the human-computer interactions facilitated by an operating systems. The team regularly described this connecting process as the work of building up a new "paradigm of computation".

\footnotetext{
${ }^{60}$ A phrase proposed by one of the engineers in the roundtable to describe their work.
} 
This connecting work does not put software between the layers of hardware and user, but shows software as deeply entangled with the structures and interests of institutions as well as the "machine-world". This practice emphasizes the process of building and hybridizing worlds. Instead of an isolated layer, software becomes a series of negotiations between many kinds of actors with different ideas about what should result. In this process of developing a computation method through the connection of these heterogeneous actors, establishing a problematic enables and creates limits for a pattern of interaction for new actors to be computable, in which the early actors are highly influential but not determinative. The problematic itself is a way to encapsulate the methods of distribution available to computation. Rather than being a well bounded category as might be implied from the description of the move from a digital to quantum paradigm in computation, the development of a problematic shows construction through negotiations with the material, social, and institutional agencies broadly at play in computation. Despite its mushy sounding name, software tends to last longer than hardware (Ceruzzi 2003, 151). As quantum hardware and applications invariably evolve, the decisions that come out of software development may carry forward as they entrench particular problem-solving perspectives into their networks. 


\section{Chapter: From the Quantum Computer to Quantum Ready: Distributing a Mindset in an Uncertain Actor-Network}

At a World Economic Forum panel in June 2016, titled "Betting on Moon Shots", 1QBit's CEO, the President of Baidu, the President of the European Research Council, the Director of the Robotics Institute at Carnegie Mellon University, and the President of Rensselaer Polytechnic Institute discussed the new normal of corporate led scientific research and whether it helps or hinders scientific advance. The panel assumed that the idea that academia conducted basic research, and corporations only applied research, was increasingly out of date. They argued new and varied collaboration models are increasingly the norm, and create many advantages and challenges. This transition has come out of the recognition that basic research is often what creates sudden advances in new technology, and the desire for corporations to be, as 1QBit put it, what "catalyze people around a specific purpose". The original eponymous moon-shot was catalyzed as a government project, and drove basic research funding and its transition to technological developments, creating many unplanned technologies as well as those required for space travel. However, the moon shots of lofty goals like strong A.I. or universal quantum computation, take many incremental steps that are not always of interest to corporations, and can move the technology in unexpected directions. As $1 \mathrm{QBit}$ describes itself as a catalyst providing a trajectory to technological development, it takes on the role ANT literature describes as a spokesperson arguing for a vision of an actor-network with which 
other actors can align (Callon 1986, Law and Hassard 1999, Latour 2005). But 1QBit does not know what the actor-network or itself will be in advance. They must develop an internal mindset that accounts for the quirks of the technology's incremental steps, and find ways to make this mindset external and shared through the quantum computing actor-network. Focusing on a mindset calls attention to the development and negotiation of mental frameworks that must now accommodate new socio-technical actors, guiding how human actors exercise agency in a network. This guiding can come internally, by adopting reference points and expectations for the network and the actors' role within it. It can also be generated by organizational forces, slogans, assignments, memos, or job and project descriptions. Mindsets allow the network to be translated into larger abstractions, inspired, but separate, from particular technological solutions or material actors. They shape the choices made in defining the network and growing into the broader social world.

Mindsets do not have as much flexibility as descriptions of social constructions. They must allow socio-technical work to be performed, and so retain an empirical quality. Rather than a depiction of a naturalized state or force (Barthes 1972), they merely claim to show/enable what work is possible to program with a particular configuration. They are a process and effect of trying to stabilize and coalesce local socio-technical translations into more generalized organizational tools. This can involve retroactively re-imagining how the network has formed/functioned in the past to create a coherent sense of its future trajectory. 
Unlike a stabilized ideology, the mindset may evolve quite rapidly to enable new translations/work to be accomplished, and is not entirely produced by human actors (Macey 2000, McLellan 2007). This translates the skillsets, material developments, and aspirational visions of the network into a coherent narrative that can help it grow in a particular direction. It contains the known possible actions that the network can be programmed to execute (Latour 1992).

The quantum computing mindset at 1QBit differs from those of other groups who conceive and configure quantum technology in alternative arrangements. For example, the goals, technology, and relevant actors outlined in the Quantum Manifesto published by the EU presidency suggest an entirely different path for the technology around other catalysts. ${ }^{61} 1 \mathrm{QBit}$ 's mindset evolves in part with the organizational practices they use to make the world computable. The work of information and categorization practices is conducted by those working with machines and problematics, but also as those processes are circulated and added to, as they are made meaningful to the outside world's other actornetworks. The translation process involves finding a place and identity for the network of material delegations and human work that make up each new actor in quantum computing. Just as the qubit emerges through a translation process and is given an identity by establishing a host of relations, so does 1 QBit. The previous chapters have addressed how technical work re-classifies and often transforms actors as it translates them when inside a quantum computing

\footnotetext{
${ }^{61}$ https://english.eu2016.nl/documents/publications/2016/05/17/quantum-manifesto
} 
system. Here I want to examine the organization of quantum computing in a way that addresses the institutionalization and preservation tactics at play in its commercialization.

The quantum computing world is growing rapidly, but it is still small. In this it differs from familiar studies of large technical systems (Hughes 1985, Kaghan and Bowker 2001, Edwards 2010). Yet, it increasingly prepares to be incorporated into broader computational networks as the "gateway technologies" required to make a multi-standard computational system of digital-quantum processes are developed (David and Bunn 1988). In a sense, it is a mediumsized technical system, with aspirations. This expansive moment of quantum computing is a search for where and how to build the bridges it needs to connect with the established computational landscape. The methods involved combine technical, legal, and corporate strategies. Examining these methods suggests as a network, it is still open and very liable to change at all levels, from the connectivity strength of qubits to the partner agreements of investors, as it tries to fit into that world. In this chapter I want to pay particular attention to the networks that are required to create and maintain this medium-moment of commercialized quantum computing, which may become less visible after its somewhat unknown trajectory proceeds. Rather than depict corporate growth and strategizing as a separate realm of decision making and agency in a formalized organization, I hope to highlight the translation work between many kinds of actors that formed, held together, and affected the course of the company. The process of creating the 'content' of the company and circulating it 
in order to grow the network, is spread throughout seemingly separate levels of the organization. It is not a case of researchers creating content and managers organizing and selling it. As Kaghan and Bowker (2001) point out, the "messy" process of growing a socio-technical system involves "invention" (i.e. creating new actors) and "innovation" (i.e. deploying those actors in new contexts), processes which are shared across many actors rather than clearly segmented. As resources are committed to quantum computing, more actors become involved in working on translations that hold the entire network together. The work done to create and maintain the negotiated orders of a socio-technical system is perhaps never complete, but valuable to see how it has been achieved so far.

\subsection{Quantum Actants in a Classical World}

How to describe and classify the participants and products of the quantum computing industry is an evolving and contentious practice. As organizations become involved in an industry, their positions and resources are distributed through a broad range of ongoing infrastructural work. As 1QBit works to expand the actor-network of quantum computing, bringing in new people, organizations, technologies, materials and knowledge, the work to connect with and classify each has numerous repercussions. Controlling the flow of actants and agencies of an entire industry is impossible, but techniques can constantly be deployed in a shifting landscape to gain a favourable position. The struggle to do so shows familiar logics and approaches in a socio-technical construction, of capitalism's approach to scientific production, the use of technical documentation and 
partnerships in business strategy, and the shifting of identity and goals that results from the dynamic co-construction of technical and social actors.

Considering this allows us to question in what ways the quantum industry is different from any other computation business. The methods used within it to open up and close down flows of knowledge are crucial to how the technology is taking shape, but these actions and agencies are not unique to quantum computers. Because they are not unique, examining them shows how new exotic actors are constructed out of the familiar, and made to fit into the already operating world.

The early development of commercial industries has often relied upon subsidized funding from government programs and the adoption/privatization of basic research conducted in academia (Dyer-Witheford 1999, Mirowski and Horn 2005, Markman, Siegel and Wright 2008). The previous chapter examined how basic technological research is not isolated from the business relationships and planning in which it occurs. In the production of many new technologies and theoretical discoveries there is not a clear separation between academic and industry research and development. However, at this moment in the development of quantum computing, there is a conscious effort to translate the objects of an academic field of research into actors for a commercial industry, which requires the deployment of many techniques to materialize previously academic-based knowledge directly into commercial property. 
Several narratives could be offered as to when the quantum computing industry began. Should the annealing camp of computation fizzle out, then undoubtedly descriptions of ion-trapping technology in the 1970s and the commercial fabrication of quantum-based materials might be looked at as a starting point. Likewise could the development of quantum algorithms by commercial researchers through the 1990s, or, IBM first deploying parts of those algorithms in 2000 on a simple 2-qubit system. These important preliminary steps in quantum-based technology and computation differ, however, from the attempt to commercialize and deploy a full quantum computing system for profit, as part of the practical computing world. Taking that as a standard, the industry may have been created at the incorporation of D-Wave in 1999, its first prototypes in 2007 , or initial sales in 2011. It was as this point that the company declared it had "the world's first commercially available quantum computer" - much to the annoyance of the many academics working in quantum computing departments who did not consider an annealer a computer, or identifiable as quantum.

D-Wave developed early relationships with corporate clients (i.e. Google and Lockheed Martin) that helped it gain legitimacy, at least in the eyes of the business world and news media (Dillow 20111, Thomas 2011). It also developed associations with many university departments in Canada and the USA to encourage research in their version of quantum computing. In this chapter, however, I focus primarily on $1 \mathrm{QBit}$. 1QBit itself represents a move to grow an industry beyond D-Wave and its initial users, and a great deal of the company's projects reflect this. Following $1 \mathrm{QBit}$ shows an actor struggling to make itself an 
obligatory passage point in a new network, for whom the identity of actors is a problem to be solved (Callon 1986a, 1986b). To solve this problem, 1QBit works to develop and nurture a new mindset for quantum computing that attempts to resolve discrepancies between the many visions of quantum computing and its current state of being. Attempting to navigate this position/process requires 1QBit to engage with other organizations and institutions that will help it infiltrate, undermine, and reshape the industry of quantum computing.

\subsection{Creating/Enclosing the Quantum Commons}

On April 15, 2016, Prime Minister Justin Trudeau visited the University of Waterloo to announce $\$ 50$ million in funding for the Perimeter Institute for Theoretical Physics (PI). In what became something of a viral moment, Trudeau excitedly described the possibilities of superposition and qubits for quantum computing, had a skype with Stephen Hawking, and argued that this research center would help secure Canada's position as a leader in quantum computing. Though clearly rehearsed and followed by a minor media backlash for its orchestrated nature, this funding announcement reflected a growing expectation that quantum computing is recognized as having an ever broader cultural and economic impact. ${ }^{62}$ The thrill with which many tweets, blogs, and articles initially celebrated the apparent quantum fluency of the Prime Minister certainly reflect the technology's growing name recognition. For many it is a flagship futurist technology, making its investments seem forward looking and exciting. Trudeau's

\footnotetext{
${ }^{62}$ IQC quickly followed with event such as "Now you too can talk Quantum!" promoting a general literacy in quantum computing for laypeople.
} 
announcement actually did not reflect a sudden shift in government funding priorities, but the continuation of an ongoing investment by provincial and federal governments in the Perimeter Institute ${ }^{63}$ and its approach to quantum computation. The Perimeter Institute's work contrasts with D-Wave and 1QBit's in many ways. Most fundamentally, the Perimeter Institute researchers largely explore information theory aimed at a general purpose gate-model quantum computer, as opposed to a quantum annealing architecture. These different architectures in turn suggests different problematics and applications as being central to quantum computation. The Perimeter Institute will not likely be able to develop a working or commercializable machine for many years, possibly decades, but this isn't their goal. As numerous machines become closer to being realized, such investments signal an intention to compete in the industry that will form around them, creating areas associated with general quantum computing expertise. In Canada, this has been split between D-Wave and 1QBit in British Columbia, with strong ties to UBC and SFU, and the "quantum valley" in Ontario. The notion of the valley is to create a "Toronto-Waterloo corridor" dotted with quantum computing expertise, ventures, and investment, comparable to Silicon Valley in the early 2000s. The valley has been driven significantly by RIM cofounder Mike Lazaridis, who both endowed the Perimeter Institute and runs a "Quantum Valley Investment" fund with RIM cofounder Doug Fregin ${ }^{64}$ This center of activity supports basic research for the technology, but also aims to

\footnotetext{
${ }^{63}$ Began under Prime Minister Harper, suggesting the broad appeal in being seen supporting such research.

${ }^{64} \mathrm{http}: / /$ quantumvalleyinvestments.com/
} 
develop venture capital attention and presence in the area to help commercialize this work. Their efforts have generated more focused government financial support than D-Wave's more private venture-based funding, and reflect the many public-private partnerships funding the development of quantum computing globally. The European Commission Workshop on Quantum Technologies and Industry estimated there are 7000 researchers working in Quantum Computing around the world, with a cumulative annual budget of $\$ 1.7$ Billion USD (IDC 2015). This includes a range of government and corporate actors; for example, a $£ 37$ million investment from the UK government for the UK’s National Quantum Technologies Program; The Dutch government's research institute and other organizations investing $€ 135 \mathrm{~m}$ in the development of a super fast quantum computer; $\$ 50$ million committed from Intel to quantum computing research, as well as Microsoft, IBM and Google's own research groups working on quantum computing (Conway, Joseph, and Sorensen 2016).

The impact of government military-industrial projects in shaping the development of the digital computer has been well chronicled in histories of computation (Edwards 1997, Ceruzzi 2003, 2012), and was briefly touched on in Chapter 4. The ancestors of today's digital machines arose to solve the paths of missiles, the implosion points of nuclear warheads, and the communication systems of warfare. Massive government funding for computation projects is not what it once was, which is not to say that large ambitious software and data projects such as the dissection of metadata, encryption, and surveillance techniques by government agencies are not regular budgeted items, but that foundational 
hardware and software developments for quantum computing will be subsidized but ultimately left to academic departments and corporate actors to develop. Bridging the gap between academic experiments and materialized machines that can be put to work for a variety of corporate, public, or government agendas is left largely to venture capital. The commercialization of the internet throughout the 1990s and early 2000s created an arms race in software development that created a legacy of massive acquisitions for select software firms and IP (Cassidy 2002, Ceruzzi 2012). Even post the dot.com crash, silicon valley based companies like Google have continued the acquisition model. To be bought by 'a Google', is a common business plan for computing start-ups, even if statistically rare ${ }^{65}$ The spectre of Google could certainly be felt at $1 \mathrm{QBit}$. It was a known deep-pocket with a stated interest in quantum annealing. In the future, it may absorb the technology, but at this point it was a background presence that needed to be both impressed and defended against.

\subsection{Opportunism and Investor Privilege}

The development of 1QBit required several opportunities. It depended upon using and creating relationships between a variety of individuals, organizations, and resources. These include a variety of interpersonal and institutional connections. The founders of $1 \mathrm{QBit}$ came out of an investment group (Minor Capital) which specialized its resources toward high-tech companies that were seen as particularly disruptive to current markets. D-Wave Systems was an early

\footnotetext{
${ }^{65}$ The $\$ 500$ million acquisition of DeepMind in 2014 , an Al firm, was a frequently discussed example in the office.
} 
investment for Minor Capital, and while their investment was comparatively small next to the larger institutions from which D-Wave has repeatedly sought funding, this was an important avenue for access to the scarce public knowledge around the development of the technology between 1999-2011. Being shareholders allowed them to track and have a degree of access to D-Wave's hardware development and fostered amicable relationships with many inside D-Wave management. Before 1QBit was incorporated, and well before a formal partnership between the two companies was fully negotiated in 2013 , the founders were able to move their investment relationship to one that gave them a tentative if initially unofficial blessing to develop dedicated software projects. This understanding came with promises of equity-based D-Wave hardware access and training. Without non-paid hardware access any attempt at software development would be pointless, and D-Wave estimated the value of merely granting limited computational time on one of their processors at over a million CAD annually. This remarkable degree of access to a fairly secretive company, gave a privileged view of D-Wave that showed it facing many hardware engineering challenges, and that simultaneously developing software applications was a secondary concern that lagged behind in their internal priorities. Understanding what software even means in an annealing system takes a degree of familiarity that would not be available to the general public or outside developers. Being aware that at this point in time D-Wave was likely to be supportive of external developers that in some cases might later compete with their proprietary technology, which they had filed over 100 patents to protect, 
might have otherwise been surprising. Indeed, the lobby in D-Wave's Burnaby office contains a wall of plaques showcasing their registered patents. 1QBit would need its own patents to survive alongside D-Wave. To create the intellectual content to make into property, it would need some intellectuals. So it went to grad school.

\subsection{Mitacs}

The skillsets required to attempt programming the D-Wave from the outside are not easily assembled. It requires much of the same knowledge as D-Wave researchers but without the same access to hardware and results. In addition, it depends on an understanding of optimization practices and ways to translate mathematical problems into new forms while maintaining essential problem structures. For the small group of 1QBits' founders, finding and funding this labour was made possible by creating a flow of graduate students from a range of universities and disciplines into the structure of a company. A key technique to enable this flow to happen, was to develop relationships with Mitacs, and use its programs to access young academic researchers. Mitacs is a government funded organization that runs a series of programs aimed at placing current university students and post-doctoral fellows in an industry setting. These placements are described as "internships". However, they are unlike the typical role of an internship at most companies in Canada. These internships are aimed at creating new, novel projects that directly benefit the company hosting the intern as well as the student. Typically in a Mitacs project, a larger company would take on a handful of intern graduate students so that it could work on a 
collaboration with a professor or department at a university. However, the programs were able to be used quite elastically, and from the outset 'interns' outnumbered any other group at $1 \mathrm{QBit}$.

This scenario, enabled by Mitacs, made $1 \mathrm{QBit}$ able to conduct substantial amounts of research in its start-up phase in two ways. First, it provided a large subsidy of the payment each academic researcher would receive for participating. Often this constituted half or more of funding, which could rise as high as $\$ 65 k$ annually per intern. Second, it enabled access to otherwise unavailable labour sources. The majority of graduate students are under restrictions for how many hours per week they are able to work for compensation while enrolled in their programs.$^{66}$ This placement program provided a ready avenue to sidestep such restrictions. In addition, the majority of researchers 1QBit wanted to 'hire' as interns were not Canadian Citizens or (yet) permanent residents. This aspect of $1 \mathrm{QBit}$ 's demographics will be returned to below, but it is important to note here that foreign students are usually placed under strict workpermits that greatly narrow the amount and kinds of compensation they can receive. Mitacs also has its own network of Business Developers in many academic departments who canvas for interns on behalf of companies. In practice however, a majority of interns were found directly by the company, or approached 1QBit themselves, and were only later processed through Mitacs' channels. With this dynamic, Mitacs was transformed from being a organization

\footnotetext{
${ }^{66}$ This was furthered by a somewhat grey practice of allowing students who stayed with the company for multiple terms to qualify to receive shareholding options in addition to their funding.
} 
for connecting academics and industry, into a funding mechanism. The line between intern and employee in such an arrangement is blurry. By the legal/taxation standards of the company and also by university administration standards, these researchers were not employees and were often reminded by Mitacs program coordinators not to list themselves as such on their resumes or Linkedln. ${ }^{67}$ Yet, it is an understatement to say they conducted work that could reasonably be assumed to be done by employees of the company. After 1QBit had operated for two years and grown substantially, its reliance on Mitacs began to decline and be replaced by a more typical structure of funding industry research that relies more on programs like $S R \& E D^{68}$ for subsidizing technology development through tax savings. This shift obscures that initial flow of academic resources to commercial projects, without which it is uncertain whether the company could have accessed the talent it needed to produce the content discussed throughout this dissertation.

As previous chapters have noted, adiabatic quantum computing requires a range of expertise, beyond that of even the already established field of "quantum computing" that had existed in Physics' departments for several decades. Few faculty-level researchers in Canada focus on adiabatic-based computing, whereas alternative methods of quantum computing are more common, such as

\footnotetext{
${ }^{67}$ Instead, Mitacs relentless pursued students to get them to publicize their identity as Mitacs interns on social media. In many cases making it sound like this was a requirement of being in the program, even though it was not.

${ }^{68}$ Scientific Research and Experimental Development Tax Incentive Program http://www.craarc.gc.ca/txcrdt/sred-rsde/menu-eng.html
} 
at the Perimeter Institute. By assembling graduate researchers to focus on a research interest dictated by the company, Mitacs was a means for aligning a selection of academic and commercial research into a coherent vision of computing. The technology these students would be asked to innovate with was not deeply understood, had a low degree of functionality, and was not based on well-known computing theory. Even for those few researchers who deeply understood the annealing theory behind this computation, there were no known theories/methodologies of how to exploit such a system to solve a practical problem. Various expertise would likely be needed, but in what proportions was completely unknown. However, the lack of a clear underlying theory to base research was less of a problem in an industrial setting. Combined with their limited access to machine time and D-Wave personnel, this helped to conceptually blackbox the device. Instead, moving 1QBit's team away from the many questions about D-Wave innerworkings, toward the questions of how its inputs and outputs could be translated to match the better known forms encountered in academic and industrial literature and practice. As 1QBit grew it went from having 6 Mitacs students to nearly 30 at a time. The often changing proportionality of what academic departments these placements came from, reflected 1QBit's early shift from trying to understand a theory about quantum computing, to a practice of computing that requires a new interdisciplinary mindset. This mindset focuses more on the agencies and translation of the quantum actors already established in the network/machine rather than a deep understanding of their being or construction. For the company, this tended to 
mean a shift away from pure-physics applicants, in contrast to those being sought by the Perimeter Institute, and a greater interest in those with backgrounds in optimization, engineering, mathematics, and eventually software engineering. Occasionally researchers with connections to the Perimeter Institute applied to work with $1 \mathrm{QBit}$, which slowly became well known in many academic departments as a potential non-academic employer due to Mitacs. Usually these were turned away despite being experts in the field of quantum computing, as they were not expected to share the same vision for quantum computing being developed and propagated at 1QBit. As a mindset was developed by establishing an agreed upon set of relations between what was deemed technological, socially, and organizationally important, it began to act to refine and maintain the network.

1QBit's founders relied upon this combination of their unusual access to DWave's resources, an unorthodox academic-based workforce, and an uncertainty around what a quantum computing industry would be by its own stakeholders. The uncertainty around whether it was too early for value to be derived from pursuing specific applications created a space for $1 \mathrm{QBit}$ to insert itself. These pre-existing connections, unusual opportunities, and state of uncertainty by larger organizational actors, allowed $1 \mathrm{QBit}$ to put itself together out of the available business, academic, and technical resources. The company could initiate and accelerate their research and development without a clear theoretical understanding of the technology or a definite business plan that fit them into the 
existing computer industry. These plans/perspectives will be described later in the chapter as the promotion of a "quantum-ready" mindset.

\subsection{Quantum(?) IP and Corporate Positioning}

At the beginning of 1 QBit's research in 2013 , the quantum behaviour of $D$ Wave's qubits was receiving its most skeptical criticism from the academic world (Aaronson 2013, Troyer 2013, Shin et al. 2014). While the D-Wave could be shown to be working, in that it solved mathematical problems, the initial skepticism over whether its functionality came from quantum effects, or for example classical magnetic effects, created pressure to insulate 1QBit as much as possible from criticism directed at D-Wave, and more importantly to not be overly technological dependent on D-Wave as a platform. This pressure was increased by broad speculation that Google's recurring interest in the technology would lead them to develop their own hardware along similar lines. ${ }^{69}$ Though separate and somewhat contradictory, the concern that D-Wave may not in fact be quantum was compounded with the prospect of multiple platforms for adiabatic quantum annealing technology, to encourage $1 \mathrm{QBit}$ to find ways to frame the relationship of $1 \mathrm{QBit}$ 's software development to hardware manufacturers in somewhat ambiguous terms. This expressed itself in many small ways. For example, a common pattern of sidelining academic criticism to potential industry partners/clients by claiming that $1 \mathrm{QBit}$ "did not care" if D-Wave

\footnotetext{
${ }^{69}$ Google's Quantum Lab currently works with D-Wave hardware, but has shown interest in designing the next generation of quantum annealers.

https://research.googleblog.com/2015/12/when-can-quantum-annealing-win.html
} 
was quantum "as long as it's faster"; stating in one case "we are not interested in debates about quantum states, we care about solving hard problems faster". Yet, at the same time, prominently pushing "quantum" in their name, website, and other self-descriptions. Just as often, 1QBit would defend the quantum-nature of the qubits they were programming, especially as other academic research supported them. Rather than running over the many kinds of internal and external statements $1 \mathrm{QBit}$ made to navigate their tricky, if usually amicable, relationship to D-Wave, I focus here on a key translation where these dynamics collided: the writing of patents.

Patents confer legal protections for an invention, particularly the ability to exclude others from using or selling it. However, to apply and receive this protection, the invention must be explained in detail, made public through patent registration databases, and meet various legal criteria relating to the originality and type of process being protected. In Canada, patents expire after 20 years. The trade of increasing public knowledge in exchange for being granted a limited time of exclusory rights is frequently more complicated in practice. Patents often reflect the tensions in many companies that want to protect their intellectual property. The description of a technical object for protection can be constructed in many forms. The form they do take reflects a company's overall strategy (Bowker 1994, Graham 2012). This can include sneaky tactics, such as filing fake patents to clutter the public record of a company's activities or protecting technologies to keep them from competitors. As Bowker (1994) notes, a patent simultaneously "reveals and obscures". Patents bring a process into the public record in order to 
gain legal protections over specific technologies. Yet, companies simultaneously try to make patents generalized and unrevealing of daily corporate activities, attempting to retain some level of trade secrecy.

As a small company, 1QBit required patents to protect their innovations and to raise the valuation of the company to outsiders. Patents, just as much as software, act as the product of $1 \mathrm{QBit}$. Without patents it is unlikely that a company would buy $1 \mathrm{QBit}$ or that it would receive significant investments for the projects mentioned in this dissertation. Patents are such an important piece of content for the company that after assembling its first research team in May, 2013, 1QBit had begun the patenting process by June, 2013. Given the nontrivial cost of patenting for a startup,$^{70}$ this indicates the importance of patents in emerging computational technologies. Patents in this sense protect not just an intellectual property, but positions in the layout of an industry. Since the essence of 1 QBit's technologies are methods of translating problems into forms amenable to quantum computing, this attempts to put them in a mediating position or "passage point" (Callon 1986), which other actors must first interact with and submit to in order to access quantum technology, as well as a drive for them to recruit others into quantum-based computation. Patents are required to create and secure this passage point. What the company aims to do with a patent, is not just protect the work that has been done, but also future variations of that work and attempts to circumvent their protection. The patent in this way reveals an

\footnotetext{
${ }^{70}$ Including the time paid to a lawyer to thoroughly review a patent, the filing of a single patent can easily reach $\$ 30,000$.
} 
expectation of the space of possibilities the actors it describes will occupy, and how others may conceive of them. Patent strategy has been a long held area of interest when examining socio-technical systems and their corporate environments (see: Hughes 1983, Rech 1985, Bowker 1994, Arns 1997, Graham 2012). Patents are often highly technical documents, but written in a specialized legal form, and deployed for many social/business reasons, including the establishment of credibility.

Prior to $1 \mathrm{QBit}$, several patents related to quantum software and quantum computers as a whole can be found in the Canadian and American Patent Office Databases. With some exceptions such as those filed by D-Wave, these reflect the limitations of patent protection and the risk of describing a patent in too general of terms. Merely being granted a patent is not enough to realistically claim domain over a process or invention. For example, the registry of preexisting "quantum computer" patents ${ }^{71}$ did little to impede others when the vague claims over that technological area failed as a business strategy because they did not hold up to the expected standards of the test of a courtroom. Patent databases are full of useless patents, perhaps best illustrated by the "Colouring Book of Patents" released by the National Archives of the United States (2016). A patent's authority comes from its perception to withstand a hypothetical courtroom test, or from having actually been litigated. These require an agreement that the patent is a faithful translation of the real-life activities it claims

\footnotetext{
${ }^{71}$ For an example see: PATENT \# US6472681 B1
} 
to protect. With this constraint in mind, 1QBit needed to find ways to describe their IP in the broadest possible terms. This required an ongoing conceptual and terminological negotiation between a researcher, a manager, and a patent lawyer on each project for which they sought a patent.

While many patent lawyers specialize in patenting scientific research and development, it would be unrealistic to find one who could judge at face-value the patentability of a technique developed for such an exotic computation system. For example, in the process of developing one patent for an Integer Optimization Toolbox (IOT), eight 'tutoring sessions' for the patent lawyer were held over a four week period. These were in the form of conference calls that served to explain the initial description of the method written by the researcher and allow the lawyer to pose various questions about the context and workings of the invention. Listening to the calls revealed a process of teaching researchers about patent laws, and patent writing conventions, as much as lawyers about quantum computing. Through these back and forths the technological description was iterated toward the genre of patents, while a legal-logic was simultaneously spread to researchers. ${ }^{72}$ Meanwhile, the draft patent would be passed back and forth numerous times, slowly morphing from white paper to filable patent.

\footnotetext{
${ }^{72}$ This logic became slowly institutionalized, as researchers shared their experiences with one another in crafting patents, and some researchers with an affinity for the genre were frequently consulted. This was encouraged in part because of the costs of legal fees, which created incentive to pre-legalese patents as much as possible to minimize the work done by lawyers.
} 


\subsection{Papers to Patents}

A helpful example of the careful negotiations around these document iterations can be seen around the presence and classification of the machine, and by extension the presence or absence of qubits. Prior to patenting, researchers in most cases at 1QBit had written white papers to explain to their peers the innovation they were working on. These papers were written in an academic style, mimicking the layout, typography, and writing conventions of academic journals. The language of these would typically include a combination of the author's academic discipline and the mixed terminology local to 1QBit. After a patent was secured, these papers were sometimes circulated externally to various research partners and were designed to reflect the rigorous and scientific image that $1 \mathrm{QBit}$ wanted to be able to draw upon in marketing itself to other corporations. These papers frequently referred to "D-Wave"; in this case meaning the 512-qubit quantum annealing processor Vesuvius, and the innovations they were describing were listed explicitly as for D-Wave platforms. This is, after all, the machine they were actually working with and thinking about day to day. In almost all cases at the time, this particular model of processor was used, including for the IOT. Though eventually a more advanced version of the computer was produced, it was not as readily available. Under advice from the lawyer and 1QBit management to reduce the dependence of their descriptions on another company's product, alternative ways of describing their innovations would have to be found. 
As the researchers attempted to move further away from a discourse which explicitly invoked the D-Wave in its patents, they quickly ran into the problem of describing its techniques instead in mathematical terms. This was partly due to the situation that the researchers who worked directly with the patent lawyer were in most cases mathematicians, and when directed to avoid referring to specific machines, naturally abstracted to the mathematics that made their innovations work. However, a particular mathematical function cannot be patented. Neither can a "scientific principle or abstract theorem". ${ }^{73}$ For example, an initial attempt to define a method in quantum annealing to search energy landscapes, that described the relationship as a grid of hypercubes ${ }^{74}$ where "each hypercube represents an affine transformation of the original QUBO," quickly raised this concern for its strictly mathematical language and relationships. In order to ground this process as a patentable method, mathematical objects like hypercubes and QUBOs needed to be put into relationships with material machines that are inseparable from the process. This required bringing the $D$-Wave back into the description while at the same time obscuring it.

Several drafts of each patent moved through different terms for characterizing the role of a D-Wave computer. Each of these served to recontextualize the role of the D-Wave machine, and of D-Wave the company, as not inherently required

\footnotetext{
73 http://laws-lois.justice.gc.ca/eng/acts/P-4/index.html

${ }^{74}$ Hypercubes are fascinating higher-dimensional objects. Numberphile provides a fun and insightful introduction to the concept for the layperson: https://www.youtube.com/watch?v=2s4TqVAbfz4
} 
for the invention. Early drafts of the IOT patent used "D-Wave", then shifted first to the slightly more general "adiabatic quantum computer" and "quantum annealer", before moving to the much more general "analog computer". Each term change was part of a new draft that circulated and debated for how its connotations suggested the reader conceptualize the technology/innovation. All of these terms are valid descriptions of devices such as Vesuvius, but imply a very different category of technical system, with historically different areas of application and relevance. Calling the quantum-based device a computer suggests certain requirements such as programmability, which could be argued an annealer does not have in the same sense. The shift to "analog computer" alters this expectation of general programmability and could mean a host of systems very different from a quantum computer as well as from a digital computer. It became clear through conversations between the mathematicians and lawyer that the truly novel work to be protected was not actually the quantum aspects of the system at all, but instead the preparation and translation of problems into a form that was now valuable given the existence of the new processors. Rather than quantum computation, the method for creating and solving QUBOS with machines that can meet certain optimization criteria became the focus. This still requires a description that is more grounded in material technology and is less mathematical. However, it creates a way to describe a technical system, and method for using it, that makes the truth about the quantum nature of any machine irrelevant, placing the company as a more neutral developer. 
This thinking shifted the description of the method in two ways; first, from using a "D-Wave processor" to using instead a "Turing Machine with access to an oracle". An "oracle" is a mathematical term that describes any computation device that is not a Turing Machine, and provides an answer to a problem in a single operation. In the case of D-Wave, a single operation would be an anneal. In the IOT patent, a D-Wave would still be listed as an example of one such an oracle, but not the definition of one. Second, on the insistence of the patent lawyer, the technical description of the role of digital computers increases, which is initially a confusing request in the minds of the researchers. This includes frequent references to example instances of using the method - down to references of example monitors, mice, and desktop computers. The inclusion of such items serves to increase the variability of contexts in which the method could be used, eliminating others from sidestepping aspects of the innovation and claiming a new method.

Of primary interest here is not the particular word used to describe one of the machines or the many embodiments of the method and its system. ${ }^{75}$ That may change in the next patent, and in some patents written near the same time as IOT other generalizations were considered more advantageous (e.g. quadratic programming machine). What I want to emphasize here instead is the shift in perspective implied by these transitions in terms. They establish 1QBit's role in a

\footnotetext{
${ }^{75}$ It was, however, interesting to note the many descriptions of what constitutes a "user" of such a system includes both humans a variety of nonhuman machines and programs.
}

Noon 230 
new way that reflects their larger business strategy. That strategy is codeveloped with legal strategies, in response to often unexpected innovations, and the individual knowledge and terminologies of researchers. The change in term is not simply a result of a manager telling a researcher to find a new word, or of improving the accuracy of a description, but of the researcher and lawyer sharing the concern and slowly seeing different ways to reconstitute an innovation. When describing the difficulty in defining ideas for this process compared to in academia, a mathematician described their experience in shifting to this mode of description:

There's this new part I'm learning how to do [...] coming up with something that can be protected as a patent. That is very interesting! That part is really hard [...]. Here we have to protect it, and we have to protect it in a way that the method has potential of solving lots of different things, and the way you would use it to solve those things are not the same. You actually have to change them. But you want to predict something that has to be done in all of them. So you come up with a complete example [...] a complete intersection for what has to be in all of them. If you wanted to write that method as one algorithm then what would that algorithm be?

Finding the "complete example" is an activity researchers find extremely different than the theorization or results produced for academic writing. This new goal requires them, to an extent, to learn to think like lawyers and managers without leaving the terms of their expertise. The struggle to find a "complete intersection" 
reflects the shift in concern created by putting the mathematician into a new legal-corporate set or relations, where not only a new local scientific-legal language must be developed, but a view of innovation and technology. This shared view is required to create the legal description that takes into account the business strategy of management while retaining a faithful translation through the path of the socio-technical actants that make up an innovation, to the white paper, and finally to the patent.

Software programming technology, such as the example described in the previous chapter, shows how the local interests of particular actors and the characteristics of a problematic were erased after the fact to create more generalized functions as well as descriptions of those functions. The many failures along the way in that example were replaced with a success constructed from the conditions created by those failures. Unlike some companies that work to maintain maximum secrecy around their patented materials, despite the inherent publicness the patent process is meant to require, 1QBit is in the position of needing to promote the details of its technological innovations. With a patent in place it can engage other organizations at a more granular level, and attempt to identify itself as a more credible organization both in technical knowhow and business value. Patents themselves are not typically read by many people, but they allow circulations of other kinds of texts. They are a communications strategy as much as a text themselves, creating new potential channels of discourse, and relationship building. In order to produce this mobility, the patent writing process had to first tackle and align a series of concerns. The 
lawyer enters the conversation with a set of criteria for the patent. To meet the hypothetical courtroom test and the immediate scrutiny of the patent office it must meet certain criteria (i.e. being new, useful, non-obvious, etc.). Since what is being sought is the protection of a method, rather than copyright over software, the lawyer aimed to tie the innovation to certain material operations and devices. Management, on the other hand, needs the protection to be as broad as possible, and to make it valuable to as many investors as possible. For the researchers, they need to make everyone else understand what innovations they have created. In aligning these views each human is standing in for, describing, and defending their respective nonhuman: the courtroom, the company, and the qubit. ${ }^{76}$ With patent in hand and white papers mobilized, new relationships are potentially opened up. 1QBit's network can now enter into the larger network of the quantum computing industry and begin to attempt to claim itself a passage point.

\subsection{Hocking Applications-Driven Development for Credibility and Access}

There are a small handful of research teams that could be characterized to differing extents as "developing software" for quantum annealers. Some of these teams also predate 1QBit. These include groups at Lockheed Martin, Google, DWave, NASA-Ames and the USRA ${ }^{77}$ Research Institute for Advanced Computer Science (RIACS). The majority of these teams were initially assembled in 2011

\footnotetext{
${ }^{76}$ For readability I say qubit, but a fuller description of the nonhuman behind the scenes here is the network of connections around the qubit the make up the qubit's programming. These involve many material actants as well the theories and variables they have translated.

${ }^{77}$ Universities Space Research Association. When fully acronymed: USRA-RIACS.
} 
and formed to work on hardware implementation challenges, acting as testers to validate the technology for their respective organizations. For example, this would typically mean having a concentration of expertise around the physical interactions of quantum annealing, rather than the implementation of constraints in an optimization problem. Each of these organizations did not purchase their own $\$ 15$ million machine to work with, though it is often presented this way in news coverage of their activities (Grossman 2014). Google, NASA and USRA work on the same computer, which was acquired in a joint public-private research project known as a "Space Act Agreement". ${ }^{78}$ This shared machine is housed at the NASA Advanced Supercomputing Center (NAS) at the NASAAmes facilities. However, these teams work independently ${ }^{79}$ each allotted a share of available computing time ${ }^{80}$ Google has, in several stages, released public reports on the kinds of problems it runs on this machine. Its announcement in December, $2015,{ }^{81}$ for example, noted results that showed a $10^{8}$ times speedup compared to a digital processor, on what it described as "proof-ofprinciple" problems (Boxio et al. 2015). These problems are extremely abstract computations tailored to the processors design, but as the Google team noted in the same announcement, of essentially no practical application.

\footnotetext{
${ }^{78}$ For more on the mechanisms and limitations of partnerships via a Space Act Agreement, see: https://www.nssc.nasa.gov/saa

${ }^{79}$ In some cases, staff have also been hired by Google who were originally working on the NASA team.

${ }^{80} \mathrm{http}: / /$ www.usra.edu/quantum/

${ }^{81} \mathrm{http} / / / g o o g l e r e s e a r c h . b l o g s p o t . c a / 2015 / 12 /$ when-can-quantum-annealing-win.html
} 
Unlike 1QBit, these teams are less inherently invested in the immediate broadening of the quantum computing network, though the growth of the industry does benefit them in different ways. Yet, the majority of their work is not catalyzed around particular problems, but general testing and benchmarking of the quantum processors. These groups had been persuaded by D-Wave's prototype pre-commercial models, made up of 128 qubits or fewer, that this approach to computation could become another hardware resource that can be integrated with their other supercomputing architectures. Their teams have since been focused on finding ways to optimize the use of the hardware rather than finding ways to apply it to real-world problems. Each of these groups initially relied upon D-Wave for tutelage, installation, and technical support, in a vendormodel that harkens back to IBM selling and support of the 1950s-1970s (Hill, Jouppi and Sohi 2000). Lockheed, for example, publicly stated in 2012 that they believed the D-Wave could be used to perform bug-checks (i.e. software verification and validation) on the complex systems used in its fighter jets (Novet 2013). However, as they worked with D-Wave to refine software for this task, it became clear that such a large-scale real problem involved too many variables for D-Wave's current processors and could not be approached. Unlike the example in the previous chapter of continuing to translate a problem and its solution, organizations in this position have assumed that more basic research is needed separate from and as a prerequisite to their particular applications. Most immediate expectations of creating practical applications were shelved in favour or contributing research and computing time to more low-level engineering issues 
until there were thousands of qubits available to program. ${ }^{82}$ This poses two views of the progress of quantum computing technology. The first, suggests quantum computing as a component in a constantly updating and rearranging hybrid system, and the second of a pure quantum technology that must scale and perfect its inner workings before becoming relevant.

NASA-Ames faced a similar dilemma as corporations like Lockheed whose industry problems seemed ill-suited for the current quantum processor. The form of optimization problem annealers can solve is theoretical relevant to a great deal of tasks the agency performs, such as complicated scheduling, packing, or energy and resource allocations. The annealers were not expected to be realistically even tested on these problems for several generations of hardware development. However, the significant price tag for the machine and the academic controversy around it, put pressures on the NASA team to demonstrate that it was a worthwhile investment, or at the very least, that it actually worked. NASA had not only contributed to purchasing a machine, but had hired a team and set up a lab dedicated to working with it at NASA-Ames.

The NASA-Ames' team took a basic-research approach that did not produce results that easily showed the potential value of the technology to the larger organization of NASA. For example, they worked on better understanding the physics at work in the machine, the strength of individual connections between

\footnotetext{
${ }^{82}$ Currently, these groups work with machines that have between 1000-1024 programmable qubits.
} 
connected chains of qubits, instead of being geared toward an applicationsfocused project. While this approach makes sense as a way to fundamentally validate an emerging technology, it also put pressure on the NASA-Ames team as a publicly funded agency, to justify its research to the larger organization of NASA at a time when the technology's developer faced frequent criticism from the academic community over its controversial approach and marketing. It is, however, not surprising that NASA had taken a chance on developing quantum annealing computation. NASA has long played an important role in the transfer of new technology both between the public and private sector, and between those sectors and other US government agencies, including the NSA and CIA (Bozeman 2000, Span, Adams and Souder 2002, Hersch 2016).

Creating a point of contact between a small startup in Vancouver, BC and a NASA laboratory in Mountain View, California, required a number of factors. 1QBit's applications focus may have been seen as appealing to the more established users of D-Wave computers, or just as likely seemed naive. But they had little reason to take a year old startup seriously, let alone give them access to their accumulated knowledge. For $1 \mathrm{QBit}$, such contact provided an opportunity to grow their technical knowledge, and even to elevate the prominence of their research, positioning themselves as the avenue for specific outside problems to become relevant to the industry.

In order for 1QBit to speak for quantum computing outside of this relatively small user base, these established and well recognized actors needed to be put into a 
clear relationship with them. With their work patented, 1QBit was better able to mobilize its technology as part of meetings and introductions to persuade the current users to give them access to their communities. With its public status and non-commercial priorities, NASA was the clearest 'point of entry' into the knowledge base of those who operated their own D-Wave machines. NASA was the least likely to be directly competitive as a software developer, and saw its output as being publishing papers rather than patents. Being able to connect with NASA would require a series of coincidences, resources, and opportunities.

\subsection{Connecting Coincidences}

Initially, 1QBit needed to prove its relevance and technical competence to NASAAmes. Unrelated to quantum computing, two of $1 \mathrm{QBits}$ researchers had briefly studied an optimization problem called cognitive radio in an engineering course at UBC. Cognitive radio involves the "intelligent" or dynamic use of a wireless communication that effectively allows more users to share a selection of bandwidth. Cognitive radio finds ways to arrange many transmissions from users from a limited number of base stations (i.e. radio towers, cellphones, satellites) at the same time. There are many classical strategies employed to best optimize these transmissions, as it is a non-trivial NP-Hard problem. When told of NASA's desire to find a practical application for their annealer, these researchers investigated the mathematical structure of cognitive radio further. Somewhat surprisingly, it ended up being a problem that could be translated to a QUBO relatively easily even for a realistic size of the problem, and therefore run competitively on the current quantum processor. Finding a problem that was this 
much of a good fit is extremely rare. This project was at first brought to the attention of another laboratory at NASA, the Jet Propulsion Lab (JPL). After an informal meeting between a 1QBit researcher, two executives, and JPL staff, JPL was given 1 QBit white papers that they could circulate within NASA more broadly. This interest helped create awareness of 1QBit as working in application areas relevant to NASA. With the interest from JPL and recommendation from DWave, 1QBit had enough convincing organization-allies to gain some access to NASA-Ames and its partnered users.

The NASA-Ames team ${ }^{83}$ and 1 QBit shared an overlap beyond their access to DWave that increased the odds that they would attempt to work together. Some of 1QBit's founders had attended the educational program/accelerator known as Singularity University (SU). This semi-academic program runs at the NASA Research Park, sharing a campus with NASA Ames and promotes itself as having strong ties to the silicon valley investor and start-up communities. ${ }^{84}$ Members of what would become the NASA-Ames' research team had also attended SU's graduate program. SU espouses a strong techno-utopic rhetoric, and its programs encourage the use of cutting edge technologies to directly address social problems and "transform the world" as the SU website and

\footnotetext{
${ }^{83}$ While for simplicity sake I refer to NASA-Ames, which reflects a larger umbrella in NASA but also a specific location, the particular section of NASA being referred to is the NASA AMES Research Center's Quantum Artificial Intelligence Laboratory (NASA-AMES QuAIL).

${ }^{84} \mathrm{http}: / /$ singularityu.org/
} 
program descriptions frequently state. ${ }^{85}$ The accelerator program itself connects students with business and technical backgrounds together to work on commercializable pilot projects, and focuses heavily on connecting venture capital to research projects. $1 \mathrm{QBit}$ maintained close ties with $\mathrm{SU}$, regularly speaking at SU conferences where they functioned as an example of the futureis-now disruptive tech that SU promotes as revolutionary. 1QBit's first official board member was an SU faculty member and vice-president. ${ }^{86}$ This previous connection facilitated members of $1 \mathrm{QBit}$ to approach NASA researchers casually and propose various working arrangements before establishing any official relationship. With these research and interpersonal connections in place, 1QBit could get its figurative foot in the quantum computing door.

On September 10, 2014, NASA-Ames held the first "D-Wave users Colloquium". 1QBit was invited to present alongside presentations from NASA, Google, DWave, the University of Southern California's Information Science Institute (ISI) and Lockheed. The colloquium was to explore how these research teams could work together or share information, with a focus on connecting NASA and 1QBit researchers who gave the majority of presentations. After a flurry of NonDisclosure Agreements, nine members of $1 \mathrm{QBit}$ flew from Vancouver to Mountain View, California, to present their work to the NASA team and other

\footnotetext{
${ }^{85}$ SU programs focus on "disruptive" and "exponential technologies"; it describes technology as prone to rapid shifts that determine changes in economic and social life.

${ }^{86}$ David Roberts bio reflects the web of interests represented at SU, with connections to silicon valley and the US intelligence community.

http://experts.singularityu.org/speaker/a0pF0000005djIEIAQ
} 
guest research groups. Setting the tone of the colloquium, one NASA host began by noting, "It's Important we trust each other and at the same time maintain discretion". Information was being shared, but it wasn't free to roam.

At this event, 1QBit presented its cognitive radio project and a "graph similarity" application. Graph similarity was a project led by a postdoctorate at $1 \mathrm{QBit}$ which was developed to compare how similar a pair or set of molecules were to one another. Representing molecules as graphs allows for comparisons useful in cancer detection and drug discovery. While these applications were not directly of interest to the groups attending, demonstrating the ability to practically solve computationally difficult graph matching problems has a much wider applicability. For example, Google conducts extensive work using graph theory in its data analytics and services (Monea 2016). Such overlapping areas of interest created conversations that demonstrated how $1 \mathrm{QBit}$ had overlapping matters of concern with other D-Wave users even if they were not focused on the finance applications 1QBit promotes. It also depicted them as the most applications focused team working on quantum annealers, and to their surprise, one of the larger teams.

Even limited information sharing with NASA does not come easily. After traveling $1500 \mathrm{~km}$ and staying at the NASA Exchange Lodge at the NASA Research Park, the $1 \mathrm{QBit}$ team did not go the final $1 \mathrm{~km}$ to meet where NASA-Ames actually conducts their research. Instead, the group used conference space at the SETI Institute and was formally hosted by USRA. This shift in venue was in part the 
result of concerns over the nationality of several members of 1QBit's team and the likely denial of access to the restricted areas of NASA-Ames that is part of a military base and where their quantum computing lab resides. Out of 1QBit's original six person research team composed of students from the University of British Columbia and Simon Fraser University, four were Iranian citizens. As the team grew to over twenty, many other nationalities were represented, including Chinese, Egyptian, Israeli, Indian, Chilean, and even a few Canadians. However, this notably high representation of Iranians in a Canadian company that was populating its research teams from $\mathrm{BC}$ graduate students, was a trend that continued as $1 \mathrm{QBit}$ expanded. Rather than being coincidental, especially in the course of developing the company's foundational technology and models, this is influenced by the Iranian educational system. The international demographics of 1QBit's researchers reflects the local decisions of different mathematics curricula and the somewhat arbitrary placement of some areas of mathematics as central or advanced in those systems. Interviewed researchers consistently noted the preferences of different local mathematics.

One researcher noted that in mathematical journals "they talk about Chinese mathematics $[\ldots]$ there is a Persian mathematics $[\ldots]$ it depends on the education system. What is basic mathematics, what do you want to teach the kids. What do they teach in the first year of university, and stuff like that". Discrete mathematics is taught in high schools in Iran and is more familiar to students entering undergraduate programs and influences what areas they are likely to be pursued at higher levels. This meant while Iran taught discrete mathematics, there was 
very little calculus. "Whereas a Chinese education system does more number theory [...] in North America they do much more analysis, they do very little discrete mathematics, they do more calculus. That's why the culture of mathematics [in Iran] is more discrete, and we have lots of Persian researchers [at 1QBit] so they are more comfortable with discrete research".

In addition, researchers from Iran felt that the Iranian graduate school system was underdeveloped compared to its stronger undergraduate system, leaving students who wished to do more advanced and original research to look to an international education. This perception was remarkably consistently held by researchers at 1QBit who had attended graduate schools in both Canada and the Middle East. ${ }^{87}$ This diasporic approach to graduate education created concentrations of Iranian students in some Canadian STEM universities who were well versed in the type of mathematics ${ }^{88}$ (i.e. combinatorial analysis) used to describe quantum annealing systems. In addition, this area of mathematics is less likely to be as intuitively understood by new graduate students who went through the Canadian educational system. Exactly why each country selects certain kinds of math as either more valuable or more advanced in their educational systems is beyond the scope of this dissertation (see: Schoenfeld 2004, Schmidt, Wang and McKnight 2005, Appelbaum et al. 2009). However, the outcome creates, if not monopolies of knowledge, concentrations of expertise

\footnotetext{
${ }^{87}$ This consensus has an obvious bias, however, as those who felt the Iranian system was as strong in graduate work be less likely to be educated abroad.

${ }^{88}$ It soon became a running joke among management upon hearing a resume had been submitted to ask if they were Iranian.
} 
and the research interests connected to that expertise. The development of this concentration of expertise in Vancouver provided 1QBit with a pool of expertise not as easily available to a US government agency, adding an unplanned value to 1 QBit's inclusion once such red-tape could be sidestepped with strange hosting arrangements.

The inclusion of $1 \mathrm{QBit}$ at this colloquium allowed the company to learn from the rare and important technical resources of the 'users' that made up the industry at that point. However, management at 1QBit saw the biggest gain as being the association and credibility derived from a more formal relationship with NASAAmes. The sharing of technical knowledge was just as much a tactic to develop stronger organizational ties that featured $1 \mathrm{QBit}$ in a particular role. This led to the publication of co-authored papers with NASA-Ames and 1QBit. It also allowed 1QBit to list NASA-Ames as a partner to potential clients, potential hires, and the general public, providing a "dressing up" of their work as equally scientific as it was commercial (Bowker 1994). As a non-corporate partner, NASA helped elevate 1QBit's profile as a legitimate producer of new scientific and technical knowledge, but only after it had produced a great deal of the scientific work that allowed it to establish this relationship.

\subsection{Quantum Ready}

Having gained access to the dominant actors in the field, and established a degree of credibility and leadership, 1QBit has had to evolve a mindset around quantum computing that justifies their approach. This mindset is simultaneously a 
result of the kinds of applications their research has produced and an argument for their potential importance in the industry. Part of their success has come out of the way their mindset has embraced the limitations of the technical actors in the network. In 2015, 1QBit formalized this mindset internally, branding their company and its software as being "quantum-ready". This was formally presented by management to everyone working at the company as how to conceptualize their identities, goals, and technologies. It included internal posters in the style of scaling benchmarks meant to communicate the blend of hybrid technology and anticipatory thinking this motto implies.

The results of quantum computing to date, the problems solved and their benchmarks, are not very impressive when compared to the most advanced digital alternatives. Several times quantum annealers have been reported as having massively faster computational times than digital computers, but in each case critics have pointed out that other digital computers (i.e. those running less generic digital-based algorithms) could still outcompete quantum annealers. Due to this, the most frequent measurement circulated by D-Wave and 1QBit has not been the benchmark of a computation time, but that of scaling. Scaling projects the abilities of present quantum annealers with those of future models, anticipating the quantum equivalent of a Moore's Law. The scaling metric emerged as an early part of D-Wave's model, appearing in charts presented by their CEO, CTO, and engineers as "Rose's Law", after D-Wave's co-founder Geordie Rose. Being a projection rather than a true measurement invariably 
creates inaccuracy and the rate of scaling has been adjusted several times as the reality of qubit numbers doubling has slightly slowed down. Qubits are fussy, and connecting more of them requires many difficult material accommodations that cannot always be met. Still, the scaling chart indicates a hypothetical point at which quantum annealing processing power overtakes digital computation. In a similar dynamic to peak oil, this point indicates a sudden turnover where a quantum prototype would become a fully functional and superior paradigm. To date, that point has remained about two years away, consistently, for the last four years. Whether it can be reached by 1 QBit and its partners is uncertain. Being quantum-ready acknowledges this uncertainty around the efficacy of current quantum technology, and creates a role for the company to not only build software that is flexible in the extent it draws upon quantum hardware, but also build itself around the widely accepted idea of a quantum computing transition in the midst of mediocre results. With the credibility of publications, patents, applications, and prestigious associations, $1 \mathrm{QBit}$ is able to spread its mindset on platforms like the World Economic Forum and community building websites such as "Quantum for Quants", ${ }^{89}$ which it uses to create conversations and interests in the future target finance markets of its problematics-software. In this role it evaluates clients' technology and problems to explain how and when they could be affected by the coming hypothetical peak-digital quantum scaling point. Shifting from software development to consultancy as a business strategy has a clear benefit of creating another stream of revenue for the company. In addition,

\footnotetext{
${ }^{89}$ http://www.quantumforquants.org/
} 
it shows how a corporate actor develops tactics to act as a point of passage in a shifting network.

The growing awareness of quantum computing generally, as seen in the reaction to Prime Minister Trudeau's press conference, has created an increased acceptance of such a quantum tipping point as an accepted reality that will drive development. Taking advantage of this to push forward the company's own version of quantum computing has meant spreading a coherent vision of how and where to do that computation across students, funding organizations, papers, patents, competitors and partners. Each of these require different techniques to access. The development of quantum-ready emerged out of the interactions with all this groups by a company that did not know what it would produce until each of these were incorporated into its network. The mindset did not guide these actors all along, but in classic ANT fashion, was produced by the process of their mutual entanglement. 


\section{Chapter: Conclusion: Computing in Uncertain Times}

The closer I tried to get to the glowing boxes, physically and in understanding them, the more the network of quantum computing with its many heterogeneous components came in between. Their current existence and implications could only be appreciated in relation and through the many people, representations, and contexts to which they become tied. This effect was reciprocal.

Understanding what quantum software engineers, 1QBit code, or CEOs were doing only made sense in relation to the fluctuating existence of qubits, machines, mindsets, and their negotiated agencies. These socio-technical relationships characterize the process of developing computation, which emerges here as an effect of linking together heterogeneous elements into a network. This network requires us to consider many new objects as part of the world of computing, and sidelines other familiar aspects of computers and their operations. Building this network requires its actors to revisit these fundamental assumptions as well, renegotiating the boundaries of what we think of as computer hardware, the work of software, and the way the expectations for both are institutionalized in organizational practices and visions of that network.

My account of a quantum computing network shows how well established divisions of computational work between hardware, software, and institutions are complicated by translations that require these conceptual containers to be left open and flexible. At the same time, the examples detailed in these chapters 
show the digital is not cleanly replaced, erased, or departed from. It remains an important context that helps locate and assist the development of quantum computing. At times the digital provides an important reference point used to define the quantum against, and a logic/set of technologies that hybridize with quantum materials and paradigms to produce complex, potentially transitional, computation systems.

Qubits are one of many technical actants required to make the computational network examined in this dissertation function. They are certainly the most novel, symbolic, and influential for fostering a new paradigm. The creation of a quantum bit is seen as the arrival of a new disruptive actant. However, it does not arrive on the scene abruptly. Qubits were not discovered. Through many social and technical relations qubits become defined, and occasionally redefined. The conditions that must be negotiated for them to identify as qubits are multiple, requiring a carefully constructed environment where materials are technologized and connected to different social domains. The qubit is conceived in many ways, each expanding its diversity of social functions, from corporate promotion to programming. Much of the uncertainty about the future trajectory of this computational network can be traced to the material qualities of the qubit's embodiment, and the broadening range of agencies other actors attempt to distribute across their unusual materials and structures. While in many ways the qubit serves as the foundation of a new paradigm, its conceptual flexibility, rather than solidity, helps the network grow into new areas. The more ways its definition 
can be recast, the more it can do. In the corporate lab, this is most commonly posed as a question around scaling a technical architecture while preserving certain computational characteristics. However, a socio-technical view of this poses the challenge as a broader ontological process, where reality must be generated/constructed by building out many relationalities through a multitude of translation techniques. This is a process not well depicted by the category of 'hardware', which tends to be the default approach to explaining what quantum computing is. It is also left out of disruption narratives of technological change, which miss out on the relations and hybridization of emerging technology with established socio-technical regimes.

The evolution of a hybrid software framework for designing, implementing, and flexibly iterating quantum computing programs, reveals the central role of problematics in computation. Software design and coding often mean dealing with problems: bugs, delays, unexpected results, or user interface issues. However, the struggle to create software in a new computational network highlights how an underlying method of solving problems is native to computational architectures and must be translated into problems deemed useful to solve. Establishing problematics and translation methods requires finding contexts to translate them into, which can provide criteria and stepping stones to develop the technological network. The "heterogeneous engineering" (Callon 1987) this entails is considered to an extent by socio-technical approaches to programming (Ende and Kemp 1999, Sommerville 2011), but with a broader set 
of organizational interests, outside actors, and material agencies. This is particularly influential in non-general computing structures, but also brings out the assumptions of the differing problematic in digital systems. To get to the stage of design/troubleshooting commonly associated with coding software applications, requires developing a conceptual problematic that faithfully translates the range of technical actants available to program. The technical and social legacy of digital computing created many resources that the quantum network can draw upon: fabrication techniques, coding resources, etc. Just as useful, the digital provides a set of limitations and choices that the quantum can define itself against. This can particularly be seen in the search for new problematics for intractable problems - made so by the expectations and developments of the digital. The long, unchallenged success of the digital is what makes any quantum computing technology so noteworthy. Developing and deploying this new computing network requires a new kind of competency that can link qubit's representations/problematics to other matters of concern. The production of quantum software invariably requires and co-produces quantum software engineers and companies. Rather than replacing a digital system with a fullyfunctional quantum programming paradigm, the novel characteristics of quantum annealers are bridged/hybridized with digital systems. This hybridizing process creates a new hybrid system, and allows the introduction of technical components and their consideration into broader social/business settings. This reveals the shaping power of specific domains to the early spread of new computational networks, though does not suggest that these domains are 
determinative. Rather, they are vehicles for network growth and finding ways to make quantum computing meaningful.

When quantum computing was theorized as part of a subset of ideas in quantum mechanics, it had a relatively well defined place in a small network. This network was made up of physicists, physics courses, select journals, and similar components. During this stage, it evolved as a set of associated ideas and proposals, but in a relatively confined and stable network. The production and commercialization of machines using methods of quantum computation has greatly changed the territories of that network. In the process of commercialization, the theorization of quantum computing has been put into contact with particular domains of computing practice, and subject to the agencies of specific and unexpected materials. As the network of quantum computing is being grown by corporate actors, new strategies are used to make it of concern to broader areas of social, economic and political practice. This includes translating its technical systems into new kinds of representations that can create authority and credibility in business and legal domains. As these translations are negotiated and deployed, actors like 1QBit attempt to organize the emerging network into relationships that benefit them. These relationships create at least temporarily available resources for the network to become more real, as a new, viable, and accepted quantum computing system, by finding ways to connect it to established digital computing industry technologies and organizations. Rather than a paradox of quantum independence versus 
submersion in the existing digital landscape, this reflects the hybridization process of computational development. The extension of the quantum computing network complicates any simple divisions between quantum and digital paradigms, which only manage to maintain entirely separate modes of operation as abstractions. In order to propagate these hybrid objects and network, the ideas, skills, and boundaries of quantum mechanics/theoretical quantum computation are breached. A new mindset fosters the kinds of compromises that will be needed. This vision treats the success of quantum computing as a flexible target, linking the multiple computing paradigms drawn upon in its software frameworks to the framing of its research activity and business/legal descriptions. While the skillsets of the people involved with these very different areas of the network give them different ways of shaping and understanding the network, a working mindset allows the different layers of spokespersons to interact and adjust their shared vision for the network without disrupting its expansion. The new mindset provides a different and more flexible view to what a computer is and what it can be made to do, allowing work to continue in an uncertain technological terrain.

\subsection{Computation Forecasts}

New computing directions regularly emerge and are presented, and computation appears as a state of constant crises and revolution. However, quite outdated computing technologies are still used frequently alongside those supposed to lead to a revolution that never ever quite arrives (Mahoney 1988, Ende and 
Kemp 1999). Skepticism of the improvements offered by quantum computing has been part of its development well before any machines contended for the label (Landauer 1996). Whether any of the general, specific, or hybrid quantum systems proposed will actually deliver computation with exponentially different results from digital computation remains unknown. Yet, there is more certainty than ever around the expectation that at some point, in some configurations, in not too long, a quantum revolution will happen. This is especially notable given how little certainty there is about what future quantum computers will be made out of, what their most useful applications will be, and who will immediately benefit. This dissertation has highlighted key organizations and communities that are being developed toward, likely at the expense of other possible directions. Other directions are increasingly possible, as much work is being committed to make new actors care about the quantum. However, as the development process suggests, unexpected directions arise and the best intentions of business plans cannot entirely shape the capabilities of a computing network. The blind faith that can accompany new computer technologies and patterns of investment bubbles has been well critiqued (Cassidy 2002, Ceruzzi 2012). However, the process is messy for all those involved. Developing computation is risky because of this uncertainty and the limits of negotiation/translation imposed by the nonhuman.

What computers will be needed, and how that need can be generated in society, has historically been hard to predict. There are many mockable quotes about the inaccurate predictions of well-placed technological developers about the number, 
scale, or applications of digital computers that turned out to be exponentially wrong. Estimates that low-balled the number of computers that the world needed, often in the single digits per continent, didn't foresee the range of applications they would be put to work in and how these applications would change in conjunction with the perceived benefits of digital computation (Ende and Kemp 1999, Ensmenger 2010).

\subsection{Many-Worlds of Computing}

This dissertation has described how one quantum computing network has come into existence so far, and continues to integrate itself into a digital world. As it does, it builds upon existing technical and social foundations, to create new, novel, hybridized, and likely still evolving system of computing. How then to characterize this moment of quantum computation, when the blackbox is left so wide open? A temporary truce in negotiations? An unstable partnership? When first proposed by Callon (1986), translation is described as both a "process" and a "result". Computational networks are complex, shifting objects much better characterized as a process rather than a result, even though this breaks from the narratives of computer development provided by generations of product releases. Clearly the quantum computing network is still undergoing many transformations, and this helps stress the constant productive-mess of any computing network, which in technical descriptions can seem hyper-organized and orderly. Translation, as a framework for studying computation, can push forward this ongoing transformation, and bring to attention what actors are at work and what 
techniques they are deploying, rather than attempting to finalize and deconstruct the after-effects of a technical network.

Adiabatic quantum annealing, and its hybrid software developed for certain optimization problems, are just one implementation of a quantum computing network. As several chapters have mentioned, there are other ways to materialize and transform the theorizations of quantum mechanics into the workflows and organizational structures of a computing network. The breadth of computing technologies is likely to increase as more quantum systems multiply and find various ways to integrate with digital systems. The oracle-based quantum/digital partitioning approach described here, offers an intriguing example of how this process can occur, but it is unlikely to be the only way these connections are made, how computing responsibilities are negotiated, and how computational affordances develop. There will be many ways for quantum and digital actions to be intermingled. This makes it an exciting period to be studying computation. It suggests that the developments of quantum computing cannot be neatly understood, but will likely be constituted by an increasingly heterogeneous set of actors and technologies. These new quantum inclusions in computing networks can be examined to understand how computational agencies are distributed across new non-digital or hybrid actants, and to critique the social visions and kinds of work being prioritized in these distributions, without having to fall into the progress, revolution, or disruption-based accounts used by industry. These notions are mobilized as techniques to motivate the translation of quantum 
annealing in particular areas such as quantitative finance. 1QBit board member, Marcos Lopez de Prado, frames this as a new version of the digital divide within this privileged domain: "Digital-quants will be at an insurmountable disadvantage, when they are forced to compete with the coming generation of quantum-quants" (May 11, 2016). Rather than a fresh new paradigm cleanly separate from other computational methods, quantum computing networks are more heterogeneous than ever, mashing together concepts and techniques from the digital and analog, hybridizing computing frameworks and resources as necessary to enter new social domains.

AQC exists as an imperfect realization of the promises of quantum computing, and a process of shifting those promises to attainable engineering, computational and organizational goals. The durability of hybrid objects is not predictable. They can fall apart quickly, as AQC might in the face of another quantum network. Or AQC may be surprisingly robust compared to idealized theoretical networks that cannot be translated across a heterogeneous enough set of actors to be useful to the broader social world. Imperfect solutions can be surprisingly useful and long lasting, if they find the right social-technical roles; for example, the now several decades outdated original COBOL code is still used for $90 \%$ financial transactions and $75 \%$ of business data processing (Ensmenger 2010, 227). 


\subsection{Post-Processing ANT}

The translation process I have described involved some actors and spokespersons using many different modes to mobilize other actors/actants, drawing upon the authority derived from their representation. Actants are translated into data sets for benchmark graphs, descriptions for patent applications, and metaphoric landscapes. These transformations allow them to be brought out of the tightly constraining environments required for conditions such as superposition, and into environments such as business calls, presentations, or conferences. Many of these appear to be for the benefit of human readers or the requirements of organizations. Yet, similar textual-material translations are required to shift from analog magnetic field controllers to digital code. Each of these allow new readings of a translation to take place that enables new actions, but at the same time some readings can be wrong. Translation can fail or falter by not being faithful to the absent actors they claim to represent. The power of a translation/representation comes from its consensus across human and nonhuman actors, which perhaps overstates the obvious, that it needs to work. Mobilizations in this way become tests of representation for spokespersons. For example, whether a patent is granted helps extend qubits into a legal-economic dimension of existence. Each mobilization is a different way to evaluate spokespersons and the claimed reach of the network. Their transformations make entities such as qubits accessible to more actors and potentially subject to more non-technical and non-quantum actions. Each mode 
of mobilization provides another way to define the absent actors, to make them more real by increasing their social reach to meet their grasp.

As a whole, this dissertation presents a picture of how high tech is innovated, designed, and sold. It began with an interest of a localized controversy between academic physicists and commercial engineers that caught my attention. However, once in a position to follow the actors involved, controversy was not a framework that reflected much about the subject. It played a minor role, one that would have been easier to contain and present as a description. However, over time, the network emerged as much more banal construction activities, tests, and mobilizations. Daily problems were solved through ongoing translation attempts, which were less about controversy or closing down controversy, than they were about existing in a perpetual uncertainty. It built up new ideas and boundaries around hardware and software activities, and that congealed the network around an emergent mindset.

The example of quantum computing shows that many of the ways we have for understanding and describing computers are wrong, and may be increasingly misleading as computation is reordered. The technical outcomes of these systems cannot be isolated from their networks, and the broad area of negotiations conducted to construct them. Describing these as negotiations emphasizes the factor of nonhuman agency, implies that choices were made, and that these nonhumans/networks could have been connected/persuaded 
differently. This plays against the predictions and assertions of how the technology will continue to unfold. My description of these choices builds on socio-technical critiques of technology and technological development that describe technical change as a cumulative process of choices that configures "science, techniques, production routines, institutions, and engineering and social practices" in terms of a new technology (Ende and Kemp 1999). As quantum computing emerges, it brings with it a new grouping of knowledges, technologies, practices, and problems that appear as computational. An idea of how to solve physics simulations has morphed through many steps into proposing that their distant embodied cousins can solve issues like trading problems, and from there an unknown range of the otherwise intractable.

My deployment of ANT attempts to integrate some of its internal criticism from Latour $(2004,2005)$ and Law (2004), while not ignoring the value of its early focus on translation with socio-technical objects of study. This led me to focus my study around what made the quantum computing network seem increasingly real, even though its future is just as uncertain as ever. Uncertainty has been a recurring theme in the process and results of computational work described in these chapters. The negotiations that open up as a result of this uncertainty are not closed down, rather they spread that uncertainty throughout the resulting computing network, from qubit to corporate bulletin, using it to create room for new radical relationalities. 


\subsection{Socio-technical Innovation}

The growth of a quantum computing network provides a complex example of the process of high-technological development. It shows the many interactions that make computation possible, inside and outside of the computer itself. This should sensitize us to the socio-technical character of not only a technology's use, but its construction. Constructing computation is not a neutral process; it is shaped by the agencies of what is determined as negotiable. As an example, it shows how the many intentions behind technological development are often confounded, or redirected, by the agency of nonhumans and the process of translating ideas into specific materials and orders. The expectation for a technology often gets in the way of its actual development as it can deter necessary hybridizations. Technical development necessitates hybridizations of previous socio-technical orders with new actors, and is made possible when new ways to expand and shift relationality are found.

This makes it hard to account for what is behind a technology. It is easy to discount extremes of technological determinism or social constructivism, but the middle ground seems to leave us with a chaos of agencies, with no one to critique or hold accountable for the outcome of a technological innovation. Negotiations, however, are not haphazard processes. Technological innovation is an ongoing process of creating new heterogeneous relationships, which allows us to consider what technical possibility, of a finite amount, is pursued and negotiated into reality. 


\section{References}

Abramson, Bram Dov. (1998). Translating Nations: Actor-Network Theory in/and Canada. Canadian Review of Sociology, 35 (1): 1-19.

Agar, Jon. (2003).The Government Machine: A Revolutionary History of the Computer. Cambridge: MIT Press.

Akrich, Madeline. (1992). The De-scription of Technical Objects. In Bijker, W. and J. Law (Eds.), Shaping Technology / Building Society: Studies in Sociotechnical change, (205-224). Cambridge, MA: The MIT Press.

Anders, Janet, Saroosh Shabbir, Stefanie Hilt and Eric Lutz. (2010). Landauer's Principle in the Quantum Domain. In S.B Cooper, E. Kashefi, P. Panangaden. (Eds), Developments in Computational Models (13-18). EPTCS.

Appelbaum, Peter, Louis M. Friedler, Carlos E. Ortiz, and Edward F. Wolff. (2009). Internationalizing the University Mathematics Curriculum. Journal of Studies in International Education, 13(3): 365-381. 
Arns, Robert G. (1997). The High-Vacuum X-Ray Tube: Technological Change in Social Context Technology and Culture, 38(4): 852-890.

Baker, Karen S, Geoffrey C Bowker, and Helena Karasti. (2002). Designing An Infrastructure for Heterogeneity in Ecosystem Data, Collaborators and Organizations. [Proceedings]. 2002 Annual National Conference on Digital Government Research.

Bardini, Thierry and August T. Hovarth. (1995). The Social Construction of the Personal Computer User. Journal of Communication, (3): 40-66.

Barnes, Susan B. (2000). Bridging the Differences between Social Theory and Technological Invention in Human-Computer Interface Design. New Media \& Society, 2(3): 353-372.

Barocas, Solon, Sophie Hood, and Malte Ziewitz. (2013). Governing Algorithms: A Provocation Piece. SSRN, http://ssrn.com/abstract=2245322

Bell, Genevieve and Paul Dourish. (2006). Yesterday's Tomorrows: Notes on Ubiquitous Computing's Dominant Vision. Personal and Ubiquitous Computing, 11(2): 133-143. 
Bell, J.S. (2004).Speakable and Unspeakable in Quantum Mechanics: Collected Papers on Quantum Philosophy. Cambridge University Press.

Beniger, James. (1989). The Control Revolution: Technological and Economic Origins of the Information Society. Harvard University Press.

Bennett, Charles H. (1988). Notes on the History of Reversible Computation. IBM Journal of Research and Development, (32)1: 16-23.

Benson, Rodney. (2014). Challenging the "New Descriptivism": Restoring Explanation, Evaluation, and Theoretical Dialogue to Communication Research. [Proceedings] Qualitative Political Communication PreConference, International Communication Association, Seattle, May 22, 2014.

Berger, Peter L. and Thomas Luckmann. (1966). The Social Construction of Reality: A Treatise in the Sociology of Knowledge. New York: Penguin Books.

Berker, Thomas. (2006). The Politics of 'Actor Network Theory. Science, Technology, \& Innovation Studies: Special Issue 1: 61-79. 
Berman, Gennady P., Gary D. Doolan, Ronnie Maineri, and Vladimir Tsifrinovich. (1998). Introduction to Quantum Computers. World Scientific.

Bijker, W.E and Law, J.eds. (1992). Shaping Technology -- Building Society: Studies in Sociotechnical Change. MIT Press.

Bijker, W.E, Thomas Hughes, and Trevor Pinch. (1987). The Social Construction of Technological Systems: New Directions in the Sociology and History of Technology. MIT Press.

Bijker, W.E, Thomas Hughes, and Trevor Pinch. (2011). The Social Construction of Technological Systems: New Directions in the Sociology and History of Technology, Anniversary Edition. MIT Press.

Bijker, W.E. (1993). Do Not Despair: There Is Life after Constructivism. Science Technology Human Values, 18(1): 113-138.

Bijker, W.E. (2010). How Is Technology Made? That is the question! Cambridge Journal of Economics, 34: 63-76

Black, F. and Scholes, M. (1973). The Pricing of Options and Corporate Liabilities. Journal of Finance, 637-654. 
Black, Fischer. (1971). Toward a Fully Automated Stock Exchange, Part I. Financial Analysts Journal, 27( 6): 24-28.

Blok, Anders and Torben Elgaard Jensen. (2011). Bruno Latour: Hybrid thoughts in a hybrid world. Routledge.

Bloomfield, B. and Vurdubakis, T. (1999). The Outer Limits: Monsters, Actor Networks and the Writing of Displacement. Organization, 6: 625-48.

Bloor, David. (1976). Knowledge and Social Imagery. London: Routledge.

Bloor, David. (1999). Anti-Latour. Studies In History and Philosophy of Science, 30 (1): 81-112.

Bowker, Geoffrey C. (1994). Science on the Run. Cambridge: MIT Press.

Bowker, Geoffrey C., Star, Susan Leigh (2000). Sorting Things Out: Classification and Its Consequences. MIT Press.

Bozeman, Barry. Technology Transfer and Public Policy: A Review of Research and Theory. Research Policy, 29(4): 627-655. 
Brah, Avitar and Annie E. Combes. (Ed). (2000). Hybridity and its discontents: Politics, Science, Culture. New York: Routledge.

Buduma, Nikhil. (2016). Fundamentals of Deep Learning: Designing NextGeneration Artificial Intelligence Algorithms. New York: O'Reilly Media.

Callon, Michael and Fabian Muniesa. (2003). Economic Markets as Calculative Collective Devices. Reseaux, 21(22): 189-233.

Callon, Michel. (1981). Struggles and Negotiations to Define what is Problematic and what is not: The Socio-logic of Translation. In K. Knorr, R. Krohn \& R. Whitley. (Eds). The Social Process of Scientific Investigation. Reidel Publishing.

Callon, Michel. (1986). Some Elements of a Sociology of Translation: Domestication of the Scallops and the Fishermen of St Brieuc Bay. In Law, J. (Ed). Power, Action \& Belief. A New Sociology of Knowledge?, Routledge \& Kegan Paul: 196-229.

Callon, Michel. (1986b). The Sociology of an Actor-Network: The Case of the Electric Vehicle. In Callon, Michel Rip, Arie, Law, John (Eds.). Mapping the Dynamics of Science and Technology. (19-34). Palgrave Macmillan. 
Callon, Michel. and Fabian Muniesa. (2003). Economic Markets As Calculative Devices. Réseaux 21(122): 189-233.

Campbell-Kelly, Martin. (2007). The History of History of Software. IEEE Annals of the History of Computing, 29(4): 40-51.

Carey, James. (1989). Communication as Culture: Essays on Media and Society. Boston: Unwin Hyman.

Cassidy, John. (2002). Dot.con: How America Lost Its Mind And Money In The Internet Era. New York: Perennial.

Castells, Manuel. (1996). The Rise of the Network Society. Oxford: Blackwell.

Cerulo, Karen A. (2009). Nonhumans in Social Interaction. Annual Review of Sociology, 35: 531-552.

Ceruzzi, Paul E. (2003). A History of Modern Computing, 2nd ed. Cambridge: MIT Press.

Ceruzzi, Paul E. (2012). Computing: A Concise History. Cambridge: MIT Press. 
Chang, Pei-Chann, Chin-Yuan Fan, and Chen-Hao Liu. (2009). Integrating a Piecewise Linear Representation Method and a Neural Network Model for Stock Trading Points Prediction. IEEE Transactions on Systems, Man, and Cybernetics, 39(1): 80-92.

Chateauraynaud, F. (1991). Forces et failblesses de la novelle anthropologie des sciences. Critque, 529 (30): 459-78.

Chopra, Deepak. (2015). Quantum Healing: Exploring the Frontiers of Mind/Body Medicine. New York: Random House.

Christensen, Jens Frøslev and Peter Maskell. (2003). The Industrial Dynamics of the New Digital Economy. Edward Elgar Publishing

Clancy, Heather. (2012). IBM Cools Supercomputer with Hot Water. Greentech Pastures. June 19, 2012. http://www.zdnet.com/article/ibm-coolssupercomputer-with-hot-water/

Coleman, Gabriella. (2013). Coding Freedom: The Ethics and Aesthetics of Hacking. Princeton University Press.

Collins, H. (2011). Language and Practice. Social Studies of Science, 41 (2): 271-300. 
Collins, H. M., and Yearly, S. (1992). Epistemological Chicken. In A. Pickering (Ed.). Science as Practice and Culture, (301-326). Chicago: The University of Chicago Press.

Colouring Book of Patents. (2016). The National Archives of the United States. Available at: https://archivesaotus.files.wordpress.com /2016/02/ patents coloringbook.pdf

Conway, Steve, Earl C. Joseph, and Robert Sorensen. (2016). Quantum Computing in the Real World. IDC Technology Spotlight.

Cordella, Antonio amd Maha Shaikh. (2003). Actor Network Theory and After: What's New for IS Resarch? [Proceedings]. ECIS 2003. ECIS.

Cordella, Antonio and Maha Shaikh. (2006). From Epistemology to Ontology: Challenging the Constructed "truth" of ANT. London School of Economics and Political Science.

Couldry, Nick. (2008). Actor Network Theory and Media: Do they connect and on what terms? In Hepp, Andreas, Friedrich Krotz, Shaun Moores and Carsten Winter. (Eds.). Connectivity, Networks and Flows: 
Conceptualizing contemporary communications, (93-110). Hampton Press.

Cressman, Darryl. (2009). A Brief Overview of Actor-Network Theory: Punctualization, Heterogeneous Engineering \& Translation. Centre for Policy Research on Science and Technology. http://www.sfu.ca/sfublogsarchive/departments/cprost/uploads/2012/08/0901.pdf

David, Paul and Julie Bunn. (1988). The Economics of Gateway Technology and Network Evolution: Lessons From Electricity Supply History. Information Economics and Policy, (3)2: 165-202.

Davidson, Lloyd A. (2005). "The of Print: Digitization and Its ConsequenceRevolutionary Changes in Scholarly and Social Communication and in S cientific Research" International Journal of Toxicology January 2005 vol. 24 no. $125-34$

DeLanda, Manuel. (2006). A New Philosophy of Society: Assemblage Theory and Social Complexity. Bloomsbury Academic.

Deleuze, Gilles and Félix Guttari (1980). A Thousand Plateaus. A\&C Black. 
Dellarocas, Chrysanthos. (2003). The Digitization of Word of Mouth: Promise and Challenges of Online Feedback Mechanisms. Management Science (49)10: 1407-1424.

Denchev, Vasil S., Sergio Boixo, Sergei V. Isakov, Nan Ding, Ryan Babbush, Vadim Smelyanskiy, John Martinis, and Hartmut Neven. (2016). What is the Computational Value of Finite Range Tunneling? (v4) http://arxiv.org/abs/1512.02206

Dennet, Daniel C. (1995). Darwin's Dangerous Idea. New York: Simon and Schuster.

Deustch, David. (1985). Quantum Theory, the Church-Turing Principle and the Universal Quantum Computer. In Proceedings of the Royal Society of London, 400(18): 97-117.

Dillow, Clay. (2011). Lockheed Martin is Buying One of D-Wave's Brand New Quantum Computers. Popular Science, Technology. May 26, 2011.

Dolwick, Jim S. (2008). In Search of the Social: Steamboats, Square Wheels, Reindeer and Other Things. Journal of Maritime Archaeology. 3: 15-41. 
Doolin, Bill and Alan Lowe. (2002). To Reveal Is to Critique: Actor-network theory and critical information systems research. Journal of Information Technology 17( 2): 67-78.

Downey. Gary Lee. (1998). The Machine In Me. Routledge.

D-Wave Systems. (2015). Background: Quantum Computing Primer. [Report].

D-Wave Systems. (2015). Introduction to the D-Wave Quantum Hardware. [Report].

D-Wave Systems. (2015). The D-Wave 2x Quantum Computer: Technology Overview. [Brochure].

Dyer-Witheford, Nick. (1999). Cyber-Marx: Cycles and Circuits of Struggle In High Technology Capitalism. University of Illinois Press.

Dyson, George. (2012). Turing's Cathedral: The Origins of the Digital Universe. New York: Pantheon Books.

Edwards, Paul, S.J. Jackson, M.K. Chalmers, G.C. Bowker, C.L. Borgman, D, Ribes, M. Burton and S. Calvert. (2013). Knowledge Infrastructures: Intellectual Frameworks and Research Challenges. Ann Arbor: Deep Blue. 
Edwards, Paul, Steven J. Jackson, Geoffrey C. Bowker, and Cory P. Knobel. (2007). Understanding Infrastructure: Dynamics, Tensions, and Design. [Report]. History and Theory of Infrastructure: Lessons for New Scientific Cyberinfrastructures,NSF.

Edwards, Paul. (1988). The Closed World: Computers and the Politics of Discourse in Cold War America. MIT Press.

Effects of Information Technology on Financial Services Systems. (1984). [Report]. Office of Technology Assessment.

Emerson, Joseph. (2011). The Quantum Tamers. TVO. Department of Applied Math at the University of Waterloo. Available at: https://www.youtube.com/watch?v=J72pnLMlhKU

Ende, Jan van den and Rene Kemp. (1999). Technological Transformation in History: How The Computer Regime Grew Out Of Existing Computing Regimes. Research Policy 28(8): 833-85.

Ensmenger, Nathan L. (2012). The Computer Boys Take Over: Computers, Programmers, and the Politics of Technical Expertise. MIT Press. 
Eyring, Henry and Polanyi, Michael. (1931). On Simple Gas Reactions. Zeitschrift fur Physikalische Chemie B, 12:279-311.

Farias, Ignacio and Thomas Bender. (2009). Urban Assemblages: How ActorNetwork Theory Changes Urban Studies. Routledge.

Feynman, Richard. (1982). Simulating Physics with Computers. Journal of Theoretical Physics, 21: 467-488.

Feynman, Richard. (1985). Computer Heuristics Lecture. Available at: https://www.youtube.com/watch?v=EKWGGDXe5MA

Flinders, Karl. (2007). The Evolution of Stock Market Technology. Computer Weekly, Nov 02, 2007. ComputerWeekly.com.

Fogel, David B. (1998). Evolutionary Computation: The Fossil Record. WileyIEEE Press.

Freiberger, Paul and Michael Swaine. (1999). Fire in the Valley: The Making of the Personal Computer. McGraw-Hill .

Friedman, Andrew J. and Dominic S. Cornford. (1989). Computer Systems Development: History Organization and Implementation. New York: John Wiley \& Sons. 
Fuller, Matthew. (2008). Software Studies. MIT Press.

Gad, Christopher and Casper Bruun Jensen. (2010). On the Consequences of Post-ANT. Science, Technology \& Human Values, 35(1): 55-80.

Galison, Peter. 1996. Computer Simulations and the Trading Zone. In P. Galison and D. Stump. (Eds.). The Disunity of Science. Stanford: Stanford University Press.

Galloway, Alexander R. (2012). The Interface Effect. Polity Press.

Gillespie, Tarleton. (2014). The Relevance of Algorithms. In Gillespie, Tarleton Pablo J. Boczkowski and Kirsten A. Foot. (Eds.). Media Technologies: Essays on Communication, Materiality, and Society. (167-194). MIT Press.

Gillespie, Tarleton. (2016). Algorithm. In Ben Peters (Ed). Digital Keywords: A Vocabulary of Information Society and Culture, Princeton University Press.

Goffey, Andrew. (2008). Algorithm. In Software Studies: A Lexicon, Fuller, Matthew. (Ed). MIT Press. 
Graham, Margaret B.W. (2012). Intellectual Property and the Professionals. History and Technology, 28(1): 73-82.

Greimas, A.J. (1970). On Meaning: Selected Writing in Semiotic Theory. Minnesota University Press.

Grier, David. (1996). The ENIAC, the Verb "to program" and the Emergence of Digital Computers. IEEE Annals of the History of Computing, 18(1): 51-55.

Grossman, Lev. (2014). The Quantum Quest for a Revolutionary Computer. Time, Feb 6, 2014.

Guizzo, Erico. (2009). Loser: D-Wave Does Not Quantum Compute. IEEE Spectrum. Dec 31, 2009. http://spectrum.ieee.org/computing/ hardware/loser-dwave-does-not-quantum-compute

Hache, Emile and Bruno Latour. (2010). Morality or Moralism? Common Knowledge, 16(2): 311-330.

Hallyn, F. (Ed). (2013). Metaphor and Analogy in the Sciences. Springer Science \& Business Media. 
Halpern, Paul. (2015). Einstein's Dice and Schrodinger's Cat: How Two Great Minds Battled Quantum Randomness to Create a Unified Theory of Physics. Basic Books.

Hansmann, Ulrich H.E., and Luc T. Wille. (2002). Global Optimization by Energy Landscape Paving. Physics Review, 88: 6-11.

Hardie, lain, and Donald MacKenzie. (2006). Assembling an Economic Actor: The Agencement of a Hedge Fund. Presented at New Actors in a Financialised Economy and Implications for Varieties of Capitalism Institute of Commonwealth Studies. London, UK. May 11-12, 2006.

Hayles, Katherine N. (2004). Chaos Bound: Orderly Disorder in Contemporary Literature and Science. London: Cornell University Press.

Hayles, Katherine N. (2004). Print Is Flat, Code Is Deep: The Importance of Media-Specific Analysis. Poetics Today, 25(1): 67-90.

Hayles, Katherine N. (2005). My Mother Was A Computer: Digital Subjects and Literary Texts. The University of Chicago Press.

Hersch, Matthew. (2016). Spies and Shuttles: NASA's Secret Relationships with the DoD and CIA. Technology and Culture, (57)1: 275 - 276. 
Hill, Mark Donald, Norman Paul Jouppi and Gurindar Sohi. (2000). Readings in Computer Architecture. Gulf Publishing.

Hooft, Mark and Karen Swan. (2007). Ubiquitous Computing in Education: Invisible Technology, Visible Impact. Lawrence Erlbaum Associates.

Hughes, Thomas P. (1986). The Seamless Web: Technology, Science, Etcetera, Etcetera. Social Studies of Science, 16: 281-92.

Ifrah, Georges. (2000). The Universal History of Computing: From the Abacus to Quantum Computing. New York: John Wiley \& Sons.

Innis, Harold. (1950). Empire and Communications. Oxford: Clarendon Press.

Jallo, David and Daniel Budai. (2010). The Market Graph: A Study of its Characteristics, Structure \& Dynamics. [Thesis]. The Royal Institute of Technology.

Jones, Nicola. (2013). Computing: The Quantum Company. Nature: International Journal of Weekly Science. New Feature. June 19, 2013. 
Kadowaki, Tadashi and Hidetoshi Nishimori. (1998). Quantum Annealing in the Transverse Ising Model. Physics Review Letters, 58(5): 5355-5363.

Kaghan, William N. and Geoffrey C. Bowker. (2001). Out of Machine Age?: Complexity, Sociotechnical Systems and Actor Network Theory. Journal of Engineering and Technology Management,18: 253-269.

Kalai, Gil. (2006). How Quantum Computers Fail: Quantum Codes, Correlations in Physical Systems, and Noise Accumulation. Physics Review Letters, 95.

Katz, Michael L. and Carl Shapiro. (1998). Antitrust in the Digital Age. In Eisenach, Jeffrey and Lenard, Thomas M. (Eds.). Competition, Innovation and the Microsoft Monopoly: Antitrust in the Digital Marketplace Antitrust in Software Markets. Springer Science \& Business Media.

Katz, Raul L. and Pantelis Koutroumpis. (2010). Measuring Socio-Economic Digitization: A Paradigm Shift. (May 29, 2012). Available at SSRN: http://ssrn.com/abstract=2070035

Kaye, P., R. Laflamme and M. Mosca. (2007). An Introduction to Quantum Computing. Oxford University Press. 
Kelly, Ashley Rose and Kate Maddalena. (2015). Harnessing Agency for Efficacy: "Foldit" and Citizen Science. Poroi: An Interdisciplinary Journal of Rhetorical Analysis and Invention, Vol. 11(1): 1-20.

Keulartz, Jozef, Maartje Schermer, Michiel Korthals, and Tsjalling Swierstra. (2004). Ethics in Technological Culture: A Programmatic Proposal for a Pragmatist Approach. Science, Technology \& Human Values, 29(1): 3-29.

Kien, Grant. (2016). Actor-Network Theory. Serious Science, June 2, 2016. Available at: http://serious-science.org/actor-network-theory-5973

Kim, Byung-Keun. (2005). Internationalizing the Internet: The Co-evolution of Influence and Technology. Edward Elgar Publishing.

Kim, Kendall. (2007). Electronic and Algorithmic Trading Technology: The Complete Guide. Elsevier.

Kitchin, Rob. 2016. Thinking Critically about and Researching Algorithms. Information, Communication and Society, 20(1): 1-16.

Kittler, Friedrich. (1992). There is No Software. Stanford Literature Review, 9(1): 81-90. 
Klesse, Rochus and Sandra Frank. (2005). Quantum Error Correction in Spatially Correlated Quantum Noise. Submitted May 20, 2005. arxiv-quant-ph0505153

Kurzweil, Raymond. (1990). The Age of Intelligent Machines. MIT Press.

Lahlou, Saadi. (2010). Digitization and Transmission of Human Experience. Social Science Information, 49(3): 291-327.

Landauer, Rolf. (1996). The Physical Nature of Information. Physics Letters A 217: 188-193.

Lanzagorta, Marco and Jeffrey UhImann. (2010). The Complexity of Noise: A Philosophical Outlook on Quantum Error Correction. Morgan \& Claypool.

Latour, B. (1985). Give me a laboratory and I will raise the world. In KnorrCetina, K.D. and Mulkay, M.J. Science Observed: Perspectives on the Social Study of Science, London: Sage.

Latour, B. (1987). Science in action: How to follow scientists and engineers through society. Cambridge: Harvard University Press. 
Latour, B. (1996). On Actor-Network Theory: A Few Clarifications. Philosophia, 25(3): 369-381.

Latour, Bruno. (1988). The Pasteurization of France. Harvard University Press.

Latour, Bruno. (1993). We Have Never Been Modern. Harvard University Press.

Latour, Bruno. (1996b). Aramis, or the Love of Technology. Harvard University Press.

Latour, Bruno. (1996c). On Interobjectivity. Mind, Culture, and Activity, 3(4): 228245.

Latour, Bruno. (1999). On Recalling ANT. The Sociological Review, 47: 15-25.

Latour, Bruno. (2004). "An Attempt at a "Compositionist Manifesto" New Literary History, 41: 471-490.

Latour, Bruno. (2004). Why Has Critique Run out of Steam? From Matters of Fact to Matters of Concern. Critical Inquiry, 30: 225-248.

Latour, Bruno. (2004). The Politics of Nature: How to bring the sciences into democracy. Harvard University Press. 
Latour, Bruno. (2005). Reassembling the Social: An Introduction to ActorNetwork-Theory. Oxford University Press.

Latour, Bruno. (2005b). What is the Style of Matters of Concern? Spinoza Lectures. Presented by The Department of Philosophy of the University of Amsterdam. April 2005.

Latour, Bruno. (2010). Keynote: Reflections of an Actor-network theorist. International Journal of Communication, $5:$ 796-810.

Latour, Bruno. (2012). Gabriel Tarde and the End of the Social. In Joyce, Patrick. (Ed). The Social in Question: New Bearings. (117-32). London: Routledge.

Lavington, Simon. (1982). Early British Computers: The Story of Vintage Computers and the People Who Built Them. Manchester University Press.

Law, J. (1991). Theory and Narrative in the History of Technology: Response. Technology and Culture, 377-384.

Law, J. (1992). Notes on the Theory of the Actor-Network: Ordering, Strategy, and Heterogeneity. Systems Practice, 5 (4): 379-393. 
Law, J. (2003). Networks, Relations, Cyborgs: On the Social Study of Technology. Centre for Science Studies, Available at: www.comp.lancs.ac.uk/sociology/papers/Law-Networks-RelationsCyborgs.pdf

Law, J. (2004). After Method: Mess in Social Science Research. Routledge.

Law, J. and John Hassard. (Eds.). (1999). Actor Network Theory and After. Wiley- Blackwell.

Law, J. and John Hassard (eds). (1999). Actor Network Theory and After. WileyBlackwell.

Law, J. and John Urry. (2004). Enacting the Social. Economy and Society, 33(3): 390-410.

Law, J. and Michel Callon (1988). Engineering and Sociology in a Military Aircraft Project: A Network Analysis of Technical Change. Social Problems, (35): 284-297.

Law, J. and Vicky Singleton. (2007). Actor Network Theory and Material Semiotics. Available at: http://www.heterogeneities.net/publications/ Law2007ANTandMaterialSemiotics.pdf 
Law, J. (Ed). (1991), A Sociology of Monsters. Essays on Power, Technology and Domination, Routledge.

Leary, Robert H. (2000). Global Optimization on Funneling Landscapes. Journal of Global Optimization, 18(4): 367-383.

Lee.N. and S. Brown. (1994). Otherness and the Actor-Network: The Undiscovered Continent. American Behavioral Scientist, 37(6): 772-790.

Liang, Ko-Hsin. (1999). Combining Landscape Approximation and Local Search in Global Optimization. Evolutionary Computation, Proceedings of the 1999 Congress (Volume 2).

Linoff, Gordon S. and Michael J.A. Berry. (2011). Data Mining Techniques: For Marketing, Sales, and Customer Relationship Management. Wiley Publishing.

Lloyd, Seth. (2006). Programming the Universe: A Quantum Computer Scientist Takes on the Cosmos. Vintage Books.

Lloyd, Seth. (2013). The Universe as Quantum Computer. Available at: https://arxiv.org/abs/1312.4455 
Lopez de Prado, Marco. (2016). Q\&A on Financial Quantum Computing with Marcos Lopez de Prado. Quantum for Quants, May 11, 2016. http://www.quantumforquants.org/quantum-computing/qa-on-financialquantum-computing-with-marcos-lopez-de-prado/

Macey, David. (2000). The Penguin Dictionary of Critical Theory. Penguin Books.

Mahoney, Michael S. (1988). The History of Computing in the History of Technology . Annals History Computing, 10: 113-125.

Mahoney, Michael S. (2005). The Histories of Computing(s). Interdsicplinary Science. Reviews, (30) 2: 119-135.

Mahoney, Michael S. (2011). Histories of Computing. Harvard University Press.

Malerba, Franco, Richard Nelson, Luigi Orsenigo, and Sidney Winter. (2008). Public Policies and Changing Boundaries of Firms in a "History-Friendly" Model of the Co-Evolution of the Computer and Semiconductor Industries. Journal of Economic Behavior \& Organization, 67(2): 355-380.

Manovich, Lev. (2008). Software Takes Command. E-Pub. Version 11/20/2008. 
Markman, Gideon D., Donald S. Sigel, and Mike Wright. (2008). Research and Technology Commercialization. Journal of Management Studies, 45 (8): $1401-1423$.

Martin-Lopez, Enrique, Anthony Laing, Thomas Lawson, Roberto Alvarez, XiaoQi Zhou and Jeremy L On'Brien. (2012). Experimental Realization of Shor's quantum factoring algorithm using qubit recycling. Nature Photonics, 6: 773-776.

McCartney, Scott. (1999). Eniac: The Triumphs and Tragedies of the World's First Computer. Walker \& Company.

McGeoch, Catherine and Cong Wang. (2013). Experimental Evaluation of an Adiabatic Quantum System for Combinatorial Optimization. [Proceedings]. 2013 ACM Conference on Computing Frontiers. May 14, 2013.

McLean, Chris and John Hassard. (2004). Symmetrical Absence/Symmetrical Absurdity: Critical Notes on the Production of Actor-Network Accounts. Journal of Management Studies, 41(3): 493-519.

McLellan, David. (2007). Karl Marx Selected Writings, 2nd Ed. Oxford University Press. 
McLuhan. Marshall. (1964). Understanding Media: The Extensions of Man. New York: McGraw-Hill.

McMillan, Robert. (2014). The NSA is Building a Quantum Computer? We Already Knew That. Wired, Jan 3, 2014. Available at: http://www.wired.com/2014/01/hard_targets/

Meglicki, Zdzislaw. (2008). Quantum Computing with Magic. Cambridge: MIT Press.

Mermin, N. David. (2004). Could Feynman Have Said This?. Physics Today 57 (5): 10.

Merton, Robert. (1973). The Sociology of Science: Theoretical and Empirical Investigations. Chicago: University of Chicago Press.

Metroplis, N., J, Howlett and Gian-Carlo Rota. (Eds.). (1980). A History of Computing in the Twentieth Century: A Collection of Papers. New York: Academic Press.

Meyer, Keith. (2009). Extending and Simulating the Quantum Binomial Options Pricing Model. [Thesis]. The University of Manitoba. 
Miller, Rich. (2011). Facebook: 85 Degrees in the "Cold" Aisle. Data Center Knowledge, June 23, 2011.

http://www.datacenterknowledge.com/archives/2011/06/23/facebook-85degrees-in-the-cold-aisle/

Miller, Rich. (2012). Too Hot for Humans, But Google Servers Keep Humming. Data Center Knowledge. March 23, 2012.

http://www.datacenterknowledge.com/archives/2012/03/23/too-hot-forhumans-but-google-servers-keep-humming/

Miller, Rich. (2012b). How Google Cools Its Armada of Servers. Data Center Knowledge. October 17,2012.

http://www.datacenterknowledge.com/archives/2012/10/17/how-googlecools-its-armada-of-servers/

Mirowski, Philip and Robert Van Horn. (2005). The Contract Research Organization and the Commercialization of Scientific Research. Social Studies of Science, (35),4: 503-548.

Modis, T. and A. DeBecker. (1998). Innovation in the Computer Industry. Technological Forecasting and Social Change, 33: 267-278. 
Monea, Alexandre. (2016). Graph Force: Rhetorical Machines and the NArization of Knowlege. Computational Culture: A Journal of Software Studies. computationalculture.net/

Mosco, Vincent (2004) The Digital Sublime: Myth, Power, and Cyberspace. Cambridge, MA: MIT Press.

Muller, Christian. (2010). Black-box Landscapes: Characterization, Optimization, Sampling, and Application to Geometric Configuration Problems. [Diss]. University of Tubingen.

Mumford, Lewis. (1934). Technics and Civilization. Chicago University Press.

Murdoch, Jonathan. (1997). Inhuman/Nonhuman/Human: Actor-Network Theory and the prospects for a Nondualistic and Symmetrical Perspective on Nature and Society. Environment and Planning, 15: 731-56.

Murdoch, Jonathan. (2001). Ecologizing Sociology: Actor-Network Theory, Coconstruction and the Problem of Human Exemptionalism. Sociology, 35(1): 111-133.

Newell, Jenny. (2012). Old objects, New Media: Historical Collections, Digitization and Affect. Journal of Material Culture, (17) 3: 287-306. 
Nielsen, Michael A. and Isaac L. Chuang. (2011). Quantum Computation and Quantum Information 10th Ed. Cambridge University Press.

Nishimori, Hideoshi. (2014). Theory of Quantum Annealing. GoogleTech Talks. April 23, 2014. https://www.youtube.com/watch?v=OQ91L96YWCk

Niyato, Dusit, Sivadon Chaisiri, and Lee Bu Sung. (2009). Optimal Power Management for Server Farm to Support Green Computing. CCGRID '09 Proceedings of the 2009 9th IEEE/ACM International Symposium on Cluster Computing and the Grid: 84-91.

Noguchi, A. (2011). Quantum-state Tomography of a Single Nuclear Spin Qubit of an Optically Manipulated Ytterbium Atom. Physical Review, A: 84.

Novet, Jordan. (2013). Lockheed Martin Wants to Use a Quantum Computer to Develop Radar, Aircraft Systems. GIGAOM, Mar 22, 2013.

O'Connell, Brendan, Ciccotosto, Susan, and De Lange, Paul. (2014). Understanding the Application of Actor-Network Theory in the Process of Accounting Change. [Conference]. Critical Perspectives on Accounting Conference, 7-9 July 2014, Toronto, Canada. 
O'Regan, Gerard. (2008). A Brief History of Computing. Springer.

Orion's Belter. (2007, Feb 15). The Economist. Science \& Technology. http://www.economist.com/node/8697464

Park, Jay. (2010). New Cooling Strategies for Greater Data Center Energy Efficiency. Facebook. November 4, 2010. https://www.facebook.com/notes/facebook-engineering/new-coolingstrategies-for-greater-data-center-energy-efficiency/448717123919/

Parker, S. and Plenio M.B. (2000). Efficient factorization with a single pure qubit and $\log N$ mixed qubits. Physics Review Letters, 85: 3049-3052.

Parks, Lisa and Nicole Starosielski. (2015). Signal Traffic: Critical Studies of Media Infrastructures. University of Illinois Press.

Passoth, Jan-Hendrik and Nicholas J. Rowland. (2010). Integrating ActorNetwork Theory and State Theory. International Sociology, 25(6): 818841.

Pavicic, Mladen. (2006). Quantum Computation and Quantum Communication: Theory and Experiments. Springer. 
Pavlovic, Dusko and Catherine Meadows. (2011). Actor Network Procedures: Modeling Multi-factor Authentication, Device Pairing, Social Interactions. https://arxiv.org/pdf/1106.0706.pdf

Pereira, Anna, Juan P. Wachs, Kunwoo Park, and David Rempel. (2015). A User-Developed 3-D Hand Gesture Set for Human-Computer Interaction. Human Factors: The Journal of the Human Factors and Ergonomics Society, 57(4): 607-621.

Peters, John Durham. (2015). The Marvelous Clouds: Toward a Philosophy of Elemental Media. University Of Chicago Press.

Pikering, Andrew. (1995). The Mangle of Practice: Time, Agency, and Science. Chicago: The University of Chicago Press.

Pikering, Andrew. (2002). The Objects of Sociology: A Response to Breslau's "Sociology After Humanism". Sociological Theory, 18(2): 308-316.

Pinch, Trevor. (2010). On Making Infrastructure Visible: Putting the non-humans to rights. Cambridge Journal of Economics, 34(1): 77-89.

Politi, A., Mathews, J.C.F. and O'Brien, J.L. (2009). Shor's Quantum Factoring Algorithm on a Photonic Chip. Science, 325: 1221. 
Popper, Karl. (1963). Conjectures and Refutations. London: Routledge.

Pots, Lisa. (2008). Diagramming with Actor Network Theory: A Method for Modeling Holistic Experience. IEEE International Professional Communication Conference, July 13-16: 288-293.

Redmond, Kent C. and Thomas M. Smith. (1980). Project Whirlwind: History of a Pioneer Computer. Digital Press.

Ribes, David and Geoffrey C. Bowker. (2009). Between Meaning and Machine: Learning to Represent the Knowledge of Communities. Information and Organization, 19: 199-217.

Rich, Steven and Barton Gellman. (2014). NSA Seeks to Build Quantum Computer That Could Crack Most Types of Encryption. The Washington Post.com Jan 2, 2014.

Ripley, Colin, Geoffrey Thün, and Kathy Velikov. (2009). Matters of Concern. Journal of Architectural Education, 62(4): 6-14. 
Rønnow TF, Troyer M, Wang Z, Job J, Boixo S, Isakov SJ, Wecker D, Martinis JM: Defining and Detecting Quantum Speedup. Science, 19 June 2014. arXiv:1401.2910

Rosenberg, Gili, Poya Haghnegahdar, Phil Goddard, Peter Carr, Kesheng Wu and Marcos López de Prado. (2016). Solving the Optimal Trading Trajectory Problem Using a Quantum Annealer. IEEE Special Issue on Signal Processing and Machine Learning for Electronic Trading, 10(6): 1053-1060.

Rumming, Kristian. (2009). Following the Actors: mobilizing an actor-network theory methodology in geography. Australian Geographer, 40(4): 451-469.

Rutenbar, Rob A. (1989). Simulated Annealing Algorithms: An Overview. IEEE Circuits and Devices, 5(1): 19-26.

Sayes, Edwin. (2014). Actor-Network Theory and Methodology: Just What Does it Mean to Say That Nonhumans Have Agency? Social Studies of Science, 44(1): 134-149.

Schmidt, William H., Hsing Chi Wang \& Curtis C. McKnight. (2005). Curriculum Coherence: An Examination of US Mathematics and Science Content 
Standards From an International Perspective. Journal of Curriculum Studies, (37)5: 525-559.

Schoenfeld, Alan H. (2004). The Math Wars. Educational Policy, 18(1): 253-286.

Schwalm, Robert C. (1990). Human-Computer Interface Quality: Part 1-Process or Product? Proceedings of the Human Factors and Ergonomics Society, 34(4): 387-391.

Seaver, Nick. 2013. Knowing Algorithms. In Media in Transition 8. Cambridge, MA. Available at: http://nickseaver.net/s/seaverMiT8.pdf

Seidel, Robert. W. (1993). Evolving from Calculators to Computers. LANL Publications. https://library.lanl.gov/cgi-bin/getfile?9-24-93.html

Sergio Boixo, Troels F. Rønnow, Sergei V. Isakov, Zhihui Wang, David Wecker, Daniel A. Lidar, John M. Martinis, Matthias Troyer. (2013). Quantum annealing with more than one hundred qubits. Available at: http://arxiv.org/pdf/1212.1739v1.pdf

Seung Woo Shin, Graeme Smith, John A. Smolin, Umesh Vazirani. (2014). How 'Quantum' is the D-Wave Machine? http://arxiv.org/abs/1401.7087 
Shannon, C.E. (1948). A Mathematical Theory of Communication. The Bell System Technical Journal, (27): 379-423.

Sharkey, Jackie. (2016). Prime Minister Justin Trudeau Affirms $\$ 50 \mathrm{M}$ for Physics Think-Tank in Waterloo, Ont. CBCNews. Apr 15, 2016.

Sheller, Mimi and John Urry (2006). The New Mobilities Paradigm. Environment and Planning, 38: 207-226.

Sherwood, Mark H. \& Chuang, Isaac L. (2001). Experimental Realization of Shor's Quantum Factoring Algorithm Using Nuclear Magnetic Resonance. Nature, 414: 883-887.

Shiga, John (2006). Translations: Artifacts from an Actor-Network Perspective. Artifact, 1(1): 40-55.

Shirky, Clay. (2008). Here Comes Everybody: The Power of Organizing Without Organizations. London: Penguin Books.

Shor, Peter. (1994). Polynomial-time Algorithms for Prime Factorization and Discrete Logarithms on a Quantum Computer. Journal on Computing, 26(5): 1484-1509. 
Simonite, Tom. (2016). Moore's Law is Dead. Now What? Technology Review. https://www.technologyreview.com/s/601441/moores-law-is-dead-nowwhat/

Sismondo, Sergio. (2010). An Introduction to Science and Technology Studies. Oxford, Wiley-Blackwell.

Smith, Merritt Roe and Leo Marx. (1994). Does Technology Drive History: the Dilemma of Technological Determinism. Cambridge: MIT Press.

Sommerville, lan. (1999). Agency Versus Identity: Actor-network theory meets public relations. Corporate Communications, 4(1): 6-13.

Sommerville, lan. (2011). Software Engineering: 9th Edition. New York: AddisonWesley.

Spann, M.S., M. Adams and W. E. Souder. (2002). Measures of Technology Transfer Effectiveness: Key Dimensions and Differences in Their Use By Sponsors, Developers and Adopters. IEEE Transactions on Engineering Management, 42: 1.

Stadler, Felix. (2006). Manuel Castells : The theory of the Network Society. Cambridge: Polity Press. 
Star, S.L. and Ruhleder, K. (1996). The Ecology of Infrastructure: Problems in the implementation of large-scale information systems. Information Systems Research, 7(1), 111-134.

Star, Susan Leigh. (1999). The Ethnography of Infrastructure. American Behavioral Scientist, (43) 3: 377-391.

Star, Susan Leigh. (Ed). (1995). Ecologies of Knowledge: Work and Politics in Science and Technology. SUNY Press.

Starosielski, Nicole. (2015). The Undersea Network. Duke University Press.

Steane, A.M. (2003). A Quantum Computer Only Needs One Universe. Studies in History and Philosophy of Science Part B, 34 (3): 469-478.

Steiner, Christopher.( 2013). Automate This: How Algorithms Took Over Our Markets, Our Jobs, and the World. Portfolio.

Striphas, Ted. (2015). Algorithmic Culture. European Journal of Cultural Studies, 18(4-5): 395-412.

Sturman, S. (2006). On Black-boxing Gender: Some social questions for Bruno Latour. Social Epistemology, 20: 181-184. 
Swanson, Steven R. (2011). Google Sets Sail: Ocean-Based Server Farms and International Law. Connecticut Law Review, Vol. 43: 709.

Taminiau, T. H., J. Cramer, T. van der Sar, V. V. Dobrovitski \& R. Hanson. (2014). Universal Control and Error Correction in Multi-qubit Spin Registers in Diamond. Nature Nanotechnology, 9: 171-176.

Tatnall, Arthur and Anthony Gilding. (1999). Actor-Network Theory and Information Systems Research. 10th Australasian Conference on information systems (ACIS), Victoria University of Wellington, Wellington, New Zealand.

Tatnall, Arthur. (2005). Technological Change in Small Organisations: An innovation translation perspective. International Journal of knowledge, Culture and Change Management, 4: 755-761.

Tatnall, Arthur. (Ed). (2010). Actor-network Theory and Technology Innovation: Advancements and New Concepts. Information Science Publishing.

Thomas, Keir. (2011). Lockheed Martin Bets Big on Quantum Computing. PCWorld. May 28, 2011. 
Tilson, Arthur. and K. Lyytinen. (2005, June 1-3). An Actor-Network Study of $3 G$ Standards Making and Adoption in the US Wireless Industry - A Case Study of Wireless Operator Standardization Strategies. [Conference Paper]. Hong Kong Mobility Roundtable, Hong Kong.

Troyer, Matthias. (2014). Benchmarking the D-Wave Two. Bulletin of the American Physical Society. 59(1).

Turing, Alan. (1936). On Computable Numbers, With an Application to the Entscheidungs Problem. London Math Society, 42(1): 230-265.

Turner, Fred. (2005). Actor-networking the News. Social Epistemol. 19(4): $321-$ 24.

Turner, Fred. (2006). From Counterculture to Cyberculture. University of Chicago Press.

Urry, John. (2004). The System of Automobility. Theory, Culture \& Society, (21)4: 25-39.

Vandersypen, Lieven M. K., Steffen, Matthias; Breyta, Gregory, Yannoni, Costantino S. Experimental Realization of Shor's Quantum Factoring Algorithm Using Nuclear Magnetic Resonance. Nature, 414: 883-887. 
Vannini, P. (Ed). (2009). Material Culture and Technology in Everyday Life : Ethnographic Approaches. New York: Peter Lang Publishing.

Venturelli, Davide, Galo Rojo, and Dominic Marchand. Quantum Annealing Implementation of Job Scheduling Problems. Available at: https://arxiv.org/abs/1506.08479

Venturini, Tommaso. (2010). Diving in Magma: How to Explore Controversies with Actor-network Theory. Public Understanding of Science, 19(3): 258273.

Vinci, Walter, Tameem Albash, Anurag Mishra, Paul A. Warburton, and Daniel A. Lidar (2014). Distinguishing Classical and Quantum Models for the DWave Device. Physics Review A 91.

von Neumann, John. (1958) The Computer and the Brain: Perspectives on Human and Artificial Intelligence. Yale University.

Wark, MacKenzie. (2004). A Hacker Manifesto. Harvard University Press.

Watson, Gavan. (2007). Actor Network Theory, After-ANT \& Enactment: Implications for Method. Available at: http://www.gavan.ca/wpcontent/uploads/2007/01/ANT_comp.pdf 
Weinberg D. (1997). The Social Construction of Non-human Agency: The Case of Mental Disorder. Social Problems, 44(2): 217-34.

Whatmore, S. (2002). Hybrid Geographies: Natures, Cultures, Spaces. Sage, London.

William-Jones, Bryn and Janice E. Graham. (2003). Actor-Network Theory: A tool to support ethical analysis of commercial genetic testing. New Genetics and Society, 22(3): 271-296.

Williams, Michael R. (1997). A History of Computing Technology, 2nd Edition. IEEE Computer Society Press.

Winner, L. (1980). Do Artifacts Have Politics? Daedalus 109(1): 121-136.

Wolfram, Steven. (2002). A New Kind of Science. Wolfram Research.

Woolgar, Steve and Geoff Cooper. (1999). Do Artifacts Have Ambivalence? Moses' Bridges, Winner's Bridges and Other Urban Legends in S\&TS. Social Studies of Science, 29(3): 433-449. 
Wu, Yanquing, Vasili Perebeinos, Yu-ming Lin, Tony Low, Fengnian Xia, and Phaedon Avouris. (2012). Quantum Behavior of Graphene Transistors near the Scaling Limit. IBM Thomas J. Watson Research Center. Nano Letters.12: 1417-1423.

Wyatt, Sally. (2008). Technological Determinism is Dead; Long Live Technological Determinism. The Handbook of Science and Technology Studies, 10(1): 165-180.

Yanqing Wu, Vasili Perebeinos, Yu-ming Lin, Tony Low, Fengnian Xia, and Phaedon Avouris. Quantum Behavior of Graphene Transistors Near the Scaling Limit. Nano Lett, 12(3): 1417-1423.

Yates, Joanne. (2005). Structuring the Information Age: Life Insurance and Technology in the Twentieth Century. John Hopkins Press.

Young, Nathan. (2006). Distance as a Hybrid Actor. Journal of Rural Studies, 22(3): 253-266.

Zaribafiyan, Arman and Jaspreet Oberoi. (2015). Asset Allocation in Cognitive Radio Scenarios. 1QBit. Available at: http://1qbit.com/wpcontent/uploads/2016/05/1QBit-White-Paper-\%E2\%80\%93-CognitiveRadio-Project.pdf 
Zhengbing, Brian, Fabian Chudak, William G. Macready, Geordie Rose. (2010). The Ising Model: Teaching An Old Problem New Tricks. D-Wave Systems.

Ziewitz, Malte. (2016). Governing Algorithms: Myth, Mess, and Methods. Science, Technology \& Human Values, 41(1): 3-16.

Ziewitz, Malte. (2011). How to Think About an Algorithm? Notes from a not quite random walk". [Conference]. Symposium on Knowledge Machines between Freedom and Control, 29 September. Available at: http://zwtz.org/files/ziewitz_algorithm.pdf

Zimmermann, Severin, Ingmar Meiger, Manish K. Tiwari, Stephan Paredes, and Bruno Michel. (2012). Aquasar: A Hot Water Cooled Data Center with Direct Energy Reuse. Energy, 43: 237-245.

\section{Websites}

1QBit. http://1qbit.com/

D-Wave Systems. http://www.dwavesys.com/

Schtel-Optimized. http://www.scottaaronson.com/blog/

Hack the Multiverse. https://dwave.wordpress.com/

Cornel University Library. arXiv. http://arxiv.org/

Mitacs. http://www.mitacs.ca/e 


\section{Appendix A}

A.1 Initial 1QBit Core Reading

Physics Reference:

Choi, Vicky. (2010). Adiabatic Quantum Algorithms for the NP-Complete Maximum-Weight Independent Set, Exact Cover and 3SAT Problems. arXiv. Available at: http://arxiv.org/abs/1004.2226

Johnson, M. W. , M. H. S. Amin, S. Gildert, T. Lanting, F. Hamze, N. Dickson, R. Harris, A. J. Berkley, J. Johansson, P. Bunyk, E. M. Chapple, C. Enderud, J. P. Hilton, K. Karimi, E. Ladizinsky, N. Ladizinsky, T. Oh, I. Perminov, C. Rich, M. C. Thom, E. Tolkacheva, C. J. S. Truncik, S. Uchaikin, J. Wang, B. Wilson and G. Rose. Quantum annealing with manufactured spins. doi:10.1038/nature10012

Rose, Geordie and William G. Macready. (2007). An Introduction to Quantum Annealing. D-Wave Systems.

Santoro , Giuseppe E., Roman Martoñák, Erio Tosatti, and Roberto Car. (2008). Theory of Quantum Annealing of an Ising Spin Glass. http://arxiv.org/abs/cond-mat/0205280v1 
Mathematical Reference:

Barry A. Cipra, "An Introduction to the Ising Model". The American Mathematical Monthly, 94(10): 937-959.

Edward Farhi, Jeffrey Goldstone, Sam Gutmann, Joshua Lapan, Andrew Lundgren, Daniel Preda (2001). A Quantum Adiabatic Evolution Algorithm Applied to Random Instances of an NP-Complete Problem. http://arxiv.org/abs/quant-ph/0104129v1

Gabriel Tavares (2008). New Algorithms for Quadratic Unconstrained Binary Optimization (QUBO) with Applications in Engineering and Social Sciences. [Diss]. Rutgers University.

Horowitz, Ellis; Sahni, Sartaj (1974). Computing Partitions with Applications to the Knapsack Problem. Journal of the Association for Computing Machinery, 21: 277-292.

Kindermann, Ross, J. Laurie Snell, (1980). Markov Random Fields and Their Applications. American Mathematic Society .

Martello, Silvano; Toth, Paolo. (Ed). (1990). 4 Subset-sum problem. Knapsack Problems: Algorithms and Computer Interpretations. Wiley-Interscience. 
Zhengbing Bian, Fabian Chudak, William G. Macready, Geordie Rose, (2010). The Ising model: teaching an old problem new tricks. D-Wave Systems.

Financial Reference:

Omar Rifki, Hirotaka Ono. (2012).A Survey of Computational Approaches to Portfolio Optimization by Genetic Algorithms. 18th International Conference Computing. Department of Economic Engineering. Kyushu University. http://catalog.lib.kyushu-u.ac.jp/handle/2324/25317/CEF2012-167.pdf

Bailey, David H. and Lopez de Prado, Marcos. (2013). An Open-Source Implementation of the Critical-Line Algorithm for Portfolio Optimization Algorithms, 6(1): 169-196, 2013. Available at SSRN: http://ssrn.com/abstract=2197616

Crama Y., M. Schyns. (2003). Simulated Annealing for Complex Portfolio Selection Problems. European Journal of Operational Research, 150: $546-571$.

Forsyth, Peter. (2013). An Introduction to Computational Finance Without Agonizing Pain. May 16, 2013. Cheriton School of Computer Science, University of Waterloo. https://cs.uwaterloo.ca/ paforsyt/agon.pdf 
Hull, John C. (2009). Options, Futures, and other Derivatives. 7th edition. Toronto: Pearson Hall.

Phelim Boyle, Mark Broadie, Paul Glasserman. Monte Carlo methods for security Pricing. Journal of Economic Dynamics and Control, 21: 1267-1321.

Keith Meyer (2009). Extending and Simulating the Quantum Binomial Options Pricing Model. [Thesis]. The Department of Computer Science, The University of Manitoba.

D-Wave Tutorials:

D-Wave Systems. (2009). Adiabatic Quantum Computing with the D-Wave One.

D-Wave Systems. (2011). Programming with QUBOs. 
A.2 1QBit-Mitacs Proposals. Available at: https://www.mitacs.ca/en Applied Research in Quantum-based Solutions for Optimization Problems

Applied Research in Quantum-based Solutions for Optimization Problems

Fundamental Research in Quantum Annealing Algorithms for Optimization Problems

Investigating NP-Hard Optimization Problems with a Quadratic Binary Solver Investigating QUBO-Compatible Algorithms for Trading and Scheduling Optimization Problems

Investigating the Implementation of Machine Learning Algorithms on Adiabatic Quantum Solvers

Programming Techniques for QUBO Compatible Processors

Programming Techniques for QUBO Compatible Processors II

Programming Techniques for QUBO Compatible Processors III

The Communicative Challenges and Implications of Quantum Computing 
The Communicative Challenges and Implications of Quantum Computing Renewal

Translating a Quantum Computing Actor-Network Through Controversy 